\title{
The impact of model-lead-test coaching on parents' implementation of reinforcement, prompting, and fading with their children with autism spectrum disorder
}

Liyu Chen

Follow this and additional works at: https://researchrepository.wvu.edu/etd

\section{Recommended Citation}

Chen, Liyu, "The impact of model-lead-test coaching on parents' implementation of reinforcement, prompting, and fading with their children with autism spectrum disorder" (2015). Graduate Theses, Dissertations, and Problem Reports. 5347.

https://researchrepository.wvu.edu/etd/5347

This Dissertation is protected by copyright and/or related rights. It has been brought to you by the The Research Repository @ WVU with permission from the rights-holder(s). You are free to use this Dissertation in any way that is permitted by the copyright and related rights legislation that applies to your use. For other uses you must obtain permission from the rights-holder(s) directly, unless additional rights are indicated by a Creative Commons license in the record and/ or on the work itself. This Dissertation has been accepted for inclusion in WVU Graduate Theses, Dissertations, and Problem Reports collection by an authorized administrator of The Research Repository @ WVU.

For more information, please contact researchrepository@mail.wvu.edu. 
THE IMPACT OF MODEL-LEAD-TEST COACHING ON PARENTS'

IMPLEMENTATION OF REINFORCEMENT, PROMPTING, AND FADING WITH

THEIR CHILDREN WITH AUTISM SPECTRUM DISORDER

Liyu Chen

Dissertation submitted to the College of Education and Human Services,

West Virginia University, in partial fulfillment of the requirements

for the degree of

Doctor of Education

In

Educational Psychology

Daniel Hursh, Ph.D., Chair

Reagan Curtis, Ph.D.

Bobbie Warash, Ed.D.

Jose Martinez-Diaz, Ph.D.

Michael Mayton, Ph.D.

Department of Educational Psychology

Morgantown, West Virginia

2014

Keywords: model-lead-test, competent learner model, reinforcement, prompting, fading. 


\begin{abstract}
The Impact of Model-Lead-Test Coaching on Parents' Implementation of Reinforcement, Prompting, and Fading with Their Children with Autism Spectrum Disorder
\end{abstract}

\title{
Liyu Chen
}

Parents play an essential role in furthering the development of their children with special needs. They are being trained to be co-therapists for their own children. The goal is to improve the ways they interact with their children in order to create improvements in their children's everyday functioning. If the proper teaching strategies are consistently applied, a learner can significantly improve his/her performance of various life skills, including communication, self-care, social skills, along with other skill sets. Because adults' learning processes differ substantially from children's, it will be critical to utilize the "coaching" method that employs a Model-Lead-Test (MLT) approach to effectively train parents of children with autism. The primary purpose of this study is to evaluate the impact of modellead-test coaching on parents' use of prompting, fading, and reinforcement with their children with Autism Spectrum Disorder (ASD). The secondary purpose is to assess whether there are improvements in the children's talker, participator, and problem solver repertoires associated with their parents' use of these behavior change processes.

Using a multiple baseline across behavior design for each parent-child dyad, data are collected on parents' proper use of reinforcement, prompting, and fading as well as their children's talker, participator, and problem solver repertoire development. Research phases include baseline, parent training I (Oral Lecture), parent training II (Model-Lead-Test), and maintenance.

The resulting data from this study indicate that the Model-Lead-Test approach to parent implementation of core ABA strategies has a greater impact than merely using an Oral Lecture Discussion approach to parent training. To summarize, the data from all three participants showed an increase in the proper implementation of reinforcement, prompting and fading procedures especially through MLT training. The child participants also showed an increase in their talker, participator and problem solver repertoires. And finally, interpretation of the data is presented along with possible future guidelines for research. 
Table of Contents

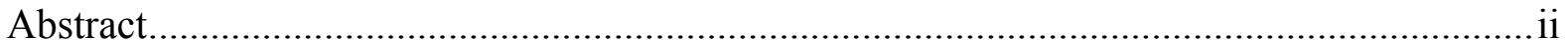

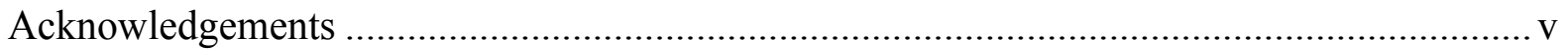

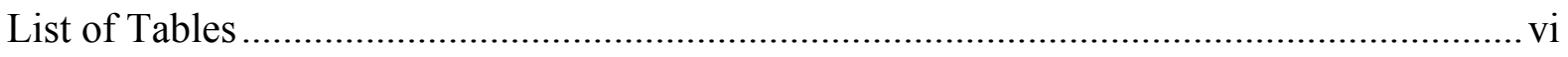

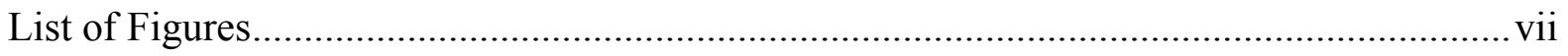

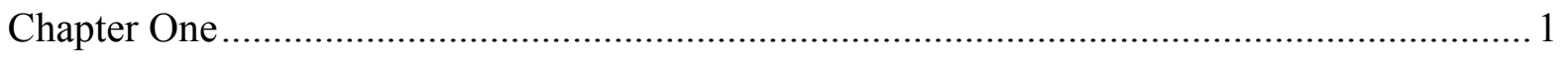

Introduction and Review of the Literature ................................................................... 1

Challenges Associated with Autism ...................................................................... 1

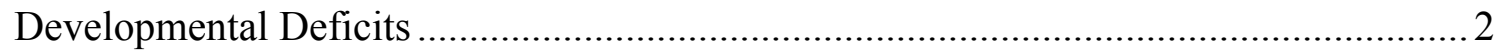

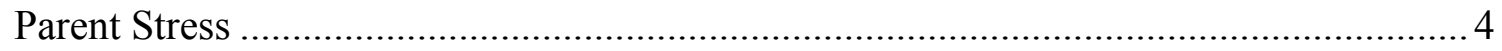

Core Concepts of Applied Behavior Analysis.......................................................... 5

Parent Participation in the Training of Their Children.................................................... 8

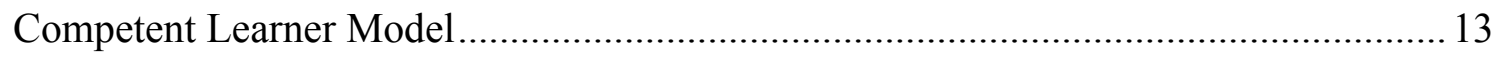

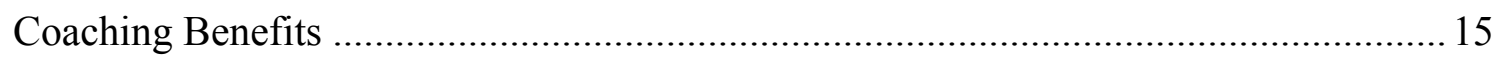

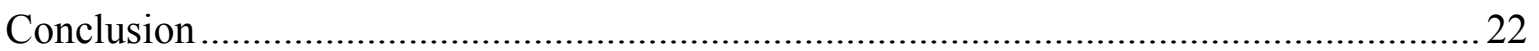

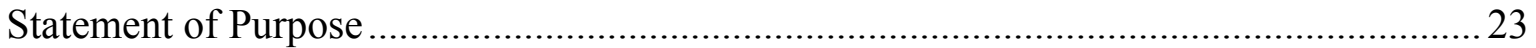

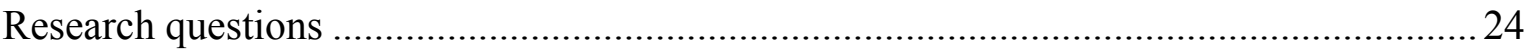

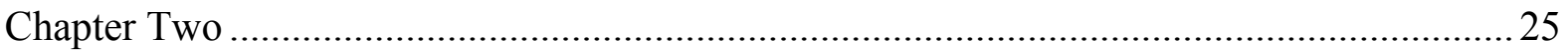

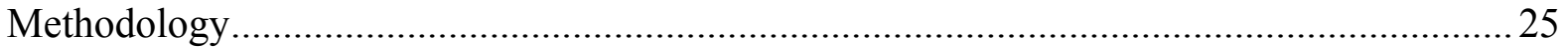

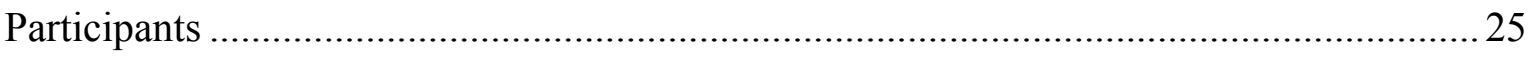

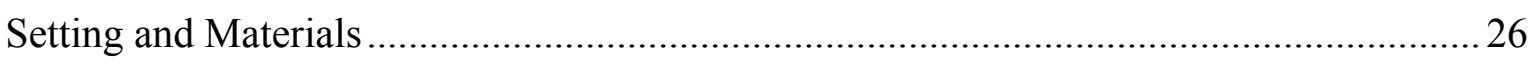

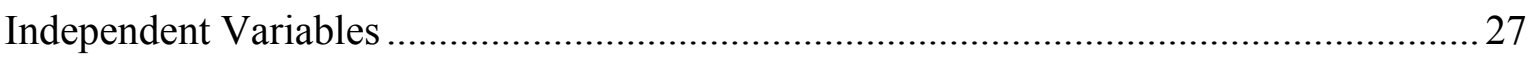

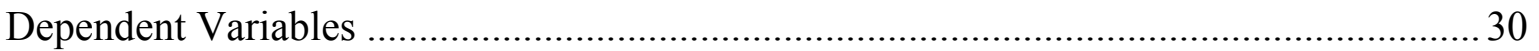

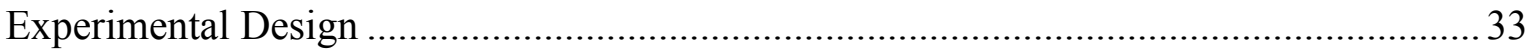

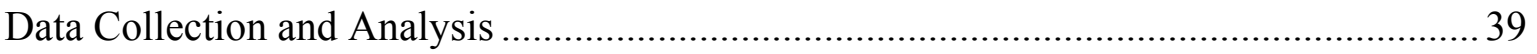

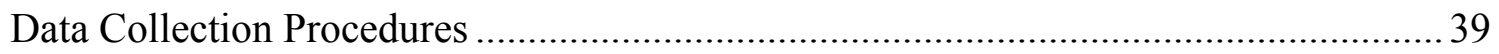

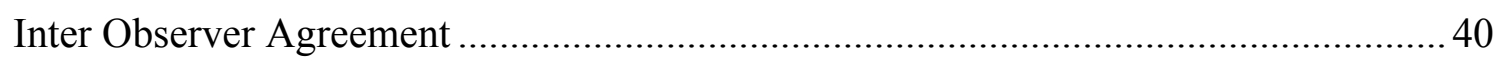

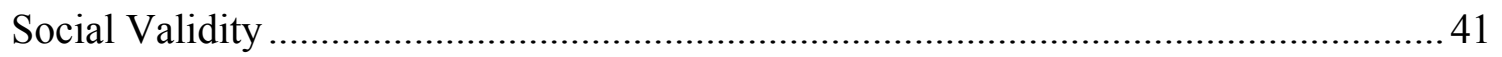

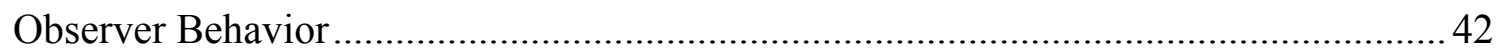

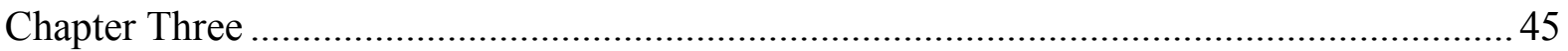

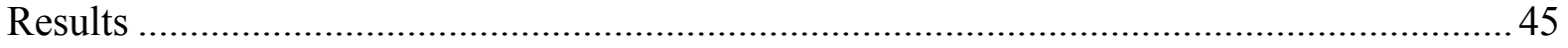

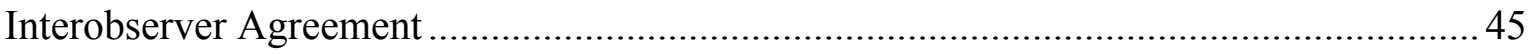

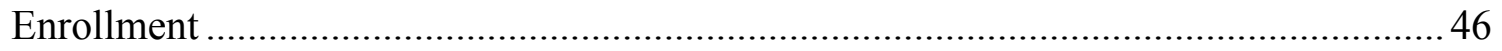

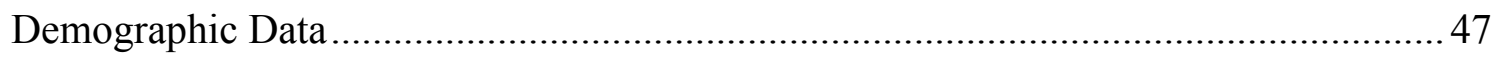

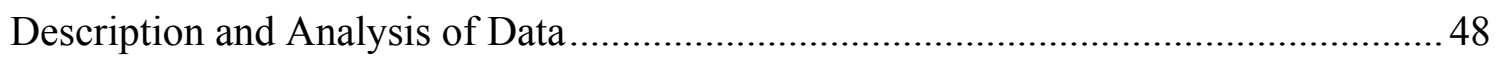




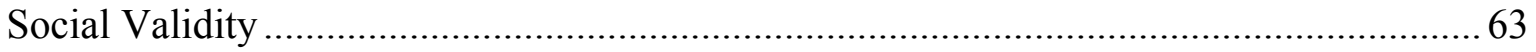

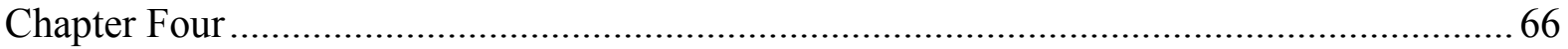

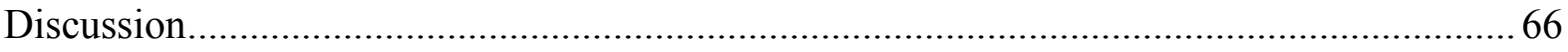

Is Parent Training Using a Model-Lead-Test Procedure Effective in Increasing Parents' Appropriate Use of Reinforcement, Prompting, and Fading Techniques?.

Do the Learner's Talker, Participator, and Problem Solver Repertoires Improve for Learners with Autism Spectrum Disorder as Their Parents' Appropriate Use of Reinforcement, Prompting, and Fading Techniques Increase? 70

Limitations and Discussions for Future Research ....................................................... 71

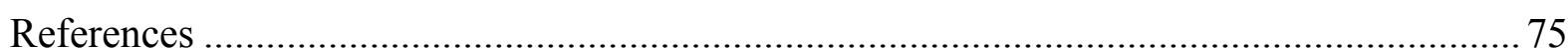




\section{Acknowledgement}

I would like to acknowledge my advisor, Dr. Daniel Hursh, for your consistent support, patience, and encouragement on this long journey throughout my doctoral program. Thank you for all of the great opportunities you have created for me throughout my academic learning as well as being inspirational in my personal life. In my mind, you are not only my advisor and mentor but also a heartfelt father figure to me in the United States. I have immense respect for you and greatly value your life mentorship. Without your unconditional support, I could not have achieved my goals and be who I am today.

Thank you to my committee for helping me through the doctoral program and dissertation process. Dr. Martinez for your support and resourcefulness at anytime I needed extra assistance and for enlightening and enticing me into the field of ABA; Dr. Warash for your teaching when I was working in the daycare center and for your inspirational input and positive feedback for my research; Dr. Curtis for the great training in the research class as well as your suggestions and extensive editing throughout my dissertation; Dr. Walls for your instructions in Psychological Foundations of Learning, your thorough commentary in my prospectus and feedback in my references; Dr. Mayton for joining my committee when Dr. Walls retired and coaching me with tips on graphing.

I want to thank several colleagues who have also provided their support during these many years. I want to thank my employer, Vicci Tucci, for giving me a great opportunity in my current position to learn and grow in the ABA field; Ms. Ruth, for supporting me as a family member and always making yourself available when I needed you most; David Nabhan, for your support and encouragement, Josh Kayne for always sharing invaluable information and for knowing exactly when I needed a hug.

I also want to thanks for my parents for being so proud of what I am doing in my field; my sister and brother for taking care of our parents when I am in the USA; my best friend, Ming, for endless encouragement. And finally, to Jacob Jones, for your consistent help, ability to show me the beauty in life around me, and bringing hope and positive energy into my life. You have revitalized me and helped me to find my true self once again.

Last, thank you to all of my participants who have been involved in my study. 
List of Table

\section{Table 1. Definitions for Reinforcement, Prompting and Fading}

Table 2. Process, Contents, and Activities for each OLD Presentation Session

Table 3. Operational Definitions of Parent Behavior

Table 4. Operational Definitions of Child Behavior

Table 5. The Possibilities of Study Design

Table 6. Demographic Data for the Three Parent Participants

Table 7. Demographic data for the Three Child Participants

Table 8. Means, Ranges, and Levels of Mark's Percent of Implementing Proper Reinforcement, Prompting, \& Fading

Table 9. Means, Ranges, and Levels of Brandy's Percent of Implementing Proper Reinforcement, Prompting, \& Fading

Table 10. Means, Ranges, and Levels of Jenny's Percent of Implementing Proper Reinforcement, Prompting, \& Fading

Table 11. Means, Ranges of Children's Percentage of Talker Repertoire

Table 12. Means, Ranges of Children's Percentage of Participator Repertoire

Table 13. Means, Ranges of Children's Percentage of Problem Solver Repertoire

Table 14. Social Validity Questionnaire for Parents in OLD

Table 15. Social Validity Questionnaire for Parents in MLT 
List of Figures

Figure 1. Mark's Percentage of Proper Implementation of Reinforcement, Prompting, and Fading

Figure 2. Brandy's Percentage of Proper Implementation of Reinforcement, Prompting, and Fading

Figure 3. Jenny's Percentage of Proper Implementation of Reinforcement, Prompting, and Fading

Figure 4. The Means for Child Participants' Talker Repertoires

Figure 5. The Means for Child Participants' Participator Repertoires

Figure 6. The Means for Child Participants' Problem Solver Repertoire 


\section{Chapter One}

\section{Introduction and Review of the Literature}

\section{Challenges Associated with Autism}

The challenges associated with rearing a child with autism spectrum disorders have been well documented over the past several decades (Davis \& Carter, 2008). There has been a substantial increase in the number of children diagnosed with autism as revealed by schools, clinicians, and service agencies worldwide (Bax, 1994; Cox, Klein \& Charman, 1999; Department of Developmental Services, 1999; Croen, Grether, Hoogstrate, \& Selvin, 2002). The Centers for Disease Control and Prevention (2014) have estimated that ASD occurs in 1 in 68 children age 8 in the United States. Boys ( 1 in 42$)$ are almost five times more likely to have an ASD than girls (1 in 189). Researchers have claimed that the growing rate of autism might be caused by a different standard for diagnosis of autism because there was no agreement about the classification system and diagnostic devices in the previous decades and this may account for the observed increase in autism prevalence (Fombonne, 2005). Regardless, the estimated incidence of about 1 in 68 children with ASD makes ASD a significant developmental delay (Croen, Grether, Hoogstrate, \& Selvin, 2002; Malfa, Lassi, Bertelli, Placidi, \& Salvini, 2004). CDC Director Dr. Julie Gerberding stated, "Our estimates are becoming better and more consistent, though we cannot yet tell if there is a true increase in ASDs or if the changes are the result of our better studies. We do know, however, that these disorders are affecting too many children" (cited by Bradley, 2007). Heward (2006) wrote, "autism is one of the most frightening, exhausting, and heartbreaking experiences for the parents and families of children with the condition".

Because of the challenge from the unknown reasons for and increasing rate of individuals with Autism Spectrum Disorder in recent decades, Autism Speaks estimated that $\$ 137$ billion is spent in providing care for all people with ASD each year and between 1.4 
and 2.3 million is spent for individuals with autism in their lifetime (Autism Speaks, 2012). Therefore, it is important for us to provide early intervention services that help individuals with autism to achieve the best habilitation possible. Parental involvement in their children's treatment is of paramount importance.

\section{Developmental Deficits}

Autism Spectrum Disorder (ASD) is a type of developmental illness in the brain. ASD manifests as a range of complex neurodevelopment and genetic disorders characterized by a spectrum of symptoms divided into three categories. According to guidelines listed in the Diagnostic and Statistical Manual of Mental Disorders, Fifth Edition - Text Revision (DSM-V-TR, 2014), the symptoms of autism spectrum disorder vary significantly in character and severity from one child to the next. It occurs in all socioeconomic and ethnic groups, and impacts every age group. In general, the symptoms fall into three areas: social impairments, communication difficulties, and restricted, repetitive, and stereotyped patterns of behavior. Impairment in social interaction includes having difficulties engaging in multiple nonverbal behaviors such as eye contact, facial expression, body posture, and gestures to regulate social interaction. Impairment in communication includes delay in, or total lack of, the development of spoken language, stereotyped and repetitive use of language, or idiosyncratic language. Restricted, repetitive, and stereotyped patterns of behavior are evidenced as preoccupations with one or more stereotyped and restricted patterns of interest.

Very often children with characteristics of an ASD may also be diagnosed with Fragile X syndrome, tuberous sclerosis, Tourette syndrome, epileptic seizures, learning disabilities, and attention deficit disorder. In other words, children with a previous ASD classification and co-occurring psychiatric or neurologic condition have had these additional conditions discovered when they were older (Levy et al., 2010). In fact, there is high percentage, about 20 to 30 percent, of children with autism who develop a seizure disorder 
and some of these children with autism may not experience seizures until their adolescence. Gurney et al. (2006) report that children with an autism classification were significantly more likely to have a variety of medical and psychiatric conditions, frequent physician visits for preventive care, non-emergency and hospital emergency care and higher medication usage than children without autism. Also, Anxiety, Depression, Attention Deficit Hyperactivity Disorder, Obsessive-Compulsive Disorder, Psychotic Disorders, Bipolar Disorder, and Oppositional Defiant Disorder frequently co-occur in individuals with autism spectrum disorders and may very often have a great impact on the identification, treatment needs, functional status, and progress of those effected (Gillberg \& Billstedt, 2000). Individuals with autism may have a range of behavioral symptoms, including hyperactivity, short attention span, impulsivity, aggressiveness, self-injurious behaviors, and temper tantrums, particularly in young children (American Psychiatric Association, 2005).

Autism Spectrum Disorder (ASD) is a disorder that strikes early in life and lasts throughout the lifespan. Based on the findings in available follow-up studies, only a small percentage of individuals with ASD are able to live and work independently as adults. About one-third of affected individuals may be able to achieve some degree of partial independence. Even the highest functioning adults with ASD typically display problems in social interactions and communication along with noticeably restricted interests and activities (DSM-IV-TR, 2005). A fortunate minority of parents may have the finances for costly private care and training for their children. However, the cost of aides, tutors, and special schooling can be prohibitive for many parents. Even though parents may spend a great deal of money for various therapies for their children with autism, they may still experience high levels of stress. 


\section{Parent Stress}

Most of the parents who have learners with autism bear more stress than parents of typically developing children (Dyson, 1993; Mancil, Boyd, \& Bedersem, 2009; McGrath, 2006; Wolf, Noh, Fisman, \& Speechley, 1989) and children with other development deficits (Boyd, 2002; Bristol \& Schopler, 1984; Morgan \& Sanders, 1997). Accordingly, parents of children with ASD consistently report more symptoms of anxiety and marital dissatisfaction than parents of children with other development delays or disorders (Dunn, Burbine, Bowers, \& Tantleff-Dunn, 2001; Holroyd \& McArthur, 1976; Konstantareas \& Homatidis, 1989). A longitudinal study by Gray (2002) tracked 31 Australian children and their parents for 10 years and discovered that as the age of children with autism increases, the number of support options usually decrease. Minnes and Woodford (2005) also reported that $65 \%$ of parents expressed age-related changes adversely impacted their life. As children with ASD get older, parents have anxiety about their future. In addition, parents of children with ASD also report less involvement in recreational and sporting activities, which may cause social isolation and subsequently higher stress levels (Mancil, Boyd, \& Bedersem, 2009).

Mancil, Boyd, and Bedersem (2009) reviewed 19 studies related to parental stress and concluded that a number of researchers have started to introduce coping strategies for parents to learn to cope with the stress pertaining to raising a child with ASD. However, the review found that there was not any strategy that consistently contributed to reducing or coping with parental stress. Some strategies may temporarily provide positive outcomes but may produce unexpected long- term outcomes and resulted in stress later that may be much worse in severity, for example, using social withdrawal and keeping children separated from siblings (Quill, 1995).

Obviously, the relationship between parents and their children will impact the treatment results for individuals with autism. The most effective treatments for individuals 
with autism will also provide support and training for parents (Lovaas, 1987). Tailoring effective teaching and interaction strategies for successful parent training is important to the success of interventions and the reduction of parents' stress. Parent training definitely enhances parental self-efficacy in interacting with their learners. A parent's sense of effectiveness or self-efficacy has been identified as the strongest predictor of the degree of parent involvement in the intervention (Solish \& Perry, 2008). A parents' feeling of selfefficacy in helping their children with autism will reduce their overall stress levels. One such method of parent training is called Applied Behavior Analysis.

\section{Core Concepts of Applied Behavior Analysis}

Applied Behavior Analysis (ABA) is defined as "a scientifically based technology of behavior change which emphasizes learning as a cause of behavior" (Jenson, Sloane, \& Young, 1988). The concepts and principles of ABA focus on effective instruction and management provided by trained professionals who have such knowledge and are able to apply the behavioral techniques (McCuller, 2002). ABA is built on the substantial empirical foundations of experimental research and applied research. It has provided the evidencebased treatment of choice of individuals with autism (Shook \& Neisworth, 2005). A larger number of studies support the efficacy of specific intervention strategies, most of which are based on Applied Behavior Analysis (Rogers, 1998; Atwell, Wilder, \& Wine, 2006; Grindle et al., 2012). In short, ABA has been described as one of the most common and only evidence-based method used to treat learners with autism to produce positive outcomes for their undesirable behaviors.

To address the needs of parents and children, research has demonstrated that the use of superior teaching strategies can overcome many learning difficulties caused by disadvantaged environments (Binder \& Watkins, 1990; Watkins, 1988). Superior teaching strategies can promote the success of learners having a variety of learning challenges. There 
are many treatments for helping learners with autism; however, Applied Behavior Analysis is currently considered by various scientific organizations, including the National Institutes of Mental Health, National Academies Press, Association for Science in Autism Treatment, Organization for Autism Research, and Autism Speaks, as the most powerful educational approach and producing the most effective positive outcomes in helping reduce the main symptoms for learners with ASD. There is also a large body of research that has been published to support the efficacy of implementing ABA with learners with autism (Iwata et al., 1994; Lovass, 1987; Koegel, Koegel, Harrower, \& Carter, 1999; Whitaker, 2002). In other words, the concepts and principles of ABA have been used to successfully improve the learning repertoires for large numbers of learners with autism based on the evidence shown from different studies.

Forehand et al. (1979) and Koegel, Glahn and Nieminen (1978) found that teaching parents general behavioral principles and giving them a basis for developing new interventions as needed was a more effective approach than approaches that teach parents specific interventions for a particular problem behavior (as cited in Koegel, 2003). Parent training varies, but most programs have some basic similarities. The three core concepts of reinforcement, prompting, and fading have been frequently applied by behavior analysts and also are easily taught to parents. There is a rich history in research about the usage of these three techniques in interventions for learners with autism.

Lerman, Perkins-Parks, Roane, and Swiezy (2000) studied three families to assess the impact of management strategies for parents. The management program included withholding attention from undesirable behaviors, applying verbal prompts when the learner failed to respond, and differential reinforcement for no occurrences of undesirable behaviors. Parents learned to use instructional prompts following noncompliance to provide reinforcement 
following compliance with their instructions. The results showed that all parents successfully learned the skills through written and verbal training along with a coach's feedback.

Stahmer and Gist (2001) studied 22 families during 12 weeks of training. Parents learned the timing for giving reinforcement and differentiated the proper condition for delivering reinforcement. The training focused on the usage of reinforcers by parents who successfully motivated improvements in their learners' language abilities. Another study conducted by Ingersoll and Dvortcsak (2006) recruited nine families with learners ages three and four and diagnosed with ASD. The trainers combined direct and indirect techniques in the study. Direct interventions included naturalistic and behavioral interventions, which consisted of incidental teaching, milieu teaching and pivotal response training. The trainers also applied prompting and reinforcement to establish social-communication skills with learners. Parents learned to use prompting and reinforcement to increase acceptable behavior, decrease inappropriate behavior, improve family interactions, and support a positive family atmosphere.

McLaughin, Peterson, and Weber (2008) conducted research regarding a MLT technique combining visual prompts paired with fading to teach a 13-year-old when learning the concept of "Where". The study results showed that the participant maintained this skill for identifying correctly the nine locations at school even when visual prompts were systematically reduced during the fading procedure.

These studies have demonstrated that reinforcement, prompting, and fading concepts have been widely introduced in the field and especially in parent training programs. These techniques are not only frequently implemented by behavior analysts and other professionals, but have also been successfully taught to and used by parents. 


\section{Parent Participation in the Training of Their Children}

Training parents to be co-therapists with their own children is one approach to family involvement in the Early Intervention movement that started in the late 1970s and early 1980s. After "Part H" of the Education of the Handicapped Act Amendment of 1986 (Public Law 99-457) was enacted, parent training was emphasized more than it had previously been. Therefore, this approach was increasingly applied and studied (Welterlin, 2009).

In fact, parents acting as therapists or tutors for their own children is not a new concept. Parents have always played a role in controlling, guiding, and teaching their offspring. Lovaas and colleagues in a 2-year study provided intensive treatment for 20 learners with autism who were one (1) to four (4) years old and first emphasized the importance of training parents as co-therapists for learners with ASD. The study discovered that children whose parents were trained to carry on the intervention continued to make gains, whereas children who returned to an instructional setting without parent involvement lost their previously acquired skills. The study demonstrated the effectiveness of behavior analysis procedures and showed that parent-training programs are effective in the treatment of children with special needs (Lovaas, Koegel, Simmons, \& Long, 1973). Since then, parents of learners with ASD have been successfully taught a variety of intervention techniques for ameliorating the parent-child relationship, improving problem-solving abilities, and preventing conflict in the home. Teaching parents to provide the intervention has also been shown to increase generalization and maintenance of skills over time (Koegel, Schreibman, Britten, Burk, \& O’Neill, 1982). It has also been shown to decrease inappropriate behaviors of their learners (Marcu, Lansing, Andrews, \& Scholper, 1978).

Parent training also improves the quality of life for the family by reducing parental stress (Kergel et al., 1996). 
Another investigation by Lovass (1987) studied two groups of learners with autism who were between 30 and 46 months for at least two years. One group experienced 40 hours of one-on-one based service each week and another group experienced 10 hours or less of one-on-one intervention per week. The treatment goals for each year were different and included reduction of self-stimulatory behaviors and aggression during the first year, developing verbal expression, abstract language, and interactive play skills during the second year, and teaching emotions and pre-academic skills during the third year. Clear changes favoring the 40 hours per week group were shown at the beginning of the second year. These study results support the importance of early intensive behavioral intervention for learners with autism.

A study by Sheinkopf and Siegel (1998) conducted a pretest-posttest home-based parent training program with the assistance of community-based clinicians that continued for less than two years (mean $=16$ months). For the experimental treatment group, parents received training and implemented behavioral interventions with their learners. A control group only received school-based intervention and brief one-on-one service. The results showed that on IQ tests the learners in the home-based intervention scored 25 points higher than those in the school-based treatment following treatment. The importance of these findings is that intensive home-based behavioral treatment can be successfully accomplished without the extra support from an academic center. The results also showed that home-based treatment programs of fewer than 30 hours per week could improve IQ scores more than school-based programs.

Another research investigation by Jones (2009) used a method based on a study by Kaiser et al. (1995). In this study the primary researcher combined Discrete Trial Training (DTT) and Incidental Teaching (IT) and only trained one parent directly. Then this parent provided training to the other parent using the same materials. Three families were recruited 
including both parents and their children. The results of this study showed that parents could learn to successfully implement behavioral techniques and transfer what they have learned to other parents.

Welternlin (2009) recruited 10 learners with autism and their families for a Home TEACCHing Program that lasted 12 weeks for 1.5 hours per week. The children participants' adaptive behavior, problem behavior, and learning skills were examined pre and post intervention. Parents in the treatment group received training based on the TEACCH model. Parents met trainers at home to apply the techniques with their two or three year old learners across a variety of curriculum settings. The results indicated parents were able to learn from the Home TEACCHing Program and increase their abilities to appropriately interact with their learners and to implement effective prompts to increase learners' desirable behavior and reduce maladaptive behavior. Parent stress and anxiety also decreased as compared to the waitlist group.

Raising a child with autism can be challenging, and often exhausting, frustrating, and heartbreaking for parents (Heward, 2006). Current information pertaining to families with learners with autism indicates a need for parent training. There is wide spread agreement that parents are an important factor and play an essential role in furthering the development of their children. If the rate of children diagnosed with autism increases every year, it is critical, then to ask whether parents' capacity to help their learners with autism is likewise increasing. According to our current understanding of autism, we know it will likely be a lifelong disorder that requires a high burden of care. Therefore, having early identification and intervention in place to maximize positive outcomes is very important (Bryson, 1996). Obviously parent behavior is an important environmental variable contributing to children's development. Parents' consistent application of proper strategies will allow the learner to significantly improve in the performance of various life skills, including self-care, academics, 
and social skill repertoires. Many children with autism require different learning conditions than most other learners. They require prudently planned, precisely delivered, successively evaluated, and systematically analyzed instruction (Heward, 2006). Therefore, it is important to introduce parent training to have well prepared and skillful parents helping their learners consistently, especially in early intervention programs. According to the National Research Council (2001), parents generally are the most important component influencing their children's development and learning in their daily environments. Without the involvement of parents, the gains at home for individuals with autism are limited. Effective parent training promises to assure improvements in their learners' communication behavior, increase parental knowledge, enhance parent-child interaction, improve their communications with their learners, and reduce the parental stress and depression linked with having a learner with autism (Aldred et al., 2004; Hastings et al., 2005; Koegel et al., 1996; McConachie \& Diggle, 2007).

Research about academic success of children indicates that parent involvement is a major factor in their children's learning outcomes (Desforges \& Abouchaar, 2003). Roberts and Kaiser (2011) found that when parents actively used language intervention techniques, the receptive and expressive language skills of their children were positively affected, whether the children had intellectual challenges or not. In addition, parent tutoring has been successfully used to increase children's oral reading fluency. Therefore, engaging parents in their children's learning processes provides a valuable addition to improving the children's academic success (Kupzyk, McCurdy, Hofstandter, \& Berger, 2011). Parent involvement improves the generalization of their children's language as well (Adamson, Bakeman, Romski, \& Sevcik, 2010). It has been well established that parents can play an instrumental role in encouraging their children's writing development (Aram \& Levin, 2001; Neumann \& Neumann, 2010). Based on Vygotsky's concept of "parental scaffolding" in children's 
learning, parents' involvement has been shown to benefit children's learning progress (Pratt, Kerig, Cowan, \& Cowan, 1988; Pratt, Green, MacVicar, \& Bountrogianni, 1992; Ward \& Zembal-Saul, 2007).

Parent training should be one of the priorities in present day education. Many parents can develop good child raising skills on their own, but many more cannot. However, with parent training, better parenting skills can become more widespread more quickly for many parents, especially those who have learners with special needs (Diouf, Sheckley, \& Kehrhahn, 2000). Typically, parents want to be a part of their children's overall learning achievement; however, they are frequently unaware of the most appropriate or effective ways to get involved (Luong, 2008). Therefore, providing training and planned learning experiences for parents who have learners with autism can be an effective way for parents to develop the skills necessary to support their children's learning. Once parent training has been accomplished, the cost of special needs children to the local, state, and federal governments can decrease. Additionally, as the parents themselves implement the training, their levels of stress and frustration may also be reduced.

The use of parent training to help them deal effectively with their children's behavioral problems has been evaluated in numerous studies. These studies developed parent training approaches, allowing them to become "co-therapists" in their children's treatment, reinforcing what the child experienced in "out-of-home" therapy, and/or providing all the therapy a child might receive. The needs of many children with autism are so overwhelming and pervasive that parent training evolved as a response to the need. Once the parents are given the tools to teach their own children, the results showed that the children were better equipped with necessary skills and there was a decrease in inappropriate behavior (Koegel, Brookman, \& Koegel, 2003). In addition to helping the parents interact with their children in a more positive and beneficial way, the training helped parents learn how to apply teaching 
and training techniques to facilitate their children's skill acquisition. Another positive outcome of parent training programs is the aspect of being "cost effective" and the efficiency of having this trained parent/therapist with the child for a large percentage of the child's waking time.

According to research, the most effective training programs for children with autism are those which have been tailored for the particular child, addressed communicative intent of child behavior, and prompted social reciprocity between children and individuals with whom they have regular contact (Elder, 2002; Koegel \& Koegel, 1987; Koegel, O’Dell, \& Koegel, 1987; MudFord, Martin, Eikeseth, \& Bibby, 2001; Mundy, Sigman, \& Kasari, 1994).

Obviously, parent training has been considered a critical component of successful intervention programs for learners with autism. And usually parent involvement produces more favorable outcomes (National Research Council, 2001).

In short, parent education efforts have shown that parents can be taught various teaching techniques and implement effective interventions with their own learners. However, a parent, even with the best of intention, may find it overwhelming to tackle the problem of providing appropriate assistance to a special needs child without help and support. Reading a book, finding information on the internet, or consulting with other parents who have similar concerns can be beneficial; however, having assistance or guidance from trained professionals can be very invaluable in improving the teaching skills of the parent learner.

\section{Competent Learner Model}

Since professional training needs to increase, the Competent Learner Model (CLM) Course of Study $(\mathrm{CoS})$ has been introduced for caregivers, parents, and teachers based on the CLM already in existence for the last 30 years. The Competent Learner Model Course of Study provides additional training that bridges the gap between theory and practice in teaching individuals with autism, because it represents research and performance-based 
training. Behavioral treatment has been described as one of the most common, and only evidence-based method used to treat learners with autism. Therefore, Vicci Tucci and colleagues have established the Competent Learner Model (CLM) Course, which is based on Applied Behavior Analysis, and merges Precise Teaching (PT) and Direction Instruction (DI), for helping people to practice the strategies and concepts more effectively in teaching individuals with autism. Both Precision Teaching and Direct Instruction have been reported to be among the most powerful instructional techniques when compared to other traditional teaching practices in helping students to achieve their learning. Also, researchers have suggested that Precision Teaching and Direct Instruction might be used to reduce the skills crises in American regular and special classrooms, if widely applied (Binder \& Watkins, 1990). Applied Behavior Analysis is defined as "a scientifically based technology of behavior change which emphasizes learning as a cause of behavior" (Jenson, Sloane, \& Young, 1998). The Competent Learner Model has an established track record of more than 30 years for helping learners to build learning repertoires. This teaching method is based on B.F Skinner's analysis of learning behavior and has been organized by Vicci Tucci and colleagues. Prior research reports that CLM has effectively served numerous children with a variety of learning challenges, including pervasive developmental disorders, challenging behavior, and Autism (Hursh, Laitinen, \& Tucci 2004). As we know, a superior teaching method can increase the probability of achieving desired learning outcomes. The Competent Learner Model is one of the most powerful teaching concepts available for helping learners.

The Competent Learner Model (CLM) has been used to serve numerous children with a variety of learning challenges, including pervasive developmental disorder, challenging behavior, and autism (Hursh, Laitinen, \& Tucci, 2004). Through the use of coaches, the Competent Learner Model Course of Study provides additional training that bridges the gap between theory and practice in teaching individuals with autism, by applying research-proven 
methodology to performance-based training. Trainees watch demonstration videos and work with coaches who model and assist the trainees in implementing the teaching concepts they see demonstrated.

The CLM Course of Study provides a coaching process that helps trainees arrange and rearrange instructional conditions so as to establish, strengthen, and maintain their learners' competent learning repertories. In the beginning, the purpose of designing the CLM Course of Study was to train educators and parents for teaching an individual to be a competent learner who observes, listens, talks, reads, writes, participates, and problem solves effectively (Hursh, Laitinen, \& Tucci, 2004). The coaching system in the Course of Study is a very significant component of tracking the progress and results of each learner.

The CLM Course of Study contains one introductory and twelve instructional units. Trainees participate in self-paced training while mastering each unit in succession. Trainees learn a clear curriculum and, upon completion, will have the skills necessary to establish in their students the seven repertoires: observer, listener, talker, reader, writer, problem solver, and participator (Tucci Learning Solutions, Inc., 2006). Since coaching is one of the essential elements in the Competent Learner Model, quality coaching is vital. Therefore, a coach model may be an effective teaching strategy for parent training that supports the ongoing needs of each family and increases the likelihood that parents can accomplish intervention plans with a higher degree of procedural accuracy.

\section{Coaching Benefits}

Due to different needs of each learner, each family, and the learning mechanism of adults, a coaching model may be an effective approach to supporting parent training. Coaching is a means that may bridge the gap between what parents know and what they apply of what they know (Fixsen, Naoom, Blasé, Friedman, \& Wallance, 2005). 
Effective coaches incorporate the prior experiences of their adult learners into their coaching methods. Research by Guiney (2001) and Britton and Anderson (2010) supported the use of the coaching model in adult learning. Adult learning theory states that adults must be allowed to move through the learning process at their own pace. They also need to be allowed time for repetition and guided practice in their new skills. In fact, adults often need to unlearn old habits and replace them with new alternative behavior. Therefore, reflecting upon their existing habits is important when encouraging the development of new skills. Coaching can aid in this process (Shidler, 2009) because internal processes and external interactions between the instructor and the learner can shape the learning process (Greer, 2002; Moran \& Malott, 2004). Therefore, application of a model of coaching that includes discussions of the experiences while providing support in the environment can be very helpful (Akyeampong, Pryor, \& Gharty, 2006).

Coaching has been widely applied as an effective system of providing personal development. The coaching role involves mentoring adult performance and providing personal development to assist individuals in mastering the strategies being implemented. Moreover, an effective coach observes and provides non-evaluative feedback on individual implementation of strategies. Coaches are not present to "fix" the individuals, but rather to collaborate with them to develop what will work in the environment for their learners (Blamey, Meyer, \& Walople, 2008). Most coaches provide guidance, advice, modeling, and support for adult learners in establishing more opportunities for their learners to achieve and engage (Mohler, Yun, Carter, \& Kasak, 2009). The coach is able to assist adults in learning with respect to their unique personal and professional development. According to social learning theory, learning takes place best through observation and modeling in social settings such as family, workplace, and school when the actions and responses that are modeled for observation have meaningful context. 
Research also has found that in a learning setting, immediate feedback has a greater influence on individuals' behavior than traditional feedback that was provided hours, days, or even weeks after an adult was observed (Coulter \& Grossen, 1997). In addition, researchers stressed that delayed feedback might create the possibility for individuals to learn and practice their errors and shape the wrong behaviors (Heward, 1994; Malott \& Suarez, 2004). Reinke et al. (2007) conducted parent training with a coaching model helping parents in the usage of clear commands, specific praise, and descriptive play statements. A coach provided a handout with visual performance feedback each session so that parents could monitor the result of their own learning.

Keller (2007) found that when a school district introduced coaching for teachers, it worked better than workshops and courses for guiding participants to achieve their goals because it helped teachers to problem-solve in real time. Furthermore, a good coach is able to decide which form of feedback is most beneficial for the trainee and select the best timing for providing that feedback. As a result, the coaching model showed the most cost-effective way to build competency for these teachers.

Research results have indicated the importance of social interaction, observation of social roles, and the significant role of mentors in the learning process (Bandura, 1970). When the learners are parents of children with special needs, the best way for them to learn useful strategies is through practice in order to achieve mastery as they are observing and applying the new skills demonstrated by coaches. Therefore, in parent training, the vivo coach's role in improving parent learning may be accomplished through model-lead-test strategies.

Model-Lead-Test. Model-Lead-Test (MLT) strategy instruction (Marchand-Martella, Slocum, \& Martella, 2004) is a three-stage coaching process used for teaching students to independently use learning strategies: (1) instructor models correct use of a strategy, (2) 
instructor leads learners to practice correct use, and (3) instructor tests learners' independent use of the strategy. Once learners attain a score of $80 \%$ correct performance on two consecutive tests, instruction in the strategy stops.

A Model-Lead-Test teaching paradigm has been used to shape comprehension responses to a level of independence, without instructor assistance (Idol, 1987). More recent studies have supported the effects of MLT in teaching a wide range of repertoires for learners with a variety of disabilities (Derby \& Johnson, 2011; Glover, McLaughin, Derby, \& Bower, 2010; Green, McLaughlin, Derby, \& Lee, 2010; Kaufman, McLaughlin, Derby, \& Waco, 2011; Ruwe, McLaughlin, Derby, \& Johnson, 2011). The current studies are more focused on using MLT with younger learners instead of parents. However, the techniques used can work well with parents when modified for adult learners.

A study by Shouse, Weber, and McLaughlin (2012) evaluated the effects of model, lead, and test procedures to teach a preschooler with a disability to identify colors via an AB-A multiple baseline across colors design. A 2-year 5-month old child with developmental delays was the participant. During the baseline 1, the participant was shown two different colored bears at a time. The researcher then gave the participant an instruction to pick a specific color bear. As long as the learner selected either bear in response to every verbal instruction, he was allowed to bounce on a ball at the end of the session. In the MLT treatment phrase, the learner was also presented with two bears of different colors. If the learner pointed to the right color, the researcher praised him. If the learner pointed to the wrong color, the researcher implemented the MLT procedure and the learner was allowed to bounce on the ball at the end of the session. Baseline 2 procedures were the same as for baseline 1 . The outcomes of this study showed the combination of MLT and a reward was an effective tool for teaching color recognition. 
Peterson, Mclaughlin, Weber, and Anderson (2008) conducted a study to assess the effects of the MLT technique with visual prompt paired with a fading procedure to teach the "where" concept to a 13 year old echolalic learner with autism. The study was conducted by using the MLT technique in the participant's self-contained special education classroom and in nine different locations around the school. The participant successfully learned to identify the nine various locations and maintained the skill even after the visual prompts were faded.

According to Billet (1994), the goal of on-the-job training or apprenticeships for skilled vocations is to help trainees develop and internalize work skills and knowledge, and then be able to implement them independently in the work place. Billet further stated that such situated learning relies on interplay between observation, scaffolding, and the increased independence of learners. As an instruction method, apprenticeships may involve four phases: modeling, coaching, scaffolding, and fading that are equivalent to the model, lead, and test strategy.

Modeling is an imitation process and a demonstration of the temporal process of thinking (Dennen, 2004). Applied behavior analysts demonstrate or model the desired behavior as a response prompt (Cooper, Heron, \& Heward, 2007). According to Billet (1994), modeling requires an expert to execute a task so that the learner can observe and build a conceptual model of the process involved in successfully accomplishing the task. However, the expert must also verbalize his or her thinking. Modeling prompts the learner to visualize the task implementation. Without having the time to sensitize instructors to reveal their cognitive processes, modeling enables learners to absorb the tasks, so that tasks are done according to specifications. An experiment based on Knowles' theory of andragogy and Bandura's theory of modeling has shown better results for adult learning using methods based on these theories than with other previously used instruction methods (Frazier-Verner, 2010). Cohen (1995), in his research on providing proactive models in learning, concluded 
that his method yielded positive results. Neef, Marckel, Ferreri et al. (2004) examined the effects of modeling for two individuals with typical development and with attention deficit hyperactivity disorder (ADHD). The outcome showed that responding based on modeling was more sensitive to subsequent changes in the reinforcement schedule than responding established with instruction. Research carried out by Werts, Caldwell, and Wolery (1996) also illustrated that peer modeling resulted in learners performing response chains accurately and quickly.

Leading means instructors lead learning, decide what topics should be learned, and what concepts will be taught based on learners' needs. The procedure may result in development of the target skills because it requires experts to observe and monitor learners as they carry out tasks. In addition to feedback, experts may provide hints, clues, and the tricks of the trade. Repeated practices may also be required and may consume additional time. However, in the long run, the learner will be able to apply the concept learned more effectively and independently and with the desired outcome. Leading is similar to scaffolding, which is another teaching strategy and refers to support given from a greater distance (Billet, 1994). Jerome Bruner, a cognitive psychologist, originally introduced the concept of scaffolding in the late 1950s. Initially this term was used to describe young children's language acquisition. The idea of scaffolding is consistent with Soviet Psychologist Lev Vygotsky's work. Interactional support and the process by which adults contrive opportunities for a child to take on new learning have come to be termed "scaffolding". Leading and scaffolding both refer to using support from the teacher when developing a relationship for the leaner's appropriate response to the material or task, after which support is gradually removed to leave behind a fully established response. In other words, leading and scaffolding are the same as a temporary framework for response construction in process (Cazden, 1983). The instructor who provides scaffolding or leading does not change the 
nature or level of difficulty of the task; rather, s/he supports the learner to successfully and independently complete the task. During the leading procedure, instructors have to accurately understand learners' current skill levels. An instructor has to be aware of what content the learner needs to be led to perform, identify the time the learner needs to be led, decide how to implement it, and recognize when the learner is ready to be independent (Lajoie, 2005). Dougherty and Ten (1997) claimed that several studies evaluating scaffolding in the workplace have shown that the investment of time spent in training was regained even when the successful trainees left shortly after mastery.

Model-Lead-Test has been implemented in various learning settings but currently there are no investigations published that explore MLT for use in parent training. Most coaches provide models and feedback during parent training but not the entire MLT procedure. According to Horton (1984), most parent training at that time was accomplished using self-help manuals, lectures, modeling, role-playing, rehearsal, feedback, or a combination of these methods.

Raj and Salagame (2010) conducted a study comparing the existing coaching model at the participating agency to a "sensitized model" (i.e., an enhanced version of the existing model that contained specific sensitivities infused into the coaching practices of modeling, rehearsal, and feedback) and measured this sensitization by Johnston and Mash's (1989) version of Gibaud-Wallston and Wandersman's (1978) Parenting Sense of Competence Scale (PSOC). The coaches for the sensitizing parent-training group were trained to fade their support systematically, so parent participants could experience repeatedly succeeding on their tasks, which would in turn promote their perception of competence, while at the same time lessening dependence on the coach. The purpose of this study was to evaluate and compare the self-efficacy of the two parent training groups. Parents in each group were measured on the behavior of domain-general and task-specific self-efficacy, both before and after training. 
Overall, the results of this study supported the use of sensitizing parent-coaching models to enhance mother-participants' task-specific self-efficacy.

Welterlin (2009) used didactic training, modeling, and hands-on-training, which is also similar to the MLT procedure, during the parent education procedure in his study. Two parent participants showed no treatment effects until after beginning the last treatment probe, which was the hands-on phase. One possible interpretation of this finding could be that these two participants learned more effectively when taught with hands-on training as opposed to modeling only. Or it could be that the experience of both modeling and hands on training, much like MLT, is a more powerful parent training procedure. This remains an empirical question that this investigation is designed in part to answer.

\section{Conclusion}

Based on the Centers of Disease Control and Prevention's 2014 estimation, 1 out of 68 children has Autism Spectrum Disorder. The rate has risen 25\% since 2008. Due to the rapidly increasing rate of individuals being diagnosed with Autism Spectrum Disorders in recent decades, introducing adequate teaching strategies and concepts will be very important for assisting parents and caregivers in providing more consistent intervention at home. These strategies will become powerful resources in generalizing skills across different settings, people, and items to promote the ability of children with Autism Spectrum Disorder to learn and communicate more appropriately. The rationale for using Applied Behavior Analysis is that the preponderance of previous studies using evidence-based therapies shows that ABA has proven to be one of the most effective therapies to date. In the past, Model-Lead-Test strategies have been successfully used with learners with a variety of disabilities and are more recently being used with younger learners. The researcher chose to use similar yet modified MLT techniques with parents. The single subject research design is also employed in this study as the methodology of choice because it has proven to be a superior quantitative 
approach to examining the functional relationships between baseline and experimental conditions over time within individual learners (Kazdin, 2011). As this study represents the outcomes of a small number of subjects with autism, a condition which represents a minority within the population, the single case versus the larger number studies, proves to be the best methodology.

\section{Statement of Purpose}

The purpose of this study is to evaluate the effectiveness of the core content of Applied Behavior Analysis for parents of learners with Autism Spectrum Disorder through Model-Lead-Test training via a multiple-baseline across behavior design. 


\section{Research questions}

Research Question 1. Is Parent training using a Model-Lead-Test procedure effective for increasing parents' appropriate use of reinforcement, prompting, and fading techniques?

Research Question 2. Do the learner's talker, participator, and problem solver repertoires improve for learners with developmental delays as their parents' appropriate use of reinforcement, prompting, and fading techniques increase? 


\section{Chapter Two}

\section{Methodology}

\section{Participants}

Participants included 3 children from eight to ten years of age with diagnoses of Autism Spectrum Disorder and their parents. They were recruited through a local agency, Tucci Learning Solutions, which provides behavior intervention services to learners with special needs and their families. The following criteria were used to select participants: (a) the family has not previously received systematic in home parent training, (b) either the mother or father has agreed to participate in a general parent education curriculum, (c) the learner has a documented diagnosis of Autism Spectrum Disorder or other learning development delays based on current diagnostic criteria, and (d) the learner has Competent Learner Repertoire Assessment (CLRA) results that place him/her between the pre-1 level and Level 3 within the CLRA. Competent Learner Repertoire Assessment is one component of Competent Learner Model, which was invented by Vicci Tucci (2004). The researcher contacted parents of learners who meet eligibility criteria to get permission for the study. Behavior specialists will be asked to contact parents of children who meet eligibility criteria to get permission for the researcher to contact their families. They were given a brief script that provides an overview of the intervention and research project. Once the behavior specialist has verbal permission for the researcher to contact parents, the researcher will contact interested families to explain the intervention and study in greater detail. If the parents were interested in participation, the researcher set up a time to meet with the family to administer the Competent Learner Repertoire Assessment, identifying each child's learning repertoires to determine their eligibility to participate. During the meeting, the researcher explained the consent form to the parents and acquire their written consent in order to proceed. All child participants were assessed using the Competent Learner Repertoire 
Assessment (CLRA) in order to characterize their skills in different repertoires and gain a pre-intervention score. There were no eligibility criteria for parents except that they have not previously received systematic parent training in the application of Applied Behavior Analysis with their child. Parents were informed whether or not their child meets the eligibility criteria once the CLRA is scored. For those eligible children, the researcher set up a schedule for behavior observations and parent training.

Three to five available parent-child dyads were randomly selected to participate after reviewing and signing a consent letter. The child participants in these families all ranged in age from 4 to 12 years and parents had reported that they all had qualitative impairments in communication, demonstrate lacking motivation for participation with non-preferred activities, and difficulties in self-care skills. For child participants in this study, CLRA levels ranged from Pre-1 to 3.

\section{Setting and Materials}

The training and observations took place in the families' homes. The parents and researcher will choose the quietest and most minimally distracting area in the house for training and observations. Usually it took place in either a family living room or the learner's bedroom. The primary researcher stayed in the room with the parent and child participants during baseline, training, and maintenance sessions. When the session was conducted, parent and child work on the tasks at a table, which only contains materials provided by the primary researcher. All of the distractions will be eliminated as much as possible, such as background noises, TV, and music. All of the reinforcers were placed in a plastic container and available to be applied as needed. Prior to training, parents were supplied with a timer, training script, calendar, and a home teaching kit. The home teaching kit consisted of three to five selfcontained tasks designed to teach learners with learning development delays a variety of communication skills that made up talker, participator, and problem solver repertoires. 
During training, parents received a written description of the training content and implementation protocol. A video recorder was used to record the events during the training and behavior observation sessions. This video recording was later be used to compare against other observations in establishing a high inter-observer agreement, IOA, for the study.

During the experimental session, the researcher set up the video camera on a surface so that it was facing the training table. The researcher positioned herself during observations so that she would not in plain view of parent and child participants when they were interacting but she could still easily observe and record them. When the researcher begins the video, the exact time was recorded and the researcher begins to record the interactions of the parent and child participants as outlined in the studies protocol. Likewise, the stop time was recorded once the researcher determines the data collection was completed.

\section{Independent Variables}

The independent variables included parent training (Oral Lecture/ Discussion [OLD] of ABA core concepts and coaching procedure/Model-Lead-Test [MLT]) and maintenance (follow up-data collection only). Details were described below. The sequence of events were as follows: baseline, parent training I (OLD or MLT), parent training II (MLT or OLD), and post training data collection (Maintenance phase).

The Oral Lecture/Discussion Training for this study consisted of three sessions regarding the processes of reinforcement, prompting, and fading. The training sessions were conducted in a one-on-one format. One process were presented during each training session. Each process was defined below in Table 1. 
Table 1

Definitions for Each Process

\begin{tabular}{|c|c|}
\hline Process & Definition \\
\hline Reinforcement & $\begin{array}{l}\text { Reinforcement is a core concept of ABA and describes a relationship } \\
\text { between two environmental events, a behavior (response) and an } \\
\text { event or consequence that follows the response. The relationship is } \\
\text { termed reinforcement only if the response increases or maintains its } \\
\text { rate as a result of the consequence. Applied behavior analysts apply } \\
\text { the principle of Reinforcement to modify behavior in a systematic } \\
\text { manner. }\end{array}$ \\
\hline Prompting & $\begin{array}{l}\text { Prompting is adding a stimulus that increases the probability that a } \\
\text { discriminative stimulus }\left(\mathrm{S}^{\mathrm{D}}\right) \text { will evoke the desired behavior. }\end{array}$ \\
\hline Fading & $\begin{array}{l}\text { Gradual removal of prompts is referred to as fading. Any prompt } \\
\text { may be systematically faded so the response occurs and is reinforced } \\
\text { when the discriminative stimulus }\left(\mathrm{S}^{\mathrm{D}}\right) \text { alone is presented. }\end{array}$ \\
\hline
\end{tabular}

The primary researcher was a Board Certified Behavior Analyst who had led several parent-training groups in Taiwan and had frequently conducted individual parent training sessions in her current position before beginning this study. The procedures in the oral lecture/discussion training required that parents receive the training in a one on one training format where the researcher presented and discussed the definition, importance, and implementation of each process. The presentation and discussion of each process took about 45 to 60 minutes. The total training was three sessions for each parent participant, one for each process. During the presentation session, the researcher provided information about each topic and engaged the parent participant in discussion surrounding the topic. The parent participant decided the protocol for implementing each skill with the researcher based on his/her learner's condition. The purpose of this study was to investigate the efficacy of the core contents of Applied Behavior Analysis (ABA) with parent participants; therefore, only three basic concepts of ABA will be trained: reinforcement, prompting, and fading. Table 2, below, outlines processes and activities covered in each instruction session. 
Table 2

Process, Content, and Activities for Each Presentation Session

\begin{tabular}{|c|c|c|}
\hline Process & Key Content & Activities \\
\hline Reinforcement & $\begin{array}{l}\text { 1. Definition of reinforcement } \\
\text { 2. Define parameters of reinforcement: } \\
\text { timing, quantity or magnitude, duration, } \\
\text { and variety } \\
\text { 3. The importance of reinforcement } \\
\text { 4. The schedule of reinforcement } \\
\text { 5. Implementation of reinforcement } \\
\text { with learner's program }\end{array}$ & $\begin{array}{l}\text { 1. Oral lecture from } \\
\text { researcher } \\
\text { 2. Discussion of how to } \\
\text { apply reinforcement in the } \\
\text { child participant's program } \\
\text { 3. Decide a protocol to } \\
\text { deliver reinforcement for } \\
\text { the program }\end{array}$ \\
\hline Prompting & $\begin{array}{l}\text { 1. Definition of prompting } \\
\text { 2. Types (hierarchy) of prompting: } \\
\text { verbal, gesture, point, model, written, } \\
\text { and physical } \\
\text { 3. 3-step prompting procedure which is } \\
\text { comprised of changing progressively } \\
\text { through a verbal prompt, model/gesture } \\
\text { prompt, and physical prompt } \\
\text { (Miltenberger, 2004) } \\
\text { 4. Errorless prompting } \\
\text { 5. Implementation of prompting with } \\
\text { learner's program }\end{array}$ & $\begin{array}{l}\text { 1. Oral lecture from } \\
\text { researcher } \\
\text { 2. Discussion of how to } \\
\text { apply prompting in the } \\
\text { child participant's program } \\
\text { 3. Decide on a protocol to } \\
\text { apply prompting in the } \\
\text { program }\end{array}$ \\
\hline Fading & $\begin{array}{l}\text { 1. Definition of fading } \\
\text { 2. The importance of fading } \\
\text { 3. Fading techniques based on the } \\
\text { hierarchy of prompting (review the } \\
\text { types of prompting and most to least } \\
\text { prompting) } \\
\text { 4. Implementation of fading with } \\
\text { learner's program }\end{array}$ & $\begin{array}{l}\text { 1. Oral lecture from } \\
\text { researcher } \\
\text { 2. Discussion of how to } \\
\text { apply fading in the child } \\
\text { participant's program } \\
\text { 3. Decide on a protocol to } \\
\text { apply fading in the } \\
\text { program }\end{array}$ \\
\hline
\end{tabular}

The primary researcher also conducted the Model-Lead-Test (MLT) parent training in this study. Each training session lasted 15 to 30 minutes. During MLT sessions, the researcher demonstrated to the parent participant how to implement the process, then guided the parent to do it with the researcher, and finally let the parent do it on his/her own based on the protocol. The sequence of these activities was as follows: researcher models the technique with the child participant for 5 minutes (model), parent applies the technique with the researcher for 5 to 10 minutes while the researcher provides oral feedback (lead), and parent did it on his/her own for 5 to 10 minutes (test). The criterion for completion was that the 
parent demonstrates correct application of the process three times on his/her own. If the parent did not demonstrate the technique correctly with the child participant after three attempts, then the researcher engaged the parent in role-playing with her to practice the techniques. This prevented shaping any undesirable behavior by the child participant.

The primary researcher followed "Treatment Integrity Checklists" to assure that the details of the training protocols were correctly implemented as seen through the videotaped sessions. The sessions were held in a one on one format; therefore, these forms were used as a guide for the researcher's efforts during each session. The checklists were an elaboration of the outlines of key content and activities provided in Table 4 (Appendix A).

\section{Dependent Variables}

Data were recorded for both parent and child behaviors. The primary dependent variables were the responses per opportunity to properly implement reinforcement, prompting, and fading during the time when the parent participants interacted with their child participants to develop the child's talker, participator, and problem solver repertoires. Throughout the study, videotaped observations were conducted. Each interaction session ranges in length from 10 to 15 minutes. Due to the variation in the durations of the interaction sessions, the responses per opportunity for applying each process was the primary dependent variable used to evaluate the impact of the training procedures on the parent and child behavior.

Data were collected on the following parent behavior: appropriate reinforcement, prompting, and fading. Those responses were selected because they were core techniques of ABA. Increasing appropriate use of those techniques was likely to result in establishing, strengthening, and maintaining the child's talker, participator, and problem solver repertoires.

The responses per opportunity for appropriately applying reinforcement was defined as the number of appropriate applications of reinforcement by parent participants divided by 
the number of opportunities to apply reinforcement when interacting with their child. An appropriate application of reinforcement was defined as parents providing behavior specific praise within three seconds following their child doing what had been defined as an appropriate behavior and following that praise by tangible preferred items within three seconds (see table 3). The primary researcher and the second observer determined if the behavior occurs or not and coded both the opportunity and the correct responses emitted from the participant as seen in the video. Reliability were determined using Inter-observer agreement (IOA) between the two observers.

The responses per opportunity for appropriately applying prompting was defined as the number of appropriate applications of prompting by parent participants divided by the number of opportunities to apply prompting when interacting with their child. An appropriate application of prompting was defined as parents providing an errorless prompt to their child when their child doesn't respond to directives within 1-3 seconds, or responds incorrectly. The purpose of these prompts was to avoid strengthening a chain of errors. If the learner was correct independently in the first response, then she/he immediately received a brief behavior-specific praise instantly followed by a tangible reinforcer from the parent participant. If the learner's first response required a prompt, the tangible reinforcer was withheld and only praise was used. The whole procedure was applied to reinforce independent responses and reduced dependency on prompting. If the child participant did not produce the requested task after the first direction from the parent, the 3-step prompting procedure were implemented to shape the requested behavior.

The responses per opportunity for appropriately applying fading was defined as the number of appropriate applications of fading by parent participants divided by the number of opportunities to apply fading when interacting with their child. An appropriate application of fading was defined as parents implementing partial prompts following previous full prompts 
for their child's target behavior during a 15 minutes interaction session. Operational definitions of parent behaviors are listed below:

Table 3

Operational Definitions of Parent Behavior

\begin{tabular}{|c|c|c|c|}
\hline Parent Behavior & Operational Definitions & Example & Non-example \\
\hline $\begin{array}{l}\text { Properly using } \\
\text { reinforcement }\end{array}$ & $\begin{array}{l}\text { Deliver the reinforcers } \\
\text { within } 3 \text { seconds with } \\
\text { behavior specific praise } \\
\text { identifying desirable } \\
\text { behaviors }\end{array}$ & $\begin{array}{l}\text { Say, "I like your } \\
\text { using words." Then } \\
\text { provide reinforcers } \\
\text { in 1:1 ratio. }\end{array}$ & $\begin{array}{l}\text { Say, "Good job", then } \\
\text { provide reinforcer } \\
\text { independent of the } \\
\text { behavior or provide } \\
\text { reinforcer when parent } \\
\text { prompts the behavior. }\end{array}$ \\
\hline $\begin{array}{l}\text { Properly using } \\
\text { prompting }\end{array}$ & $\begin{array}{l}\text { Use prompt if the } \\
\text { learner cannot respond } \\
\text { independently within 1- } \\
3 \text { seconds and model } \\
\text { the correct answer. }\end{array}$ & $\begin{array}{l}\text { Parent asks, "What } \\
\text { color is the truck?" } \\
\text { Then Says, "yellow" } \\
\text { if the learner doesn't } \\
\text { respond within 1-3 } \\
\text { seconds. }\end{array}$ & $\begin{array}{l}\text { 1. Parent continues } \\
\text { asking, "What color is } \\
\text { the truck? What color is } \\
\text { the truck? ..." }\end{array}$ \\
\hline $\begin{array}{l}\text { Properly using } \\
\text { fading }\end{array}$ & $\begin{array}{l}\text { Fading the full verbal/ } \\
\text { physical/model prompt } \\
\text { by gradually reducing } \\
\text { the model then the } \\
\text { physical guidance to } \\
\text { partial verbal prompt }\end{array}$ & $\begin{array}{l}\text { Using partial verbal } \\
\text { prompt, say, "ye..." } \\
\text { if the learner is } \\
\text { correctly answering, } \\
\text { "Yellow", for } 3 \\
\text { consecutive times }\end{array}$ & $\begin{array}{l}\text { Parent keeps telling the } \\
\text { learner the whole } \\
\text { answer, "It is yellow." } \\
\text { Or Parent keeps } \\
\text { physically assisting the } \\
\text { learner. }\end{array}$ \\
\hline
\end{tabular}

As a secondary measure, the children's behavior were also measured and includes the number of independent responses per opportunity to engage in behavior defined as parts of their talker, participator, and problem solver repertoires. The researcher tallied the number of first responses per opportunity that occurred that meet the criterion for the repertoires.

Operational definitions of child behavior are shown in Table 4: 
Table 4

Operational Definitions of Child Behavior

\begin{tabular}{|c|c|c|c|}
\hline Child Behavior & Operational Definitions & Example & Non-example \\
\hline $\begin{array}{l}\text { CLM Talker } \\
\text { repertoire }\end{array}$ & $\begin{array}{l}\text { An attempt to repeat } \\
\text { parents' sentences or answer } \\
\text { parents' questions within 1- } \\
3 \text { seconds. Based on the } \\
\text { content of CLM curriculum } \\
\text { and each learner's current } \\
\text { Talker repertoire. }\end{array}$ & $\begin{array}{l}\text { Child responds, "I like } \\
\text { pizza and milk." in a } \\
\text { complete sentence when } \\
\text { asked to state two foods } \\
\text { she/he likes with } \\
\text { moderate volume within } \\
1-3 \text { seconds. }\end{array}$ & $\begin{array}{l}\text { Child doesn't respond, or } \\
\text { answers, “...pizza and } \\
\text { milk." with low volume } \\
\text { after } 3 \text { seconds. }\end{array}$ \\
\hline $\begin{array}{l}\text { CLM Participator } \\
\text { repertoire: } \\
\text { Respond to directives } \\
\text { or questions within } \\
\text { two prompts after an } \\
\text { error }\end{array}$ & $\begin{array}{l}\text { Learner responds to a } \\
\text { directive within two } \\
\text { prompts after mistake }\end{array}$ & $\begin{array}{l}\text { Child is told to state two } \\
\text { things about items, e.g., } \\
\text { food you like and its } \\
\text { color. The child states two } \\
\text { things after the error no } \\
\text { more than two prompts } \\
\text { after the mistake. }\end{array}$ & $\begin{array}{l}\text { Child is told to state two } \\
\text { things about items, e.g., } \\
\text { food you like and it's } \\
\text { color. Responding } \\
\text { correctly or incorrectly } \\
\text { after two prompts, or } \\
\text { never responding. }\end{array}$ \\
\hline $\begin{array}{l}\text { CLM Problem Solver } \\
\text { repertoire }\end{array}$ & $\begin{array}{l}\text { Learner solves problem by } \\
\text { manipulating a variety of } \\
\text { different sized and shaped } \\
\text { parts to fit them in place or } \\
\text { remove them from a place }\end{array}$ & $\begin{array}{l}\text { Child brushes teeth by } \\
\text { following through } \\
\text { procedures of picking up } \\
\text { the tooth brush, opening } \\
\text { toothpaste, turning on } \\
\text { faucet, rinsing tooth } \\
\text { brush, turning off faucet, } \\
\text { starting to brush teeth... } \\
\text { Child does each step } \\
\text { within } 3 \text { seconds. }\end{array}$ & $\begin{array}{l}\text { Child doesn't manipulate } \\
\text { parts to solve his problem } \\
\text { in self-care task. }\end{array}$ \\
\hline
\end{tabular}

\section{Experimental Design}

A multiple baseline across behavior design for each parent-child dyad were used to assess the effectiveness of the parent training procedures. Parents identified the period of the day that they were available to interact with their children at home. This part of the day was targeted for observations. The response of per opportunity of ABA techniques used by each parent per session was counted and graphed before, during, and after training. If learners were receiving $\mathrm{ABA}$-based teaching from their behavior service team each week, which focuses on increasing their communication (talker repertoire), socialization skills (participator repertoires) and self-care skills (problem solver repertoires) as well as targets a variety of adaptive behavior, the behavior team maintained the original teaching strategies and did not make any changes associated with the parent training procedures. The behavior team tried not to introduce any new program during the study. However, a log was 
maintained to describe the program in effect for the child during this study so as to notice if there were any changes that might be associated with changes in child or parent behavior measured that might provide alternative explanations for the changes. The ongoing teaching used behavior procedures based on the principles of the Competent Learner Model. The impact of the parent training was evaluated by visual inspection of line graphs of the data for all child and parent behavior previously described.

There were four experimental conditions in this study: Baseline, Oral Lecture/Discussion, Model-Lead-Test, and Maintenance. Prior to beginning the study, the researcher completed a Competent Learner Repertoire Assessment (CLRA) for each child participant to identify the strengths and the weakness of the learner's repertoires. After discussion and agreement, the primary researcher and parent participants selected target goals related to the talker, participator, and problem solver repertoires; therefore, each parent-child dyad might be targeted different behavior. Parent participants learned during the training how to apply reinforcement, prompting and, fading to establish or strengthen their learner's repertoires. Line graphs of data for each child and parent behavior were assessed for changes in mean, level, trend, and variability across Baseline, OLD, MLT, and Maintenance phases to determine the effects of the parent training procedures.

During baseline, each dyad was observed two or three times per week for 10 to 15 minutes for target behavior. During the observations, parent participants were given access to the materials selected for use with their child participant. Parents interacted and directed the child to complete a task based on the plan from the research protocol but did not receive any specific instructions for using the materials. Observations were videotaped and later coded by the primary researcher and a second observer who was blind to which phase is in effect. The second observer randomly selected videos and be given data collection sheets which were designed to apply for Baseline, Oral Lecture Discussion, MLT and Maintenance did not 
indicate any particular training phase (See Appendix M). Therefore, different data collection sheets did not bias the second observer's data. Baseline continues until a dyad demonstrated stable data that reflected the use of reinforcement, which included steady trend and low variability as seen through visual inspection of graphed data points. Steady trend was defined as the general tendency in an upward or downward direction. Low variability was defined as low variance or slight fluctuation between data points as depicted on the graph. The data points represent the parents' use of reinforcement after at least three sessions had lapsed. At that point, the dyad begins the Oral Lecture/Discussion training for learning the first basic concept of Applied Behavior Analysis, Reinforcement. When the data were stable, the Oral Lecture/Discussion training stops. Next, the Model-Lead-Test phase starts. After the data were stable, the Maintenance phase began. Observations were videotaped and later assessed by a second observer for inter-rater reliability. Accuracy of the parent's behavior and the child's correct response per opportunity were both measured.

All parents receive training when the baseline was stable. Training is staggered across different behavior, which included implementing reinforcement, prompting, and fading techniques for the child-participant. Parent training consisted of one-on-one sessions, lasting 15- minutes to 1-hour. Each session the researcher provided a handout to train participants to use behavior techniques with their learners. The training handout was a compilation of concepts and teaching strategies and provided definitions, implementations, and a variety of examples of conditions regarding the target behaviors. Those handouts were available throughout all training phases (See Appendix D, E, \& F).

Training consisted of the Training handout, which incorporates instructions for reinforcement, prompting, and fading techniques, a discussion about each technique and how it could facilitate the development of the target behavior, and a protocol for delivering the technique. The parent attended training sessions for each training topic until mastery was 
achieved. Mastery is attained when criterion performance on understanding the reinforcement, prompt, and fading procedures were all at least $80 \%$ accuracy.

In Oral Lecture/Discussion phase, the parent had to demonstrate correctly answering more than $80 \%$ of the researcher's questions. The primary researcher used accuracy of oral lecture implementation for parent participant checklists to review the learning outcomes for parents (See Appendix G, H, \& I). Most of the questions in the Oral Lecture learning handouts and accuracy checklists were based on the concepts defined in Principles of Everyday Behavior Analysis (Miller, 2006). Underlining was applied to highlight the key words. Later definitions of those terms were asked in the quiz questions. The technique had showed positive outcomes for learning new behavioral concepts with university students (Miller \& Weaver, 1975). If the participant answered the researcher's question independently, then the researcher marked, "yes, independently"; if the participant answered with prompts from the researcher, then "yes with prompts" was marked, and would still be considered correct. If the participant did not answer correctly after two prompts, then "No" would be recorded on the sheet. When the parent participant failed to respond independently either more than 3 consecutive questions or all of the answers were less than $80 \%$ accuracy, then the researcher reviewed the contents for which the parent has made errors with the parent participant.

Once parent had orally demonstrated mastery, then the oral lecture/discussion training was ended but data collection phase was continued until a dyad has displayed a stable trend in the response per opportunity data for the parent's using reinforcement and at least three sessions had lapsed.

In lesson 1, the concept, definition, and implementation of reinforcement was introduced as described in the appendix (See Appendix D). A reinforcer survey form was provided (Tucci, 2006) for the parent to fill out so as to identify potential sources of 
reinforcement for their child. The explanation of reinforcement included when to withhold the reinforcer and the timing of delivering it. The parent received one on one training sessions from the researcher that lasted between 45 minutes and an hour, depending on the amount of follow-up or clarification questions they want to ask. After the parent mastered reinforcement technique and data had been collected, then Model-Lead-Test training started. A reasonable timeline for this initial stage was last approximately one week. When MLT training and data collection were completed, Lesson 2 begins. Lesson 2 included the concept of Prompt Hierarchy and a 3-step prompt procedure was taught (see Appendix E). The parent must achieve mastery of prompting performance, $80 \%$ correct response before starting the next phase. In lesson 3 parents were taught (Oral Lecture/Discussion and/or MLT) how and when to fade prompts and given examples of when to implement them (See Appendix F). The Oral Lecture/Discussion for Lesson 3 was skipped if MLT training had previously been demonstrated to be the most effective during lesson 1 and 2 . That was, the order of parent training phases were determined by the impact of Oral Lecture/Discussion and MLT on the parent's use of reinforcement. If MLT produced the same or better results than Oral Lecture/Discussion, it would be used first for the training of the use of prompting and Oral Lecture/Discussion would be used second with the approach with the best overall impact used for the training on the use of fading. Maintenance phases would be the last phase for all techniques to assess the durability of the effects observed. On the contrary, if Oral Lecture/Discussion training produced better results than MLT, the opposite sequences (Baseline $\rightarrow$ Oral Lecture/Discussion $\rightarrow$ MLT $\rightarrow$ Maintenance) would be implemented. In other words, depending on the patterns in the results of the reinforcement and prompting training that showed which training procedures produce the greater effects that would be the one replicated in the training for fading by the primary researcher. The sequence of training 
procedures for Parents B and C would be determined in the same way based on the results produced by each training procedure.

During the Model-Lead-Test phase, each parent observed the primary researcher conducting the reinforcement, prompt, and fading procedures sequentially with the target learner, and received in vivo Model-Lead-Test training. Each parent will participate in 3-5 training sessions until they achieve mastery. Therefore, each parent had to master each lesson in sequence and they all had independent staggered schedules. In other words, each parent learned through the Model-Lead-Test training procedure and demonstrate that he/she could correctly deliver reinforcers with at least $80 \%$ accuracy before continuing to OLD or maintenance on reinforcement and lesson 2, Prompting and correctly deliver prompts with at least $80 \%$ accuracy before continuing to OLD or maintenance and lesson 3 , Fading and correctly fade prompts with at least $80 \%$ accuracy before continuing to OLD or maintenance.

In the Modeling period, the parent focused on observing the researcher for 3 to 5 trials. In the Leading period, the parent applied the techniques with the researcher. For example, in the reinforcement session, the researcher provided behavior specific praise and reinforcers to the learner participant if the parent did not provide those in the right timing. If the parent participant failed to achieve $80 \%$ accuracy or misses more than 3 consecutive opportunities, the researcher would go back to demonstrate the technique for 3 trials (a repeat of the Modeling) then had the parent participant to go through the Leading session again. After successful completion of the Leading session, the Testing session started. The parent participant demonstrated the technique on his/her own. Accuracy of MLT implementation for parent participant checklist was applied to review the learning outcomes (See Appendix P, Q, $\& \mathrm{R})$.

During the maintenance phase, the researcher continued to video record and coded within a given amount of time for the correct response per opportunity for using reinforcers, 
prompting, and fading. The researcher also recorded the outcomes of child-participant's talker, participator, and problem solver repertoires. In this phase, the researcher did not provided any strategies (ABA Oral Lecture/Discussion or Model-Lead-Test) or feedback. Researcher collected data once a week for a month to track the durability of the results.

As a secondary measure, the outcomes of talker, participator, and problem solver repertoires of the child learner per 15 minutes for each repertoire were assessed, based on the modified CLM Curriculum. For example, CLM Lesson 11.2 Repertoire, Talker 0.001 (mand>echoic): Repeating words for preferred items or T actions without item displayed (Tucci, 2006). Due to learners' different abilities, the criterion and content of the learning materials varied or were modified. If the learner was verbal and able to say sentences but the enunciation was not clear, then the criterion was changed from repeating words to repeating sentences. This data were recorded and collected from the same video recording of the session.

\section{Data Collection and Analysis}

\section{Data Collection Procedures}

The primary researcher videotaped all behavior observations. The videotapes were then observed and coded by the primary researcher, as well as the second and third observers who were both blind to the conditions. Second and third observers attended a one-hour training session prior to the start of the study. The behavior was measured using a response per opportunity recording system. Each time the target behavior appeared counts as one opportunity. If the parent responded correctly based on the protocol then researcher recorded "yes, independent" was recorded. And, if the parent responded correctly after prompting, the researcher recorded "yes, with prompt". If after two prompts from researcher, the parent still did not respond correctly, then "No" was recorded in the checklist. Point by point measure was applied in the data collection procedure. All data were collected from direct observations 
with the exception of the parents' social validation responses regarding their experience, which were collected via a questionnaire.

During the interaction recording session, the researcher set up the video recorder on the table of the playroom. The researcher positioned her self in the corner so that she could record and observe the participants at the same time but minimally distracted the participants. By observing the video, a data sheet (see Appendix J, K, \& L) was used to record all instances of a parent participant properly using reinforcement, prompting, and fading when the opportunity occurred. In the same datasheet all responses by the child participant were also collected (see Appendix M, N, \& O). When the researcher began to collect data, the exact time was recorded, and the end time was recorded as soon as the data collection was terminated. Data collection started when the timer was on and lasts for 15 minutes. When the timer was off, data collection was ended and video was turned off too. The 15-minute intervals were selected because parents were usually more successful for the first 15 minutes when they were interacting with their learners.

\section{Inter Observer Agreement}

Inter observer agreement was used for checking inter-rater reliability. The point-bypoint agreement method was applied to assess the agreement between two observers. The primary and secondary observer inter-rater reliability was for parent and learner participation. The primary and third observer inter-reliability was used to establish whether the researcher was correctly demonstrating OLD and MLT training protocols. Therefore, the observers marked occur or not occur for the target behavior during each discrete opportunity. In other words, the observers followed the checklist created by primary researcher to record the outcomes of the training for parent participants and child participants (See Appendix $\mathrm{J}$ to $\mathrm{R}$ ). If the participant responded correctly with every opportunity and as well as correct responses, 
the observer marked a plus. Alternatively, a minus was marked for incorrect response for each opportunity.

The three observers, primary, second, and third observers practiced coding observational data until they reached at least $80 \%$ inter-rater reliability. An 80 percent interrater reliability was the minimum threshold amount at which the ABA field generally considers sufficient for publication. The primary researcher reviewed all of the videos and record the results. For at least $33 \%$ of the behavior observation sessions, both primary and second or third observers collected behavioral data to determine interobserver agreement. Percent of agreement was calculated by dividing the number of observational agreements by the number of agreements plus number of disagreements $(\mathrm{A} /[\mathrm{A}+\mathrm{D}])$ for each session and multiplying by $100 \%$. For data to be considered valid, it was generally held to an $80 \%$ or higher standard. The researcher calculated the interobserver agreement. In this study, Kappa Statistic was also calculated to assess the level of interobserver agreement when statistically corrected for chance agreements. If the observers are in complete agreement then $k=1$. A kappa of 0 indicates agreement is equivalent to chance. If $\mathrm{k}<0$ indicates less than chance agreement; 0.01-0.20 means slight agreement; 0.21-0.20 indicates fair agreement; 0.41-0.60 means moderate agreement; 0.61-0.80 means substantial agreement; 0.81-0.99 means almost perfect agreement (Viera, \& Garrett, 2005).

\section{Social Validity}

Social validity data were collected in order to assess how meaningful the training was for the parents and what they had learned in the training and how satisfied they were with the training procedures, goals, and the social importance of the effects (see Appendix B \& C). The social validity measure was adapted from Lindsey (2009). At the last home visit, parents were given the questionnaire by the researcher to complete without the primary researcher present. The social validity measure was scored by assigning numbers (one for strongly 
disagree and five for strongly agree) to each response. The means for each parent's answers were calculated later. The answers to the social validity questionnaire were answered on a standard 5 point Likert scale and displayed in the result section.

\section{Observer Behavior}

Data were collected on observed parent and child behavior. Response per opportunity data were recorded for the following parent behaviors: properly implementing reinforcement, prompting, and fading techniques. Response per opportunity data were collected for the following child behaviors: independently responding to directives from parents, prompted participation, and correct CLM talker, participator, and problem solver responses (see Appendix $\mathrm{M}$ to O). For talker repertoire, parent participant followed protocol to ask the learner to repeat sentences or answer questions. The researcher only redirected the parent if the parent falled too far from protocols to prevent skewing outcomes. (i.e. off topic, etc.) For example, CLM Lesson 11.2, Talker 0.001: Repeating sentences for preferred items or teacher actions without item displayed. The observers marked plus if the learner responded correctly to read sentences or answer questions. For participator repertoire, parent participant followed protocol asking the learners to participate in activities. For example, CLM Lesson 8, Participator 0.501: Performs 2 sets of five consecutive responses. Observers circled one opportunity each time the parent participant asked the learner to engage in activities and noted what types of prompts the parent provides when the learner failed to respond within 3 seconds. Only two prompts were provided in the research design. For problem solver repertoire, parent participant identified the current types of prompt, called initial prompt, she/he was applying with the selected skill in this study. The skill had to be pretty close to mastery by learner. For example, CLM Lesson 12, Problem Solver 0.801: Solves problem by manipulation of a variety of different sized and shaped parts to fit them in place or remove them from a place; completes 2 sets of five pieces of each. Observers circled one opportunity 
when every time the parent attempted to prompt the learner and recorded the types of prompts. The result was compared to the initial prompt. If the prompts provided by parent during each step were less intrusive than the initial prompts, some observers might consider that fading was occurring in their analysis of the data. Each variable was analyzed separately. Changes in behavioral observation measures of each parent/child dyad were graphed using a multiple baseline design and visual inspection was implemented to conclude the outcomes. The impact of the intervention was checked through the changes of means, levels, trends, and variability. Data were analyzed by calculating changes in means. Changes in level also were visually investigated by observing the shift of performance from the end of one phase to the beginning of the next phase. The split-middle technique was applied to examine the changes in trend, to assess whether the intervention is associated with a change in performance. Variability in data showed how consistently the intervention changes behavior from session to session and phase to phase. Usually the greater the variability in the data, the more data points that were required to obtain stability (Kennedy, 2005).

In addition, the result of Model-Lead-Test in the reinforcement phase was analyzed immediately to determine whether Oral Lecture was skipped or implemented later. Therefore, the possibilities of this study design might look as below (Table 5) if Model-Lead-Test showed the greater positive impact in participants' performance (OLD stands for oral lecture discussion phase; MLT stands for model-lead-test phase). 
Table 5

The possibilities of study design (+ means greater degree of impact)

\begin{tabular}{|l|l|l|l|l|}
\hline & Baseline & Phase 1 & Phase 2 & Maintenance \\
\hline Parent A & & & & \\
\hline Reinforcement & & OLD & MLT + & \\
\hline Prompting & & MLT + & OLD & \\
\hline Fading & & MLT & MLT & \\
\hline Parent B & & & & \\
\hline Reinforcement & & MLT + & OLD & \\
\hline Prompting & & OLD & MLT + & \\
\hline Fading & & MLT & MLT & \\
\hline Parent C & & & & \\
\hline Reinforcement & & MLT & MLT & \\
\hline Prompting & & MLT & MLT & \\
\hline Fading & & MLT & MLT & \\
\hline
\end{tabular}




\section{Chapter Three}

\section{Results}

\section{Overview}

The results of this study are addressed below. First, inter-observer agreement data are reported. Next, results are reported for relevant variables for each of the following research questions: 1) Is parent training that uses a Model-Lead-Test procedure effective in increasing parents' appropriate use of reinforcement, prompting, and fading techniques? 2) As the parents appropriately use reinforcement, prompting, and fading techniques, do the learners' behavioral repertoires for "talker, participator, and problem solver" improve as well? For each research question, the data for mean, level, trend, and variability are graphically depicted below. Finally, the results of social validity for both OLD and MLT trainings will be reported as well.

\section{Interobserver Agreement}

Percent of agreement is calculated by dividing the number of agreements by the number of agreements plus disagreements $(\mathrm{A} /[\mathrm{A}+\mathrm{D}])$ for each session and multiplying by 100\%. The formula for Kappa statistic is $\operatorname{Pr}(\mathrm{a})-\operatorname{Pr}(\mathrm{e}) / 1-\operatorname{Pr}(\mathrm{e}) \cdot \operatorname{Pr}(\mathrm{a})$ is the observed percentage agreement. $\operatorname{Pr}(\mathrm{e})$ is the probability of random agreement.

Inter-rater reliability data were calculated for $33 \%$ of sessions in each phase for each dyad. The response per opportunity data were considered reliable if the mean percent of agreement for each dyad was at or above $80 \%$ (Kazdin, 2011).

The researcher randomly selected $33 \%$ of the video recorded sessions to be independently reviewed by a second observer (18 of 56 sessions for Mark; 17 of 51 sessions for Brandy; 12 of 38 session for Jenny). The second observer similarly evaluated the sessions and a percent of agreement between the primary and secondary observer was calculated. The researcher calculated the percent of agreement and Kappa for each session, then determined 
the average number between those agreements. The mean percentages of agreement for Mark and Chuck were $88.9 \%$, Brandy and Albert were 95.6\%, and Jenny and Mike were 95\%. The mean of Kappa for Mark was 0.73 (13.09 divided by 18); Brandy was 0.81 (13.807 divided by 17$)$; and Jenny was 0.86 (9.50 divided by 12$)$.

The third observer scored "Treatment Fidelity", which included the Effective OLD and MLT implementation Integrity observation checklists (4 of 13 training sessions) and the parent attainment, which include the OLD and MLT training interobserver agreement sheets (4 of 13 training sessions). Based on an independent review of $33 \%$ of random samples of intervention sessions, there was a $95 \%$ agreement for researcher fidelity and an $88.8 \%$ parent attainment of objectives.

\section{Research Questions}

\section{Is parent training using a Model-Lead-Test procedure effective in increasing parents' appropriate use of reinforcement, prompting, and fading techniques?}

To investigate these questions, parents were observed interacting with their children during 10-15 minutes video taped sessions. Results for the dependent variables for each phase are described below.

During the data collection, the following dependent variables for parent behaviors were recorded and coded: proper use of reinforcement, prompting and fading. The responses "per opportunity" for each behavior was recorded.

\section{Enrollment}

Six parents were screened for participation in the study. Two families declined after screening and one child participant did not meet the criteria for the study. Three parents (Mark, Brandy, \& Jenny) and their children (Chuck, Albert, \& Mike) completed the full data collection trials, which contained baseline, training (OLD and/or MLT), and maintenance. These three dyads were chosen based on the aforementioned criteria. 


\section{Demographic Data}

Tables 6, and 7 summarize demographic, and educational information for the three participating parent-child dyads. As shown in Table 6, there were three parent participants, 2 females and 1 male ages varying between 35 and 43. Their education levels ranged from high school to college and all of them speak English. As shown in Table 7, the three child participants were all boys; all of them were diagnosed with Autism Spectrum Disorder and placed in special education classroom and their ages were from 8 to 10 years old. The severity of their disabilities would be characterized as moderate to severe. Child participants, Chuck and Mike, display maladaptive behavior in the form of injurious to others. In addition, Chuck is non-verbal and has very limited skills for self-care and hygiene and is self-injurious. Albert and Max display comprehension deficits and lack participation skills.

Table 6

Demographic data for the three parent participants

\begin{tabular}{lllll}
\hline & Age & Education & Gender & Ethnicity \\
\hline $\begin{array}{l}\text { Participant A } \\
\text { Mark }\end{array}$ & 43 & College & Male & Caucasian \\
$\begin{array}{l}\text { Participant B } \\
\text { Brandy }\end{array}$ & 35 & $\begin{array}{l}\text { Associate of } \\
\text { Arts Degree }\end{array}$ & Female & Latino/Hispanic \\
$\begin{array}{l}\text { Participant C } \\
\text { Jenny }\end{array}$ & 36 & $\begin{array}{l}\text { High school } \\
\text { Highole }\end{array}$ & Female & Latino/Hispanic \\
\hline
\end{tabular}

Table 7

Demographic data for the three child participants

\begin{tabular}{lllll}
\hline & Age & Education & Gender & Diagnosis \\
\hline $\begin{array}{l}\text { Participant A' } \\
\begin{array}{l}\text { Chuck } \\
\text { Participant B' }\end{array}\end{array}$ & 10 & Special Ed & Boy & Autism \\
$\begin{array}{l}\text { Albert } \\
\text { Participant C' } \\
\text { Mike }\end{array}$ & 10 & Special Ed & Boy & Autism \\
\hline
\end{tabular}




\section{Description and Analysis of Data}

Parental learning of the program was evaluated by computing parent adherence to session objectives (as scored by independent observers) and ratings of parent satisfaction.

The effect of the parent training program on the children's behaviors (talker; participator; problem solver/operate) was compared with repeated measures in different phases, including data from Baseline, OLD, MLT and Maintenance, in the study. To analyze changes in the use of proper reinforcement/prompting/fading, the total number of correct responses of using proper reinforcement/prompting/ fading were divided by the total number of opportunities to apply reinforcement/prompting/fading while interacting with their children and were converted to a percentage of properly applying reinforcement/prompting/fading.

In reinforcement, the procedure in the study consisted of having the parent read material to the learner. Then the parent requested the learner to answer questions about what was just read or repeat sentences recently heard to shape their "talker" repertoire. The data represents the videotaped session of the parent and learner interaction and the researchers' scoring of parental responses, both appropriate (specific) and inappropriate (i.e. parent exhibits no response, limits response to only saying "good job", etc.) implementation of reinforcement to the learners' correct responses. In prompting, the procedure in the study consisted of having the parent instruct the learner. Then the parent prompted the learner to respond correctly to shape their "participator" repertoire. The data represents the videotaped session of the parent and learner interaction and the researchers' scoring of parental responses, both appropriate (proceeding least to most or errorless prompts) and inappropriate (i.e. parent exhibits telling the child to listen, continuing to use the same type of the prompt or using "nono" prompt) implementation of prompting to the learners' incorrect responses. In fading, the procedure in our study consisted of having the parent gradually fade their prompts to shape 
the child participants' "problem solver" repertoire, which was identified and observed by each parent to be the most difficult task for her/his child participant. Teeth brushing, morning routine, and dish washing were problematic self-care tasks identified by the three parent participants. Those self-care tasks were considered by parent participants to be causing many of the maladaptive behaviors of their learners and required a lot of help from adults. The data represents the videotaped session of the parent and learner interaction and the researchers' scoring of parental responses, both appropriate (fading the full verbal or physical prompt) and inappropriate (i.e. parent exhibits full physical or verbal prompts, etc.) implementation of fading to the learners' independent behaviors.

The child participant behaviors for "talker/participator/problem-solver" were measured by recording the correct response per opportunity. To analyze the changes in the talker/participator/and problem-solver repertoires, the total number of correct responses for talker/participator/ and problem solver were divided by the total number of opportunities to apply talker/participator and problem solver while interacting with their parents and were converted to a percentage of correct response for each repertoire.

\section{Mark's Proper Implementation of Reinforcement, Prompting \& Fading}

Table 8 displays the mean, range and levels of Mark's proper implementation of reinforcement, prompting and fading for each phase of interaction with his learner. The table shows the mean percent followed by the percent range in parentheses and level with italics. Mark's case illustrates an increase in the percentages of applying reinforcement during observations. The means reported were respectively 5\%, 9.25\%, 96.67\%, and 97.5\%, respectively, for baseline, OLD, MLT and maintenance phases. For prompting session, the means were $4.29 \%, 88.75 \%, 87.76 \%$ and $97.5 \%$. There was a slight decrease from MLT phase $(88.75 \%)$ to OLD phase $(87.76 \%)$ During the fading session, Mark showed an overall increase in the means for $2.5 \%, 92.5 \%$, and $96 \%$. The data across phases were displayed by 
changes in level, especially the dramatic change when MLT training was introduced in each reinforcement, prompting and fading sessions. There was a decreasing level from MLT $(87.50 \%)$ to OLD $(80 \%)$ phase in prompting sessions. The OLD training was not implemented in the fading training because MLT showed significantly better results in reinforcement and prompting session. 
Table 8

Means, Ranges and Levels of Mark's Percentage of Implementing Proper Reinforcement, Prompting \& Fading

\begin{tabular}{lcccc}
\hline $\begin{array}{l}\text { Parent } \\
\text { Mark }\end{array}$ & $\begin{array}{c}\text { Phase I } \\
\text { (Baseline) }\end{array}$ & $\begin{array}{c}\text { Phase II } \\
\text { (Training OLD/MLT) }\end{array}$ & $\begin{array}{c}\text { Phase III } \\
\text { (Training MLT/OLD) }\end{array}$ & $\begin{array}{c}\text { Phase IV } \\
\text { (Maintenance) }\end{array}$ \\
\hline Reinforcement & $5 \%$ & $9.25 \%$ OLD & $96.67 \%$ MLT & $97.5 \%$ \\
& $(0-20)$ & $(0-20)$ & $(90-100)$ & $(87.5-100)$ \\
Prompting & $0 \%$ & $20 \%, 0 \%$ & $100 \%, 100 \%$ & $90 \%$ \\
& $4.29 \%$ & $88.75 \%$ MLT & $87.76 \%$ OLD & $97.50 \%$ \\
Fading & $(0-0)$ & $(80-100)$ & $(80-100)$ & $(90-100)$ \\
& $0 \%$ & $80 \%, 87.50 \%$ & $80 \%, 83.30 \%$ & $100 \%$ \\
& $2.5 \%$ & N/A & $92.50 \%$ MLT & $96 \%$ \\
& $(0-10)$ & & $(90-100)$ & $(90-100)$ \\
& $0 \%$ & & $90 \%, 90 \%$ & $100 \%$ \\
\hline
\end{tabular}

Figure 1 is a multiple baseline illustrating the results of Mark. It also demonstrates that his performance improved substantially after each training, OLD and MLT, as well as the maintenance phase. The split-middle technique was applied to analyze the trend. Visual inspection reveals an upward trend and an increasing level in the training and maintenance phases across reinforcement, prompting and fading sessions. The overall variability for Mark's sessions is minimal. The results of standard deviation for all phases are between 0.04 to 0.1 . 


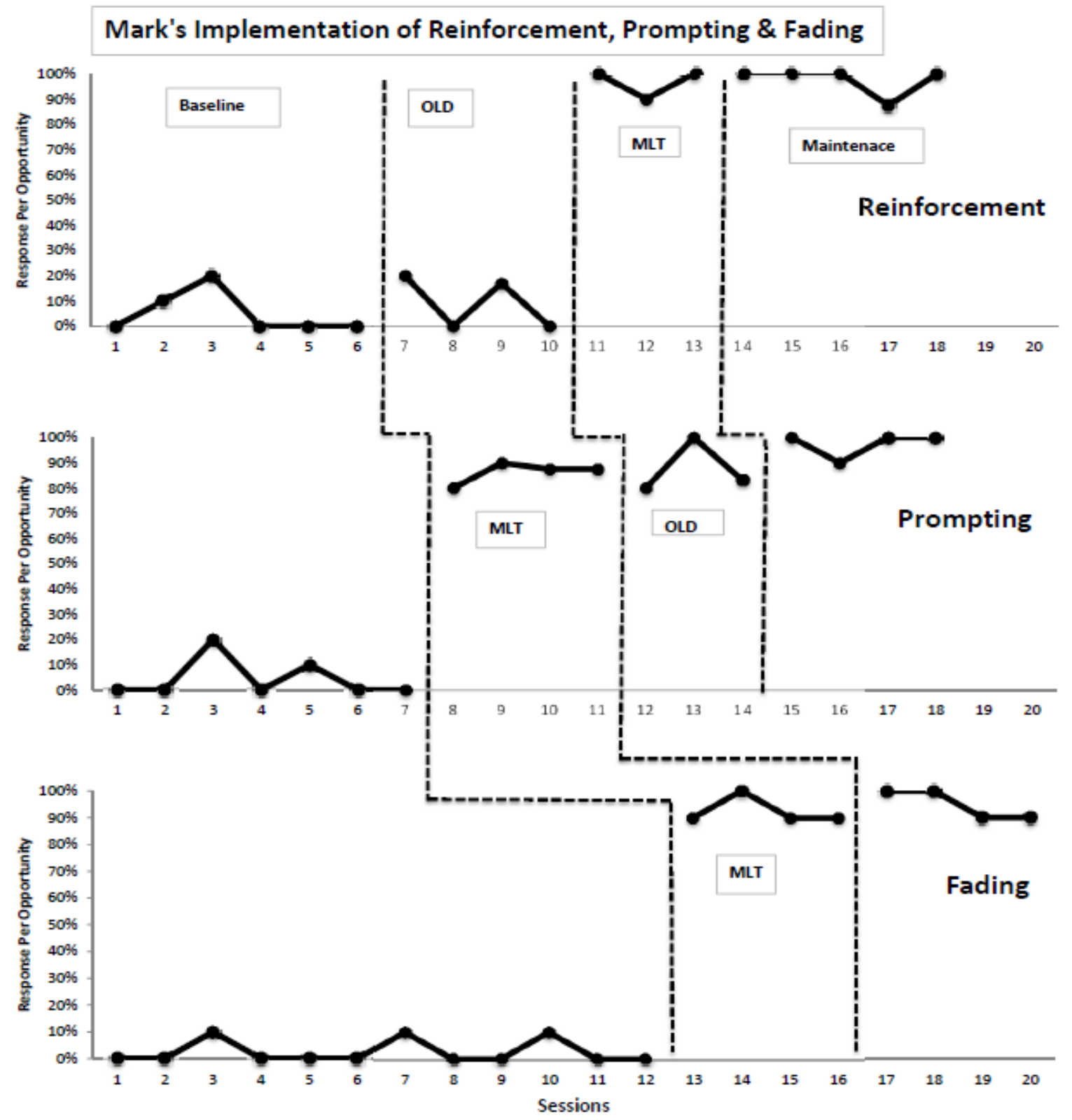

Figure 1. Mark's percentage of proper implementation of reinforcement, prompting $\&$ fading. 


\section{Brandy's Proper Implementation of Reinforcement, Prompting \& Fading}

Table 9 displays the means and ranges of Brandy's proper implementation of Reinforcement, Prompting and Fading techniques. The table describes the mean percentages followed by the ranges in parentheses and levels with italic. In the reinforcement section, Brandy increased her mean percentages of applying reinforcement separately from $0 \%, 85 \%$, $91.1 \%$ to $96.88 \%$. In the prompting section, the mean percentages of Brandy's implementing prompting were $4.5 \%, 57.13 \%, 100 \%$ and $97.50 \%$. During the fading section, the mean percentages were $3.64 \%, 95 \%$, and $97.50 \%$. The results for this study as indicated in table 11 show that when compared to baseline measures, Brandy's overall performance during OLD, MLT and Maintenance phases increased the means and levels after receiving training from the researcher. The OLD training was not implemented in the fading section because the results during reinforcement and prompting showed a greater impact from MLT training. Overall, Brandy increased the percentages of using reinforcement, prompting, and fading after both OLD and MLT trainings.

Table 9

Means, Ranges and levels of Brandy's Percentage of Implementing Proper Reinforcement, Prompting \& Fading

\begin{tabular}{lcccc}
\hline $\begin{array}{l}\text { Parent } \\
\text { Brandy }\end{array}$ & $\begin{array}{c}\text { Phase A } \\
\text { (Baseline) }\end{array}$ & $\begin{array}{c}\text { Phase B/C } \\
\text { (Training OLD/MLT) }\end{array}$ & $\begin{array}{c}\text { Phase B/C } \\
\text { (Training MLT/OLD) }\end{array}$ & $\begin{array}{c}\text { Phase D } \\
\text { (Maintenance) }\end{array}$ \\
\hline Reinforcemen & $0 \%$ & $85 \%$ MLT & $91.1 \%$ OLD & $96.88 \%$ \\
$\mathrm{t}$ & $(0-0)$ & $(60-100)$ & $(84.8-100)$ & $(87.5-100)$ \\
Prompting & $0 \%$ & $60 \%, 100 \%$ & $90 \%, 90 \%$ & $100 \%$ \\
& $4.5 \%$ & $57.13 \%$ OLD & $100 \%$ MLT & $97.5 \%$ \\
& $(0-12.5)$ & $(50-71.4)$ & $(100-100)$ & $(90-100)$ \\
Fading & $0 \%$ & $70 \%, 50 \%$ & $100 \%, 100 \%$ & $100 \%$ \\
& $3.64 \%$ & N/A & $95 \% \mathrm{MLT}$ & $97.50 \%$ \\
& $(0-10)$ & & $(90-100)$ & $(90-100)$ \\
& $0 \%$ & & $90 \%, 100 \%$ & $90 \%$ \\
\hline
\end{tabular}

Figure 2 below is a multiple baseline illustrating the outcomes for Brandy and data are graphically displayed. The sessions in which reinforcement, prompting, and fading were 
introduced through OLD and MLT are recorded in the graph. Visual inspection of the graph shows a mild variability in the data, and an increase in both trend and level for OLD and MLT training. The data across phases were displayed by shifts in level and an especially significant change is seen when MLT training was introduced for Brandy. The significant change is highlighted by the fact that the reinforcement phase started at $60 \%$. Alternately, the variability for Brandy in each phase is minimal and the standard deviations are between 0.04 to 0.2 .

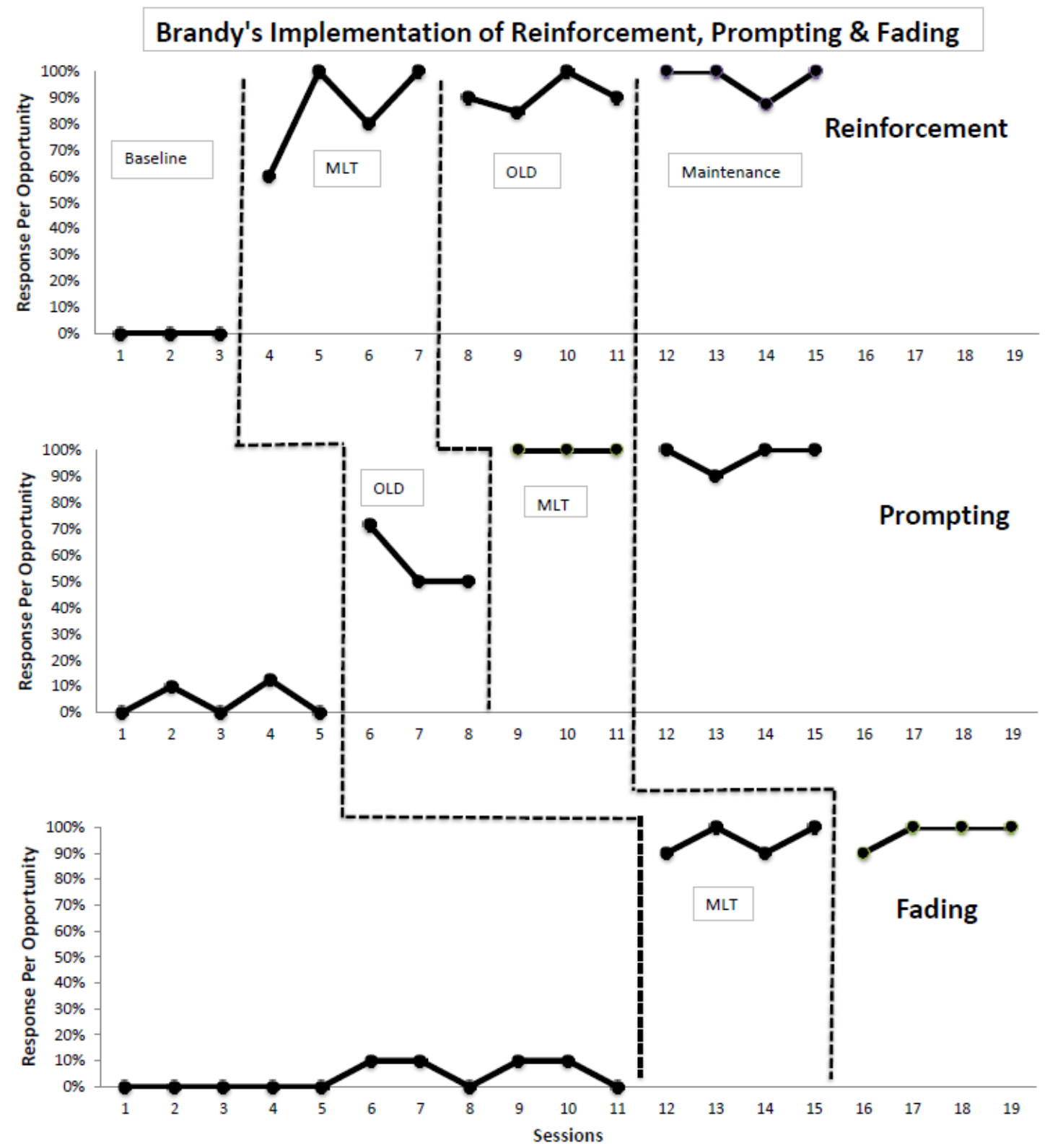


Figure 2. Brandy's percentage of proper implementation of reinforcement, prompting \& fading. 


\section{Jenny's Proper Implementation of Reinforcement, Prompting \& Fading}

Table 10 displays the mean, range and levels of Jenny's proper implementation of Reinforcement, Prompting and Fading for each phase of interaction with her learner. The table shows the mean percent followed by the percent range in parentheses and level in italics. Jenny showed overall increases in the percent of applying reinforcement during observations. The means respectively were $0 \%, 96.67 \%$, and $97.5 \%$ for baseline, OLD, MLT and maintenance phases. For prompting session, the means were $6 \%, 100 \%$, and $95 \%$. There was a slight decrease from MLT phase (100\%) to maintenance phase (95\%). Jenny increased her proper use of fading from baseline $(5.71 \%)$ to prompting $(92.5 \%)$ but decreased from prompting $(92.5 \%)$ to maintenance $(85 \%)$ due to the low percent in the first performance $(40 \%)$ in maintenance phase. The data across phases were displayed by shifts in level, especially the dramatic change when MLT training was introduced for Jenny. The OLD training was not implemented in Jenny's sessions because MLT showed significantly better results in reinforcement and prompting sessions for the previous two participants, Mark and Brandy.

Table 10

Means, Ranges and Levels of Jenny's Percent of Implementing Proper Reinforcement, Prompting \& Fading

\begin{tabular}{lcccc}
\hline $\begin{array}{l}\text { Parent } \\
\text { Jenny }\end{array}$ & $\begin{array}{c}\text { Phase A } \\
\text { (Baseline) }\end{array}$ & $\begin{array}{c}\text { Phase B } \\
\text { (Training OLD/MLT) }\end{array}$ & $\begin{array}{c}\text { Phase C } \\
\text { (Training MLT/OLD) }\end{array}$ & $\begin{array}{c}\text { Phase D } \\
\text { (Maintenance) }\end{array}$ \\
\hline Reinforcement & $0 \%$ & N/A & $96.76 \%$ MLT & $98 \%$ \\
& $(0-0)$ & & $(90-100)$ & $(90-100)$ \\
Prompting & $0 \%$ & & $100 \%, 100 \%$ & $90 \%$ \\
& $6 \%$ & N/A & $100 \% \mathrm{MLT}$ & $95 \%$ \\
Fading & $(0-30)$ & & $(100-100)$ & $(90-100)$ \\
& $0 \%$ & & $100 \%, 100 \%$ & $90 \%$ \\
& $5.71 \%$ & N/A & $92.5 \% \mathrm{MLT}$ & $85 \%$ \\
& $(0-10)$ & & $(90-100)$ & $(90-100)$ \\
& $10 \%$ & & $80 \%, 100 \%$ & $40 \%$ \\
\hline
\end{tabular}


Figure 3 below is a multiple baseline illustrating the performance of Jenny, and shows a continuous improvement after MLT was introduced and continuing through the maintenance phase. The researcher started the training with Jenny, after analyzing the outcomes of Participant A, Mark, and Participant B, Brandy, which showed that Model-LeadTest training had greater impacts on both of their performance scores for implementing reinforcement and prompting.

Figure 3 graphically displays the data. The sessions in which reinforcement, prompting, and fading were introduced through MLT are noted in the graph. Visual inspection reveals an increasing trend and level in the training and maintenance phases. Initially, the data during fading sessions showed $40 \%$ in the beginning of maintenance but increased to $100 \%$ for the remaining 3 weeks. Jenny's data displays a starting low of $40 \%$ and thereafter a more steady variability. The standard deviations are between 0 to 0.25 .

The results for this study as indicated by Figure 3 show that when compared to baseline measures, Jenny's performance during MLT and Maintenance phases increased for all variables after receiving training from the researcher. 


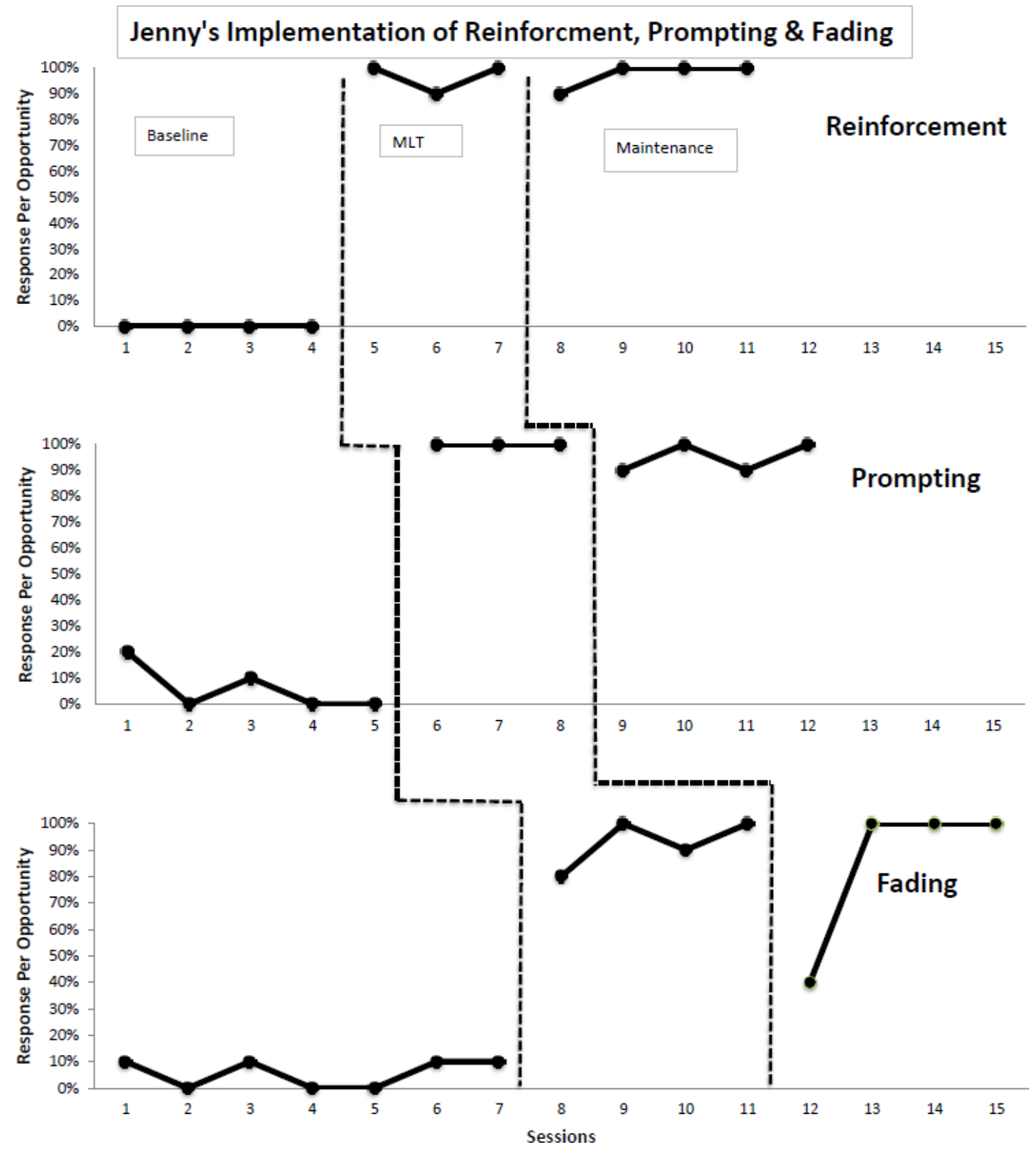

Figure 3. Jenny's percentage of proper implementation of reinforcement, prompting \& fading.

Do the learner's talker, participator, and problem solver repertoires improve for learners with Autism Spectrum Disorder as their parents increase appropriate use of reinforcement, prompting, and fading techniques?

To answer these questions, learners were observed completing 10-15 minutes of interactions with their parents. The results for each dependent variable for each repertoire are described below. 


\section{Child Participants' Performance in Talker Repertoire}

Table 11 displays the mean percentages of the observed "talker" repertoire for each child participant across different phases. All of the three child participants, Chuck, Albert, and Mike showed an increase in their talker repertoires. Chuck increased his mean percentiles from $36.67 \%, 42.5 \%, 63.33 \%$ to $80 \%$. Albert showed increasing from baseline $(60 \%)$ to MLT (72.5\%) and maintenance (90\%) phases but slightly decreased from MLT (72.5\%) to OLD (67.50\%). Mike overall increased his talker repertoire through baseline (67.5\%), MLT $(86.67 \%)$ to maintenance $(92.50 \%)$.

Table 11

Means and Ranges of Children's Percentage of Talker Repertoire

\begin{tabular}{lcccc}
\hline Child & $\begin{array}{c}\text { Phase A } \\
\text { (Baseline) }\end{array}$ & $\begin{array}{c}\text { Phase B } \\
\text { (Training OLD/MLT) }\end{array}$ & $\begin{array}{c}\text { Phase C } \\
\text { (Training MLT/OLD) }\end{array}$ & $\begin{array}{c}\text { Phase D } \\
\text { (Maintenance) }\end{array}$ \\
\hline Chuck & $36.67 \%$ & $42.5 \%$ OLD & $63.33 \%$ MLT & $80 \%$ \\
& $(20-50)$ & $(20-40)$ & $(60-70)$ & $(60-100)$ \\
Albert & $50 \%$ & $20 \%, 60 \%$ & $60 \%, 60 \%$ & $70 \%$ \\
& $60 \%$ & $72.5 \% \mathrm{MLT}$ & $67.50 \%$ OLD & $90 \%$ \\
& $(50-70)$ & $(50-100)$ & $(60-70)$ & $(60-100)$ \\
Mike & $70 \%$ & $50 \%, 90 \%$ & $70 \%, 70 \%$ & $100 \%$ \\
& $67.5 \%$ & $\mathrm{~N} / \mathrm{A}$ & $86.67 \% \mathrm{MLT}$ & $92.50 \%$ \\
& $(60-70)$ & & $(70-90)$ & $(80-100)$ \\
\hline
\end{tabular}

Figure 4 below is a bar graph illustrating the means of child participants' talker repertoire during the study in each phase when the parent participants were implementing reinforcement techniques with them. Overall, the results of three child participants' talker repertoire increased, with the exception of Albert. For Albert, there was a decrease from phase B/MLT (72.50 to 67.50) to phase C/OLD. 


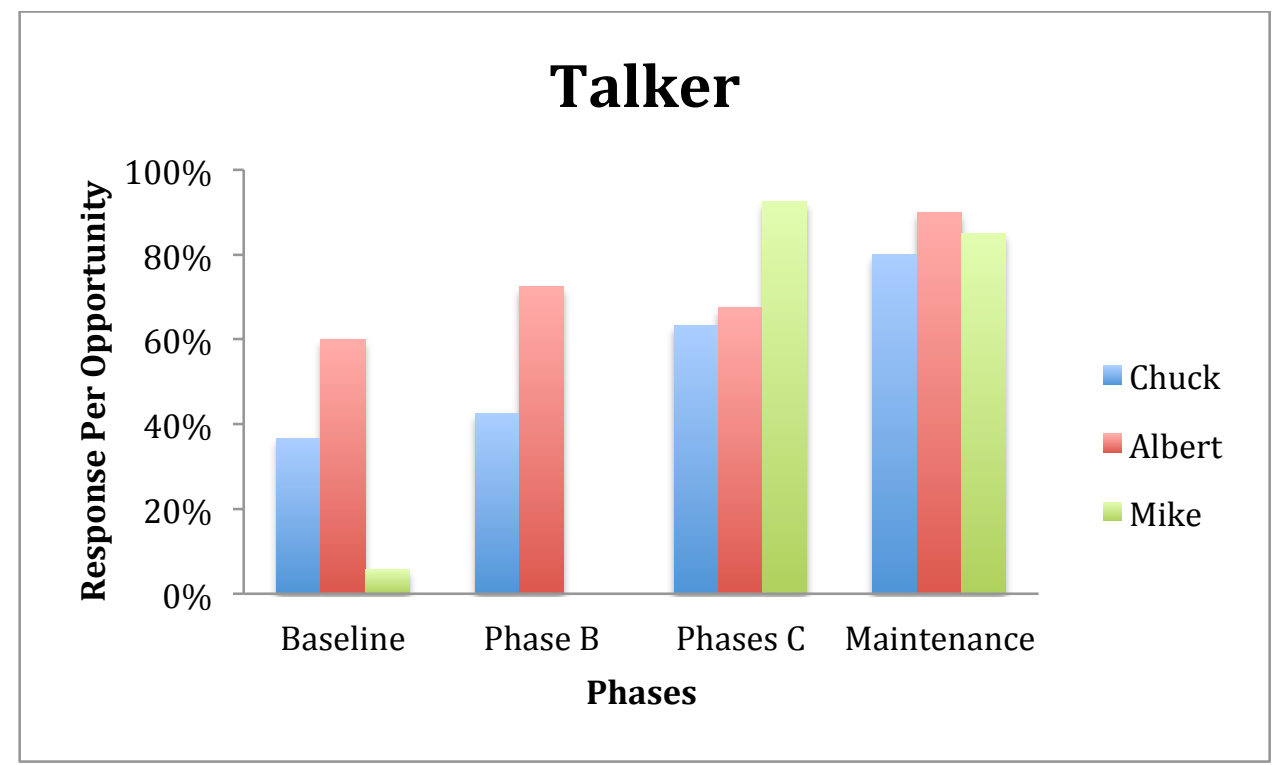

Figure 4. The Means for Child Participants' Talker repertoire

\section{Child Participants' Performance in Participator Repertoire}

Table 12 displays the means and ranges of the child participants' percentages of "participator" repertoire during observations. The table displays the mean percentages followed by the ranges in parentheses. Overall, Chuck increased his percentage of responding to his dad's directions. The means were $19.69 \%, 77.75 \%, 73.33 \%$ and $92.59 \%$. Albert increased his overall participator repertoire from $14 \%, 46.67,88.33 \%$ to $87.50 \%$ through baseline, OLD, MLT and maintenance sessions. Mike showed an increase from baseline $(50 \%)$ to $\operatorname{MLT}(83.33 \%)$ but a decrease from MLT $(83.33 \%)$ to maintenance $(80 \%)$.

Table 12

Means and Ranges of Children's Percent of Participator Repertoire

\begin{tabular}{lcccc}
\hline Child & $\begin{array}{c}\text { Phase A } \\
\text { (Baseline) }\end{array}$ & $\begin{array}{c}\text { Phase B } \\
\text { (Training OLD/MLT) }\end{array}$ & $\begin{array}{c}\text { Phase C } \\
\text { (Training MLT/OLD) }\end{array}$ & $\begin{array}{c}\text { Phase D } \\
\text { (Maintenance) }\end{array}$ \\
\hline Chuck & $19.69 \%$ & $77.50 \%$ MLT & $73.33 \%$ OLD & $92.50 \%$ \\
& $(0-62.5)$ & $(70-90)$ & $(70-80)$ & $(90-100)$ \\
Albert & $25 \%$ & $70 \%, 90 \%$ & $70 \%, 70 \%$ & $90 \%$ \\
& $14 \%$ & $46.67 \%$ OLD & $83.33 \% \mathrm{MLT}$ & $87.50 \%$ \\
& $(0-30)$ & $(40-50)$ & $(80-90)$ & $(80-90)$ \\
Mike & $30 \%$ & $70 \%, 100 \%$ & $100 \%, 100 \%$ & $100 \%$ \\
& $50 \%$ & $\mathrm{~N} / \mathrm{A}$ & $83.33 \% \mathrm{MLT}$ & $80 \%$ \\
& $(20-40)$ & & $(80-90)$ & $(80-90)$ \\
\hline
\end{tabular}


Figure 5 below is a bar graph illustrating the means of child participants' participator repertoires during the study in each phase when the parent participants were implementing prompting techniques with them. Albert showed improvement in his "participator" repertoire across baseline through OLD, MLT and maintenance. Both Chuck and Mike showed a decrease in data during prompting procedure. The result declined from $77.50 \%$ to $73.33 \%$ from MLT to OLD phase for Chuck. Mike had a decrease of from 83.33 to $80 \%$ from MLT to maintenance phase.

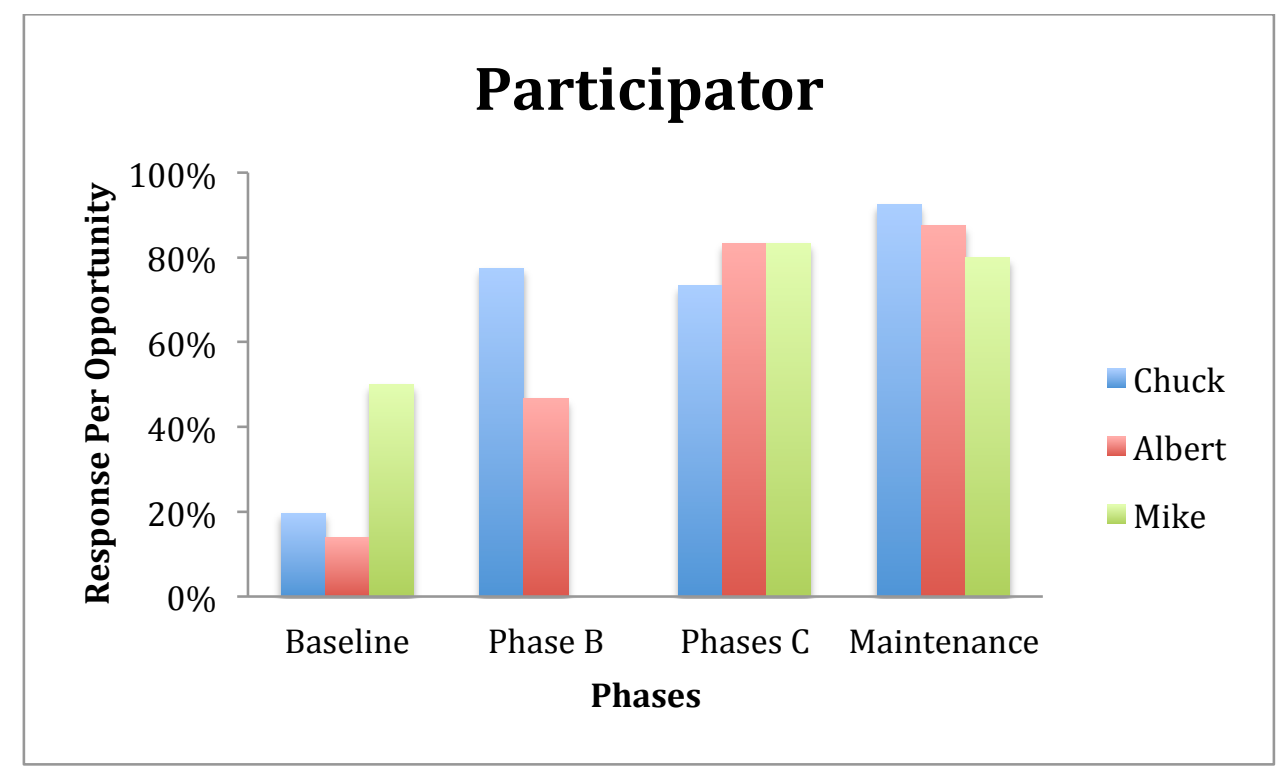

Figure 5. The Means for Child Participants' Participator Repertoire

\section{Child Participants' Performance in Problem Solver Repertoire}

Table 13 displays the mean with ranges in parentheses and levels italics for the percent of "problem solver" repertoire. Chuck and Albert both show overall increases from baseline to maintenance phases. Both increased the means from $2.5 \%, 92.50 \%$, and $96 \%$ respectively for baseline, MLT and maintenance phases. Albert increased the percent of problem solver repertoire from baseline (3.64\%) to MLT $(92.50 \%)$, and from MLT $(92.50 \%)$ to maintenance $(97.50 \%)$. Mike displayed an increase in problem solver repertoire from baseline (5.71\%) to MLT (92.50\%) but a decrease from MLT (92.50\%) to maintenance 
(85\%). According to the study design, when Model-Lead-Test training shows a greater positive impact than Oral Lecture Discussion in Talker and Participator phases, OLD will not be applied or measured during the problem solver phase. In other words, Chuck's father and Albert's mother both performed in a superior manner during MLT as compared to OLD during Talker and Participator after they received MLT training.

Table 13

Means, Ranges, and Levels of Children's Percent of Problem Solver Repertoire

\begin{tabular}{lcccc}
\hline Child & $\begin{array}{c}\text { Phase A } \\
\text { (Baseline) }\end{array}$ & $\begin{array}{c}\text { Phase B } \\
\text { (Training MLT) }\end{array}$ & $\begin{array}{c}\text { Phase C } \\
\text { (Training OLD) }\end{array}$ & $\begin{array}{c}\text { Phase D } \\
\text { (Maintenance) }\end{array}$ \\
\hline Chuck & $2.5 \%$ & $92.50 \%$ MLT & N/A & $96 \%$ \\
& $(0-10)$ & $(90-100)$ & & $(90-100)$ \\
Albert & $0 \%$ & $90 \%, 90 \%$ & & $100 \%$ \\
& $3.64 \%$ & $95 \% \mathrm{MLT}$ & $\mathrm{N} / \mathrm{A}$ & $97.50 \%$ \\
Mike & $(0-10)$ & $(90-100)$ & & $(90-100)$ \\
& $0 \%$ & $90 \%, 100 \%$ & & $90 \%, 100 \%$ \\
& $5.71 \%$ & $92.50 \%$ & N/A & $85 \%$ \\
& $(0-10)$ & $(80-100)$ & & $(90-100)$ \\
& $10 \%$ & $80 \%, 100 \%$ & & $40 \%, 100 \%$ \\
\hline
\end{tabular}

Figure 6 below is a bar graph illustrating the means of child participants' problem solver repertoires during the study in each phase when the parent participants were implementing fading techniques with them. In general, the problem solver repertoire increased across baseline, from MLT and maintenance phases for all three child participants, with the exception of Mike. There was a slight decrease $(92.50 \%$ to $85 \%)$ in the maintenance phase. 


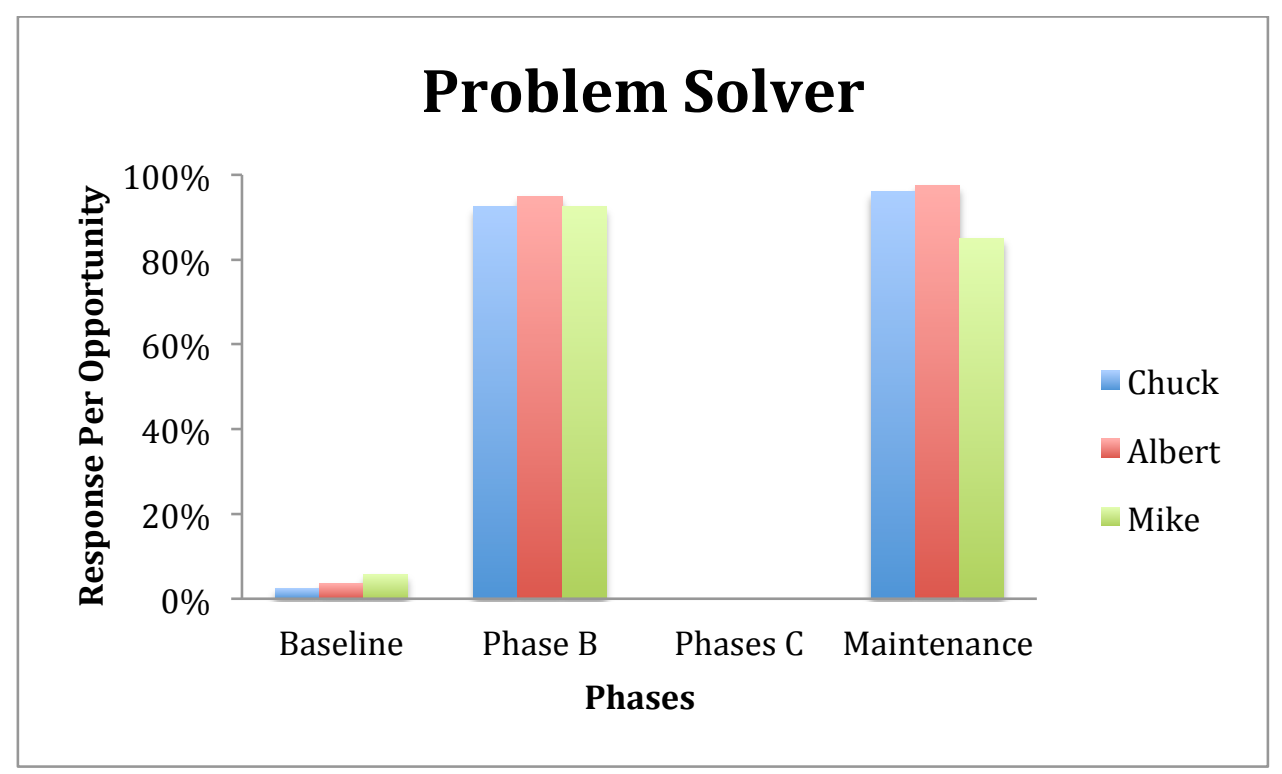

Figure 6. The Means of Child Participators' Problem Solver Repertoire

\section{Social Validity}

All 3 participants who completed the training also completed the Social Validity Questionnaire sheets. The Social Validity Questionnaire is a 5 point Likert scale that measures parental agreement with each particular question asked. The scale reflects the amount of agreement for each question in the following manner: $1=$ Strongly disagree; $2=$ Disagree; 3 = Neutral; $4=$ Agree; 5 = Strongly agree. The social validity questionnaire included 8 questions in both OLD and MLT sheets. Tables 16 and 17 show the results that each parent participant scored on the social validity sheets. Participant Mark and Brandy filled out the OLD sheet and rated all eight items as a five (Strongly agree), which indicated a strong agreement for each question. Participant Jenny did not participate in the OLD training due to the research protocol. All of the participants completed the Social Validity sheet for MLT training and rated all eight items as a five (Strongly agree). There remained one exception, Jenny, who selected three for "The interventions were easy to implement" which reflects "neutral" for that particular question. 
Table 14

Social Validity Questionnaire for Parents in OLD

\begin{tabular}{|l|c|c|c|}
\hline Question for Parent to Answer & Mark & Brandy & Jenny \\
\hline Appropriateness of Procedures & 5 & 5 & N/A \\
\hline $\begin{array}{l}\text { 1. The Parent Training handout was easy } \\
\text { to read and understand }\end{array}$ & 5 & 5 & N/A \\
\hline $\begin{array}{l}\text { 2. My trainer understood and } \\
\text { communicated procedures and techniques } \\
\text { effectively. }\end{array}$ & 5 & 5 & N/A \\
\hline $\begin{array}{l}\text { 3. The interventions were easy to } \\
\text { implement. }\end{array}$ & 5 & 5 & N/A \\
\hline Social Significance of Goals & 5 & 5 & N/A \\
\hline $\begin{array}{l}\text { 4. I would recommend a similar } \\
\text { intervention to other parents. }\end{array}$ & 5 & 5 & NA \\
\hline $\begin{array}{l}\text { 5. It is important to learn therapeutic } \\
\text { interventions to teach my child skills. }\end{array}$ & 5 & 5 & NA \\
\hline Social Importance of the Effects & & & \\
\hline $\begin{array}{l}\text { 6. The skills learned by my child are } \\
\text { beneficial to their development. }\end{array}$ & 5 & 5 & NA \\
\hline $\begin{array}{l}\text { 7. I will continue to use these } \\
\text { interventions with my child when a new } \\
\text { skill needs to be taught. }\end{array}$ & & & \\
\hline 8. My child learned the skills effectively. & 5 & 5 & \\
\hline
\end{tabular}


Table 15

Social Validity Questionnaire for Parent in MLT

\begin{tabular}{|l|c|c|c|}
\hline Question for Parent to Answer & Mark & Brandy & Jenny \\
\hline Appropriateness of Procedures & 5 & 5 & 5 \\
\hline $\begin{array}{l}\text { 1. The Model-Lead-Test procedure was } \\
\text { easy to read and understand }\end{array}$ & 5 & 5 & 5 \\
\hline $\begin{array}{l}\text { 2. My trainer understood and } \\
\text { communicated procedures and techniques } \\
\text { effectively. }\end{array}$ & 5 & 5 & 3 \\
\hline $\begin{array}{l}\text { 3. The interventions were easy to } \\
\text { implement. }\end{array}$ & 5 & 5 & 5 \\
\hline Social Significance of Goals & 5 & 5 & 5 \\
\hline $\begin{array}{l}\text { 4. I would recommend a similar } \\
\text { intervention to other parents. }\end{array}$ & 5 & 5 & 5 \\
\hline $\begin{array}{l}\text { 5. It is important to learn therapeutic } \\
\text { interventions to teach my child skills. }\end{array}$ & 5 & 5 & 5 \\
\hline Social Importance of the Effects & & & \\
\hline $\begin{array}{l}\text { 6. The skills learned by my child are } \\
\text { beneficial to their development. }\end{array}$ & 5 & 5 & 5 \\
\hline $\begin{array}{l}\text { 7. I will continue to use these } \\
\text { interventions with my child when a new } \\
\text { skill needs to be taught. }\end{array}$ & & & \\
\hline 8. My child learned the skills effectively. & & & \\
\hline
\end{tabular}




\section{Chapter Four}

\section{Discussion}

There is a substantial increase in the total number of children diagnosed with Autism Spectrum Disorder as reported by schools, clinics, and service agencies worldwide (Bax, 1994; Cox, Klein, \& Charman, 1999, Department of Developmental Service, 1999; Croen, Grether, Hoogstrate, \& Selvin, 2002). Rearing a child with Autism Spectrum Disorder is one of the most difficult, exhausting and heartbreaking experiences for any parent or family member (Heward, 2006). However, much of the stress inherent in the rearing process can be reduced by becoming proficient and more confident in their abilities in helping their learners with autism. Parent training definitely enhances parents' self-efficacy in both interacting with their children and helping them to improve skills in daily life.

The purpose of this study was to evaluate the effectiveness of the core content of Applied Behavior Analysis, or "...technology of behavior change" (Jenson, Sloane \& Young, 1988) for parents of learners with Autism Spectrum Disorder through "Model-Lead-Test" training via a multiple baseline across-behavior design. Several results found in this study are remarkable. The outcomes illustrate improvements in both parent and child participants. MLT in this study has proven to be effective in discrimination training, prompting and fading as also seen in the Peterson study (Peterson, McLaughlin, Weber, and Anderson, 2008). This supports the feasibility and preliminary efficacy of this six month parent training for applying reinforcement, prompting, and fading for learners with Autism Spectrum Disorder to improve the "talker, participator, and problem solver" repertoires. This study has also shown that Coaching used in the MLT protocol is helpful to the internal processes and external interactions of the parent and child. This finding corroborates the data found in the Greer study of the effects of coaching as well (Greer, 2002). The data collected from this study demonstrates and replicates the same findings for the importance of parent training as 
illustrated in the Competent Learner Model that has effectively served numerous children with autism (Tucci, Hursh \& Laitinen, 2004). The following discussion describes the outcomes for each research question. In addition, limitations in the study and future research directions are discussed.

\section{Is Parent Training Using a Model-Lead-Test Procedure Effective in Increasing Parents'} Appropriate Use of Reinforcement, Prompting, and Fading Techniques?

Figure 1 illustrates the first results of "reinforcement" training using baseline, OLD training, MLT and maintenance data. We find the data remains at near baseline levels when using OLD training techniques. A sharp increase in the data is seen when using MLT techniques and continues this steady level during maintenance. In the beginning the average shifts from 5\% to $9.25 \%$ after the parent received the OLD training. After receiving MLT training, Mark adapted the strategies from the researcher, and the means increased from $9.25 \%$ to $86.67 \%$. The second training for "prompting" techniques uses baseline, MLT, OLD and maintenance. The data reflects a low response level with a downward trend in the baseline. Before Mark had a training, he usually said to his learner "Say what I say," or "Look at me," "Say the whole thing," and/or, "You say it," if the learner didn’t respond as expected. He very often kept repeating the same sentence for a few times and usually didn't pause long enough time for the learner to process. Very often Mark spent more than 1 minute to encourage or prompt the learner to respond to each task during observations. Upon MLT intervention, we find the data sharply increases using proper prompting techniques, as the data continues the level using OLD (means $87.76 \%$, range $80-100 \%$ ) techniques and remains at high levels during maintenance. The third training involves fading techniques followed by MLT only. The researcher decided to use this design because observations of previous designs indicated that MLT alone had a high impact. The researcher also added an additional week of observation to the 4 regularly scheduled weekly observations during maintenance in 
reinforcement training session. During this session, the child participant's brother interrupted the normal training session activities by crying and making noise. Because of this, the father was not able to concentrate. A fifth session was then added to validate the results of previous sessions to prove validity. The 5 th week of observation was added to test and control for the decrease seen in the maintenance phase.

Figure 2 illustrates the results of training using baseline, MLT, OLD and maintenance for "reinforcement" techniques. Baseline reflects 0 response when the learner had correct behavior. Brandy only responded correctly $60 \%$ of the time in her first trial in applying reinforcement with specific praise because she was nervous in front of the video camera. Therefore, she gave her child participant tokens and said "good job", but didn't feel comfortable continuing to praise him more specifically. She practiced for a few days and responded correctly $100 \%$ of the time in her second trial when the researcher returned and maintained a high response rate (80\%-100\%) in the rest of MLT sessions and OLD and maintenance phases as well. Upon intervention, we find a sharply increased level with a correspondingly increasing trend. Similar results occurred with OLD training and continued with maintenance. In the second training using prompting techniques, baseline is a low response level using prompting, then an increasing level using OLD with a decreasing trend. Most of the time, Brandy would say, "try again", and/or "listen" if Albert didn't respond correctly. Then a further improvement in the data can be seen during MLT sessions with 0 trend for $100 \%$ response per opportunities. Maintenance displays a continued high response level. Based on the results of the previous two procedures, the researcher proceeded to extend baseline until results showed which training had the greater impact. Interestingly, baseline during fading procedures reflects a response level that is a marginal increase over 0 responses. This outcome was observed by the researcher as a result of previous OLD training ("least-tomost" prompting concept). The participant tried to use less prompting to the learners' 
behavior but was limited by lack of knowledge with fading concept. As soon as MLT was introduced, the researcher found the data increased significantly and held a steady trend across MLT and maintenance. Based on the conclusions derived from Mark and Brandy, the researcher has determined that the order in which conditions are presented is of paramount importance. MLT needs to be implemented before OLD is implemented in order to see improved results. The results also indicate that MLT alone can produce maximum effects without having OLD used.

Figure 3 illustrates the results of training using baseline, MLT and maintenance. In the design with Jenny, we base the selection of the type of coaching for her on the more effective type of coaching found with the other two participants. Thus the MLT technique along with maintenance was used exclusively. However, the session was shortened unexpectedly due to the participant's work schedule. Baseline presents at low level, "0-20\%", with a sharp increase in data across the whole study. This includes the implementation of reinforcement, prompting and fading techniques across MLT and maintenance (One exceptional data point is found in the fading maintenance phase due to the family returning from vacation when parent participant was tired and sick). Also, in the beginning of the study, Jenny was observed not smiling when she interacted with Mike. She usually didn't provide prompts at all if Mike responded incorrectly due to a history of Mike's maladaptive behavior. She would just move to the next sentence or question instead of asking Mike to answer. She also complained to the researcher about her frustration with managing her learner's nonparticipation behaviors. However, in the maintenance phase across reinforcement, prompting and fading sessions, Jenny appeared impressed by her son's abilities. She was observed excitedly saying "Wow, that's great Mike, you are helping me with the dishes", or "Wow, very nice Mike, good repeating". Thus, we find the data reflects a change in behavior for the child participant as well as the behavior of the parent. Thus, the training appears to 
inadvertently affect the parents behavior as a by-product of implementing ABA techniques with the children.

Do the Learner's Talker, Participator, and Problem Solver Repertoires Improve for Learners with Autism Spectrum Disorder as Their Parents' Appropriate Use of Reinforcement, Prompting, and Fading Techniques Increase?

Figure 4, bar graph, illustrates the mean data for child participants' "talker" repertoire when the parent participants were using reinforcement techniques. In the baseline, all three participants exhibited low motivation to repeat or answer their parents' questions and resulted in low or moderate scores in their correct response. The means outcomes were $36.67 \%, 60 \%$, and $67.50 \%$ for Chuck, Albert and Mike. During the baseline session, Albert complained that the task was boring and he appeared disengaged much of the time during observation. Afterward, Brandy started using specific statements of praise with the token system. Albert exhibited excitement and several times he praised himself, "I am doing so great", and said, "I can do it" and "I am so proud of myself" when he responded correctly. Overall, these techniques, OLD and MLT, appeared to be effective and increase "talker" repertoires, $80 \%$, $90 \%$, and $92.50 \%$ in maintenance phases for the three child participants.

Figure 5, bar graph, illustrates the mean data for child participants' "participator" repertoire when the parents are implementing prompting techniques. In the beginning of the study, three child participants had a lot of maladaptive behaviors, whining, sobbing, stomping feet, dropping on the floor, disassociating, and absconding from the designated area. The happened, especially, when they answered incorrectly and the parents kept using the ineffective prompts. Overall, these techniques appeared to be especially effective for Chuck and Albert. The researcher observed that the mean data for Chuck increased significantly from 19.69 to 92.5 percent. And the mean data for Albert increased from 14 to 87.5 percent. 
Figure 6, bar graph, illustrates the mean data for child participants' "problem solver" repertoire when parents are using fading technique. The data results show a remarkable increase due to fading training. Chuck increased from 2.5 to 96 percent, Albert increased from 3.64 to 97.5 percent, and Mike increased from 5.71 to 85 percent.

In conclusion, all three parent participants increased their implementation of reinforcement, prompting, and fading especially through MLT training. The child participants improved their talker, participator, and problem solver repertoires as well across parental reinforcement, prompting and fading training. The results of this study are not only appreciable during the training but may prove to be just as valuable during the practical application of these same techniques in a home setting. The improvement in the learner's repertoires may be seen as a direct result of the parent training. The rationale behind the multiple baseline design intervention is that change in the behavior is evident when and only when the researcher changes the conditions; thus eliminating the possibility that other variables are responsible for the behavior change. Therefore, the researcher observed the behavior was stable, then changed the intervention only at that time so that the researcher was sure that no other variables were responsible for the change. This can be asserted due to the consistent changes across skills, participants, and points in time.

\section{Limitations and Discussions for Future Research}

There are some limitations of this study that should be considered while interpreting the outcomes.

First, the child participants have maladaptive behaviors, which included aggression to others, self-injurious behaviors, screaming, whining, and so on. During the data collection period, parent participants would accommodate child participants' needs to reduce the maladaptive behaviors. For example, Mark offered his child participant, Chuck, some favorite food before sessions started because he was hungry. After Chuck was satiated, it 
impacted his motivation to participate. There were a few sessions Chuck left the designated areas and refused to interact with Mark. This condition also impacted the outcomes of child participant's performance.

Secondly, a portion of the data collection periods took place during summer vacation. The families sometimes canceled the prearranged visiting dates. Very often the child participants were observed not wanting to participate in the study after having long breaks. There were 3 times during the data collection in Chuck's sessions that had to be rescheduled due to his refusal to attend the sessions. Brandy also cancelled at least 7 appointments with the researcher.

Third, there were a very small number of participants in this study. All three parent participants had volunteered to be trained, but they were not chosen randomly. Therefore, they were highly motivated to learn ABA techniques to help their learners. And the sessions were conducted in prearranged environments rather than random situations thus the data reflects the higher motivation of parents willing to engage and help with their own children. Because these situations were arranged with willing and ready parents, the data reflects a high amount of motivation. The results of parent participants showed positive gains across all three of them. All of the questions in the social validity questionnaire for both OLD and MLT were marked strongly agree with one exception, Jenny, who scored 3 for "The interventions were easy to implement" which reflects "neutral" for that particular question.

Fourth, in the design with Jenny, we based the selection of the type of coaching for her on the more effective type of coaching found with the other two participants. In the beginning the researcher tried to rule-out that the results for the third participant were not due to a shorter baseline and treatment phases. Unexpectedly, Jenny informed the researcher of a change in scheduled sessions which resulted in fewer than the desired number of sessions. 
Thus, one possible limitation in this case presents itself in the form of too few a number of trials.

Fifth, Mark and Brandy both understood the concept of reinforcement but preferred less to use edible items. Even though Albert would be highly motivated by sweet snacks, Brandy usually chose chips or pretzels during the study. Therefore, Albert's performance was not consistent despite Brandy following protocol to provide tokens and praise Albert specifically. Mark preferred to use social reinforcement with Chuck, which included tickling, giving high fives, and hugging to Chuck. Even though Chuck improved his talker repertoire during reinforcement training, it was not consistent.

In future procedural designs, the researcher would optimally design the study to avoid "vacation times" that appear to confound the data. It would appear that participants' time away from training has a deleterious effect on proficient performance. Irregular scheduling during vacation time correlates to maladaptive escape behaviors. Which then causes the family to cancel further sessions and unexpectedly extends the study time. In the future, the researcher would ideally include more participants as well as varying the child participants' ages. Anecdotally, the researcher has discussed with current clients the possible benefits of this training for older learners. The participating parents, although benefitting from training for their younger children, express concern that older learners would not receive as great a benefit because they have already matured along with the current reinforcers, thus their potential to be reinforced by those same things is greatly diminished. Further, the researcher has observed that parental "acceptance" of appropriate reinforcement is sometimes an impediment to implementation of an effective intervention. Different parents hold differing belief systems about how best to motivate their children. For example, some parents do not want to use "edibles" as reinforcement. While others do not want to accept the notion that material items, outside the realm of intrinsic motivation, actually influences the behaviors of 
their child. However, when we recruit our participants we are unable to predict what their philosophy of correct child rearing techniques may be. In future studies we may want to consider using a parent survey to determine their attitudes about child rearing if we want to control for this variable.

In conclusion, previously, the researcher (Welterlin, 2009) has used modeling techniques, didactic techniques and hands on techniques separately with parents and their learners with varying degrees of success. Following this study, the researcher now concludes that the effectiveness of using the core content of Applied Behavior Analysis via ModelLead-Test technique is a superior method of training for parents and their children with autism. The parents in the study unanimously agreed, displaying a high degree of social validity, that the training methods were a success and that they would continue to use the ABA techniques to improve their children's repertoires. Improving the behavioral repertoires of children with Autism Spectrum Disorder is in fact the main emphasis for one pioneering researcher, Vicci Tucci, BCBA, and her approach using the Competent Learner Model. The CLM specifically trains parents to effectively use ABA techniques in bridging the gap between the theory and practice of teaching individuals with autism (Tucci, Hursh, \& Laitinen, 2004). One of the practical outcomes of this study has shown the importance of emphasizing MLT instead of only using verbal instructions, which do not have much impact. Thus, the focus of future trainings should highlight MLT as an indispensible component of parent training.

However, even a parent with the best of intentions may find it overwhelming to tackle the problem of providing appropriate assistance to a special needs child without help and support. As Jenny, one parent in the study quipped, "It is easy to learn and implement ABA techniques in a study, but in real life it is more difficult to be consistent, because the hardest part is being a parent of a child with autism". 


\section{References}

Adamson, L. B., Romaski, M. A., Bakeman, R., \& Sevcik, R. A. (2010). Augment language intervention and emergency of symbol-infused joint engagement. Journal of Speech, Language, and Hearing Research, 53, 1769-1773.

Akyeampong, K., Pryor, J., \& Ghartey, A. J. (2006). A vision of successful schooling: Ghanaian teachers' understanding of learning, teaching and assessment. Comparative Education, 42(2), 155-176.

Alberto, P. A., \& Troutman, A. C. (2013). Applied behavior analysis for teachers $\left(9^{\text {th }} \mathrm{ed}.\right)$. Boston, MA: Pearson.

Aldred, C., Green, J., \& Adams, C. (2004). A new social communication intervention for children with autism: Pilot randomized controlled treatment study suggesting effectiveness. Journal of Child Psychology and Psychiatry, 45(8), 1420-1420.

American Psychiatric Association. (2014). Diagnostic and statistical manual of mental disorders ( $5^{\text {th }}$ ed., text rev.). Washington, DC: American Psychiatric Press.

Aram, D., \& Levin, I. (2001). Mother-child joint writing in low SES: Sociocultural factors, maternal mediation, and emergent literacy. Cognitive Development, 16(3), 831-852.

Autism Speaks. (2012). Autism's costs to the nation reach $\$ 137$ billion a year. Retrieved January 31, 2013 from http://www.autismspeaks.org/science/science-news/autism'scosts-nation-reach-137-billion-year

Bandura, A. (1970). Social learning theory. Englewood Cliffs, NJ: Prentice-Hall.

Bax, M. (1994). Editorial: Autism. Developmental Medicine and Child Neurology, 36, 659660.

Billet, S. (1994). Situating learning in workplace: Having another look at apprenticeship. Industrial and Commercial Training, 26(11), 9-16. 
Binder, C., \& Watkins, C. L. (1990). Precision teaching and direct instruction: Measurably superior instructional technology in schools. Performance Improvement Quarterly, 3(4), 74-96.

Blamey, K. L., Meyer, C. K., \& Walpole, S. (2008). Middle and high school literacy coaches: A national survey. Journal of Adolescent \& Adult Literacy, 52(4), 310-323.

Boyd, B. A. (2002). Examining the relationship between stress and lack of social support in mothers of children with autism. Focus on Autism and Other Developmental Disabilities, 17, 208-215.

Bradley, A. (2007). Federal study documents rates of autism disorder in 14 states. Education Week, 26(23), 6.

Bristol, M. M., \& Schopler, E. (1984). A development perspective on stress and coping in families of autistic children. In J. Blancher (Ed.), Severely handicapped children and their families (pp. 91-141). New York: Academic Press.

Britton, L. R., \& Anderson, K. A. (2010). Peer coaching and pre-service teachers: Examining an underutilized concept. Teaching and Teacher Education, 26(2), 306-314.

Bryson, S. E. (1996). Epidemiology of autism. Journal of Autism and Developmental Disorders, 26, 165-167.

Cazden, C. (1983). Adult assistance to language development: Scaffolds, models, and direct instruction. In R. Parker \& F. Davis (Eds.), Developing literacy: Young children's use of language (pp. 3-18). Newark, DE: International Reading Association.

Centers for Disease Control and Prevention. (2014, March 24). Autism Spectrum Disorder (ASD): Data \& statistics. Retrieved from http://www.cdc.gov/ncbddd/autism/data.html 
Cohen, N. H. (1995). Mentoring adult learners: A guide for educators and trainers.

Professional practices in adult education and human resource development series. Melbourne, FL: Krieger Publishing Co.

Cooper, J.O., Heron, T.E., \& Heward, W.L. (2007). Applied behavior analysis (2nd ed.). Upper Saddle River, NJ: Pearson.

Coulter, G. A., \& Grossen, B. (1997). The effectiveness of in-class instructive feedback versus after-class instructive feedback for teachers learning Direct Instruction teaching behaviors. Effective School Practices, 16(4), 21-34.

Cox, A., Klein, K., \& Clarman, T. (1999). Autism spectrum disorder at 20 and 42 months of age: Stability of clinical and ADI-R diagnosis. Journal of Child Psychology and Psychiatry, 40, 719-732.

Croen, L. A., Grether, J. K., Hoogstrate, J., \& Selvin, S. (2002). The changing prevalence of autism in California. Journal of Autism and Development Disorders, 32(3), 207-215.

Davis, N. O., \& Carter, A. S. (2008). Parenting stress in mother and father of toddlers with autism spectrum disorders: Associations with child characteristics. Journal of Autism Development Disorder, 38, 1278-1291.

Dennen, P. V. (2004). Cognitive apprenticeship in educational practice: Research on scaffolding, modeling, mentoring, and coaching as instructional strategies. In D. H. Jonassen (Ed.), Handbook of Research on Educational Communications and technology. Mahwah, NJ: Lawrence Erlbaum Associates.

Department of Developmental Services. (1999). Changes in the population of persons with autism and pervasive development disorders in California's developmental services system: 1978-1998. A report to the legislature. Sacramento, CA: Author. 
Desforges, C., \& Abouchaar, A. (2003). The impact of parental involvement, parental support and family education on pupil achievement and adjustment: A literature review. London, England: Department for Education and Skills.

Diouf, W., Sheckley, B., \& Kehrhahn, M. (2000). Influences on the learning of adults in Senegalese villages: Implications for practice and theory. Adult Education Quarterly, $51(1), 32-44$.

Dougherty, C., \& Tan, J. (1997). Financing training: Issues and options. International Journal of Manpower, 18(1), 29-62.

Dunn, M. E., Burbine, T., Bowers, C. A., \& Tantleff-Dunn, S. (2001). Moderators of stress in parents of children with autism. Community Mental Health Journal, 37, 39-52.

Dyson, L. L. (1993). Response to the presence of a child with disabilities: Parental stress and family functioning over the time. American Journal on Mental Retardation, 98, $207-$ 218.

Elder, J. H. (2002). Current treatments in autism: Examining scientific evidence and clinical implications. Journal of Neuroscience Nursing, 34(2), 67-73.

Fixsen, D. L., Naoom, S. F., Blase, K. A., Friedman, R. M., \& Wallace, F. (2005). Implementation research: A synthesis of the literature. Tampa, FL: University of South Florida, Louis de la Parte Florida Mental Health Institute, The National Implementation Research Network.

Forehand, R., \& Peed, S. (1979). Training parents to modify noncompliant behavior of their children. In A. J. Finch, Jr. \& P. C. Kendall (Eds.), Treatment and research in child psychopathology (pp. 159-184). New York, NY: Spectrum.

Formbonne, E. (2005). A changing epidemiology of autism. Journal of Applied Research in Intellectual Disabilities, 18(4), 281-294. 
Frazier Varner, D. (2010). Effect of instructional design on academic success of adult basic education learners: Individualized versus group design (Unpublished doctoral dissertation). Walden University, Minneapolis, MN.

Gibaud-Wallston, J., \& Wandersman, L. P. (1978). Development and utility of the Parenting Sense of Competence Scale. Paper presented at the annual meeting of the American Psychological Association, Toronto.

Gillberg C., \& Billstedt E. (2000). Autism and Asperger syndrome: Coexistence with other clinical disorders. Acta Psychiatr Scand, 102, 321-330.

Glover, P., McLaughlin, T. F., Derby, K. M., \& Gower, J. (2010). Using a direct instruction flashcard system employing a back three contingency for errors with two students with learning disabilities. Electronic Journal of Research in Educational Psychology, $8,457-482$.

Gray, D. E. (2002). Then years on: A longitudinal study of families of children with autism. Journal of Intellectual and Developmental Disability, 27, 215-222.

Green, C., McLaughlin, T. F., Derby, K. M., \& Lee, K. (2010). Using reading racetracks and flashcards to teach sight words to students with disabilities: Effects for acquisition and response maintenance. Journal of Educational Research: JER, 13(2), 84-98.

Greer, R. D. (2002). Designing teaching strategies: An applied behavior analysis systems approach. San Diego, CA: Academic Press.

Grindle, C. F., Hastings, R. P., Saville, M., Hughes, J. C., Huxley, K., Kovshoff, H., . . . Remington, B. (2012). Outcomes of a behavioral education model for children with autism in a mainstream school setting. Behavior Modification, 36(3), 298-319.

Guiney, E. (2001). Coaching isn't just for athletes: the role of teacher leaders. Phi Delta Kappan, 82, 740-744. 
Gurney, J. G., McPheeters, M. L., \& Davis, M. M. (2006). Parental report of health conditions and health care use among children with and without autism. Archives of Pediatrics Adolescent Medicine, 160(8), 825-830.

Hastings, R. P., Kovshoff, H., Brown, T., Ward, N. J., Espinosa, F. D., \& Remington, B. (2005). Coping strategies in mothers and fathers of preschool and school-aged children with autism. Autism, 9, 377-391.

Heward, W. L. (1994). Three "low tech" strategies for increasing the frequency of active student responding during group instruction. In R. Gardner III, P. M. Sainato, J. O. Cooper, W. L. Heward, J. Eshelman, \& T. A. Grossi (Eds.), Behavior analysis in education: Focus on measurably superior instruction (pp. 283-320). Pacific Grove, CA: Brooks/Cole.

Heward, W. L. (2006). Exceptional children: An introduction to special education ( $8^{\text {th }}$ ed.). Upper Saddle River, NJ: Merrill/Prentice Hall.

Holroya, J., \& McArthur, D. (1976). Mental retardation and stress on the parents: A contrast between Down's syndrome and childhood autism. American Journal of Mental deficiency, 80, 431-436.

Horton, L. (1984). The father's role in behavioral parent training: A review. Journal of Behavior Therapy and Experimental Psychiatry, 9, 1-5.

Idol, L. (1987). Group story mapping: A comprehension strategy for both skilled and unskilled readers. Journal of Learning Disability, 20(4), 196-205.

Ingersoll, B., \& Dvortcsak, A. (2006). Including parent training in the early childhood special education curriculum for children with autism spectrum disorders. Journal of Positive Behavior Interventions, 8, 79-87. 
Iwata, B. A., Pace, G. M., Dorsey, M. F., Zarcone, J. R., Vollmer, T. R., Smith, .. . Willis, K. D. (1994). The function of self-injurious behavior: An experimental-epidemiological analysis. Journal of Applied Behavior Analysis, 27, 215-240.

Jenson, W. R., Slonae, H. N., \& Young, K. R. (1988). A structured teaching approach. Applied Behavior Analysis in Education. Englewood Cliffs, NJ: Prentice-Hall.

Johnston, C., \& Mash, E. J. (1989). A measure of parenting satisfaction and efficacy. Journal of Clinical Child Psychology, 18, 167-175.

Jones, L. (2009). A parent training program combining discrete trial training and incidental teaching in the home environment (Unpublished master's thesis). University of South Florida, Tampa, FL.

Kaiser, A., Hester P., Albert, C., \& Whiteman, B. (1995). Preparing parent trainers: An experimental analysis of effects on trainers, parents, and children. Topics in Early Childhood Special Education, 15, 385-414.

Kaufman, L., McLaughlin, T. F., Derby, K. M., \& Waco, T. (2011). Employing reading racetracks and DI flashcards with and without cover, copy, and compare and rewards to teach of sight words to three students with learning disabilities in reading. Educational Research Quarterly, 34, 24-44.

Kazdin, A. E. (2011). Single-case research designs: Methods for clinical and applied settings. New York, NY: Oxford University Press.

Keller, B. (2007). Coaching teachers to help students learn. Educational Week, 27(15), 22-24.

Kennedy, C. H. (2005). Single case designs for educational research. Boston, MA: Allyn \& Bacon.

Koegel, L. K., Carter, C. M., \& Koegel, R. L. (2003). Teaching children with autism selfinitiations as a pivotal response. Topics in Language Disorders, 23(4), 134-145. 
Koegel, L. K., Koegel, R. L., Harrower, J. K., \& Carter, C. M. (1999). Pivotal response intervention I: Overview of approach. The Journal of the Association for Persons with Severe Handicaps, 24(3), 174-186.

Koegel, R., Bimbela, A., \& Schreibman, L. (1996). Collateral effects of parent training on family interactions. Journal of Autism and Developmental Disorders, 26, 347- 359.

Koegel, R. L., Brookman, L., \& Koegel, L.K. (2003). Autism: Pivotal response intervention and parent empowerment. Trends in Evidence-based Neuropsychiatry, 61-69.

Koegel, R. L., Glahn, T. J., \& Nieminen, G. W. (1978). Generalization of parent training results. Journal of Applied Behavior Analysis, 11, 95-109.

Koegel, R. L., \& Koegel, L. K. (1987). Generalization issues in the treatment of autism. Seminars in Speech and Language, 8, 241-256.

Koegel, R. L., O’Dell, M., \& Koegel, L.K. (1987). A natural language paradigm for nonverbal autistic children. Journal of Autism and Developmental Disorders, 17(2), $187-200$.

Koegel, R. L., Schreibman, L., Britten, K. R., Burke, J. C., \& O’Nell, R. E. (1982). A comparison of parent to direct clinic treatment. In R. L. Koegel, A. Rincover, \& A. L. Egel (Eds.), Educating and understanding autistic children (pp.260-279). San Diego, CA: College Hill Press.

Konstantareas, M. M., \& Homatidis, S. (1989). Assessing child symptom severity and stress in parents of autistic children. Journal of Child Psychology and Psychiatry, 30, 459470.

Kupzyk, S., McCurdy, M., Hofstadter, K. L., \& Berger, L. (2011). Recoded readings: A taped parent-tutoring intervention. Journal of Behavioral Education, 20(2), 87-102.

Lajoie, S. (2005). Extending the scaffolding metaphor. Instruction Science, 33(5), 541-557. 
Lerman, D. C., Swiezy, N., Perkins-Parks, S., \& Roane, H. S. (2000). Skill acquisition in parents of children with developmental disabilities: Interaction between skill type and instructional format. Research in Developmental Disabilities, 21, 183-196.

Levy, S. E., Giarelli, E., lee, L., Schieve, L. A., Kirby, R. S., Cummiff, C., Nicholas, J., . . Rice, C. E. (2010). Autism spectrum disorder and co-occurring developmental, psychiatric, and medical conditions among children in multiple populations of the United States. Journal of Development Behavioral Pediatrics, 31(4), 267-275.

Lindsey, J. (2009). A parent training program combining discrete trial training and incidental teaching in the home environment. Graduate School Theses and Dissertations. Retrieved September 10, 2012 from http://scholarcommons.usf.edu/etd/2033

Luong, N. (2008). Family support for at-risk second graders to improve reading fluency (Submitted in partial fulfillment of the requirements for degree). Dominican University of California, San Rafael, CA.

Lovaas, O. I. (1987). Behavioral treatment and normal educational and intellectual functioning in young autistic children. Journal of Consulting and Clinical Psychology, $55,3-9$.

Lovaas, O. L. Koegel, R. L., Simmons, J. O., \& Long, J. S. (1973). Some generalization and follow-up measure on autistic children in behavior therapy. Journal of Applied Behavior Analysis, 6, 131-165.

Machand-Martella, N., Slocum, T. A., \& Martella, R. (Eds.). (2004). Introduction to direct instruction. Boston, MA: Allyn-Bacon.

Malfa, G. L., Bertelli, L. M., Salvini, R., \& Placidi, G. F. (2004). Autism and intellectual disability: A study of prevalence on a sample of the Italian population. Journal of Intellectual Disability Research, 48(3), 262-267. 
Malott, R. W., \& Suarez, E. T. (2004). Principles of behavior. Upper Saddle River, NJ: Pearson.

Mancil, G. R., Boyd, B.A., \& Bedersem, P. (2009). Parental stress and autism: Are there useful coping strategies? Education and Training in Developmental Disabilities, 44(4), 523-537.

Marcus, L., Lansing, M., Andrews, C., \& Schopler, E. (1978). Improvement of teaching effectiveness in parents of autistic children. Journal of the American Academy of Child Psychiatry, 17, 625-639.

McConachie, H., \& Diggle, T. (2007). Parent implemented early intervention for young children with autism spectrum disorder: a systematic review. Journal of Evaluation in Clinical Practice, 13, 120-129.

McCuller, G. (2002). Preparing personnel to work with children with serious emotional disturbance and autism (Final Report, Grant \#H32511990124). Retrieved from Education Resources Information Center (ERIC).

McGrath, P. (2006). Psychosocial issues in childhood autism rehabilitation: A review. International Journal of Psychosocial Rehabilitation, 11(1), 29-36.

Miller, L. K. (2006). Principles of everyday behavior analysis $\left(4^{\text {th }}\right.$ ed.). Belmont, CA: Wadsworth.

Miller, L. K., \& Weaver, F. H. (1975). The use of “concept programming” to teach behavioral concepts to university students. In J. Johnson (Ed.), Behavior Research and Technology in higher education. Springfield, IL: Charles C. Thomas.

Miltenberger, R. G. (2004). Behavior modification: Principles and procedures $\left(3^{\text {rd }}\right.$ ed.). Belmont, CA: Wadsworth /Thomson Learning.

Minnes, P., \& Woodford, L. (2005). Well-being in aging parents caring for an adult with a developmental disability. Journal on Developmental Disabilities, 11(1), 47-66. 
Mohler, G. M., Yun, K. A., Carter, A., \& Kaska, D. (2009). The effect of curriculum, coaching, and professional development on prekindergarten children's literacy achievement. Journal of Early Childhood Teacher Education, 30(1), 49-68.

Moran, D.J., \& Malott, R.W. (2004). Evidence-based educational methods: Advances from the behavioral sciences. New York, NY: Academic Press.

Mudford, O.C., Martin, N. T., Eikeseth, S., \& Bibby, P. (2001). Parent-managed behavioral treatment for preschool children with autism: Some characteristics of UK programs. Research in Developmental Disabilities, 22(3), 173-182.

Mundy, P., Sigman, M., \& Kasari, C. (1994). Joint attention, developmental level, and symptom presentation in young children with autism. Development and Psychopathology, 6, 389-401.

National Research Council. (2001). Educating children with autism. Committee on educational interventions for children with autism. C. Lord \& J. P. McGee (Eds.). Washington, DC: National Academy Press.

Neef, N. A., Marckel, J., Ferreri, S., Jung, S., Nist, L., \& Armstrong, N. (2004). Effect of modeling versus instructions on sensitivity to reinforcement schedules. Journal of Applied Behavior Analysis, 37(3), 267-281.

Neumann, M. N., \& Neumann, D. L. (2010). Parental strategies to scaffold emergent writing skills in the pre-school child within the home environment. Early Years, 30(1), 79-94.

Peterson, L., McLaughlin, T. F., Weber, K. P., \& Anderson, H. (2008). The effects of a model, lead, and test technique paired with visual prompts with a fading procedure to teach "where" to a 13-year-old echolalic boy with autism. Journal of Developmental and Physical Disabilities, 20, 31-39. 
Pratt, M. W., Kerig, P., Cowan, P. A., \& Cowan, C. P. (1988). Mothers and fathers teaching 3-year-olds: Authoritative parenting and adult scaffolding of young children's learning. Developmental Psychology, 24, 832-839.

Pratt, M. W., Green, D., MacVicar, J., \& Bountrogianni, M. (1992). The mathematical parent: Parental scaffolding, parent style, and learning outcomes in long-division mathematics homework. Journal of Applied Developmental Psychology, 13(1), 17-34.

Quill, K. A. (1995). Teaching children with autism: Strategies to enhance communication and socialization. New York, NY: Delmar Publishing.

Raj, A., \& Salagame, K. K. (2010). Effect of sensitized coaching on self-efficacy of parents of children with autism. Journal on Developmental Disabilities, 16(2), 44-51.

Reinke, W. M., Lewis-Palmer, T., \& Martin, E. (2007). The effect of visual performance feedback on teacher use of behavior-specific praise. Behavior Modification, 31(3), 247-263.

Roberts, M., \& Kaiser, A. (2011). The effectiveness of parent-implemented language intervention: A meta-analysis. American Journal of Speech-Language Pathology, 20, 180-199.

Rogers, S. J. (1998). Empirically supported comprehensive treatments for young children with autism. Journal of Clinical Child Psychology, 27(2), 168-79.

Ruwe, K., McLaughlin, T. F., Derby, K. M., \& Johnson, K. (2011). The multiple effects of direct instruction flashcards on sight word acquisition, passage reading, and errors for three middle school students with intellectual disabilities. Journal of Developmental and Physical Disabilities, 23, 241-255.

Sanders, J. L., \& Morgan, S. B. (1997). Family stress and adjustment as perceived by parents of children with autism or Down syndrome: For intervention. Child and Family Behavior Therapy, 19, 15-32. 
Sheinkopf, S., \& Siegel, B. (1998). Home-based behavioral treatment of young children with autism. Journal of Autism and Developmental Disorders, 28(1), 15-23.

Shidler, Linda. (2009). The impact of time spent coaching for teacher efficacy on student achievement. Early Childhood Education Journal, 36(5), 453-460.

Shook, G. L., \& Neisworth, J. T. (2005). Ensuring appropriate qualifications for applied behavior analyst professionals: The behavior analyst certification board. Exceptionality 13(1), 3-10.

Shouse, H., Weber, K. P., McLaughlin, T. F., \& Riley, S. (2012). The effects of model, lead, and test and a reward to teach a preschool student with a disability to identify colors. Academic Research International, 2(1), 477-483.

Solish A., \& Perry A. (2008). Parents' involvement in their children's behavioral intervention programs: Parent and therapist perspectives. Research in Autism Spectrum Disorders. 2(4), 728-738.

Stahmer, A. C., \& Gist, K. (2001). Enhancing parent training through additional support services. Journal of Positive Behavioral Support, 3, 75-82.

Tucci, V. (2006). Introductory CLM unit-Competent Learner Model. Watsonville, CA: Tucci Learning Solutions, Inc.

Tucci, V., Hursh, D. E., \& Laitinen, R. E. (2004). The Competent Learner Model (CLM): A merging of applied behavior analysis, direct instruction and precision teaching. In D.J. Moran \& R.W. Mallot (Eds.), Evidence-Based Educational Methods (pp.109-123). San Diego, CA: Elsevier Academic.

Ward, A. R., \& Zembal-Saul, C. (2007). Scientist-science teacher educator partnerships for developing and teaching science courses for preservice elementary teachers. Pennsylvania Teacher Educator, 6, 1-9. 
Watkins, C. L. (1988). Project Follow Through: A story of the identification and neglect of effective instruction. Youth Policy, 10(7), 7-11.

Welterlin, A. (2009). The home TEACCHING program: A study of the efficacy of a parent training early intervention model (Unpublished doctoral dissertation). Rutgers, State University of New Jersey, Newark, NJ.

Werts, M. G., Caldwell, N. K., \& Wolery, M. (1996). Peer modeling of response chains: Observational learning by students with disabilities. Journal of Applied Behavior Analysis, 29(1), 53-66.

Whitaker, S. (2002). Maintaining reductions in challenging behaviors: A review of the literature. The British Journal of Development Disabilities, 48(1), 15-25.

Wilder, D. A., Atwell, J., \& Wine, B. (2006). The effects of varying levels of treatment integrity on child compliance during treatment with a three-step prompting procedure. Journal of Applied Behavior Analysis, 39(3), 369-373.

Wolf, L. C., Noh, S., Fisman, S. N., \& Speechley, M. (1989). Brief report: Psychological effects of parenting stress on parents of autistic children. Journal of Autism and Developmental Disorders, 19(1), 157-166. 
Appendix A

Social Validity Questionnaire for Parents in OLD

Name:

Date:

\begin{tabular}{|c|c|c|c|c|c|}
\hline Question for Parent to Answer & 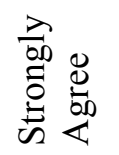 & 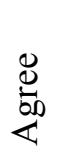 & 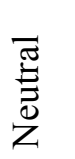 & 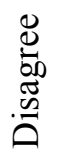 & 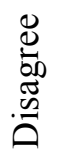 \\
\hline Appropriateness of Procedures & 5 & 4 & 3 & 2 & 1 \\
\hline $\begin{array}{l}\text { 1. The Parent Training handout was } \\
\text { easy to read and understand }\end{array}$ & & & & & \\
\hline $\begin{array}{l}\text { 2. My trainer understood and } \\
\text { communicated procedures and } \\
\text { techniques effectively. }\end{array}$ & & & & & \\
\hline $\begin{array}{l}\text { 3. The interventions were easy to } \\
\text { implement. }\end{array}$ & & & & & \\
\hline Social Significance of Goals & 5 & 4 & 3 & 2 & 1 \\
\hline $\begin{array}{l}\text { 4. I would recommend a similar } \\
\text { intervention to other parents. }\end{array}$ & & & & & \\
\hline $\begin{array}{l}\text { 5. It is important to learn therapeutic } \\
\text { interventions to teach my child skills. }\end{array}$ & & & & & \\
\hline Social Importance of the Effects & 5 & 4 & 3 & 2 & 1 \\
\hline $\begin{array}{l}\text { 6. The skills learned by my child are } \\
\text { beneficial to their development. }\end{array}$ & & & & & \\
\hline $\begin{array}{l}\text { 7. I will continue to use these } \\
\text { interventions with my child when a new } \\
\text { skill needs to be taught. }\end{array}$ & & & & & \\
\hline $\begin{array}{l}\text { 8. My child learned the skills } \\
\text { effectively. }\end{array}$ & & & & & \\
\hline
\end{tabular}


Appendix B

Recruitment Letter

Dear Parent,

This letter is a request for you to take part in a research project, which will provide free parent training for supporting the development of some of your child's Competent Learner Repertoires. The parent training will include a Board Certified Behavior Analyst coming to your home to work with you on learning some Applied Behavior Analysis techniques (Reinforcement, Prompting, and Fading) for establishing and strengthening your learner's desirable behaviors and as well weakening your learner's undesirable behaviors.

Your involvement in this project will be kept as confidential as legally possible. You must be 18 years of age or older and your child has to be between $4-12$ years old to participate. Your participation will involve sessions of 15 minutes to one hour one to five times per week on a schedule that works for you. This will last approximately 3-6 months. If you are interested in participating this study, please respond to the questions below and give this letter to the coordinator in your learner's case. We will give your contact information to the researcher, Liyu Chen. Giving this letter to the coordinator does not obligate you to participate in the study. It is only to allow us to give your contact information to the researcher. She will then contact you with more details about the study and to make sure you are eligible.

1. Do I have your permission to give Liyu Chen your contact information? $\square$ Yes $\square$ No

2. When is the best time for her to contact you?

\section{$\square$ Morning $\square$ Afternoon $\square$ Evening}

3. Your name: Date: Phone:

I hope that you will participate in this research project, as it could be beneficial in understanding the impact of coaching techniques on your learning to work effectively with your child. Thank you very much for your time. Should you have any questions about this letter or the research project, please feel free to contact the primary researcher, Liyu Chen at 321-795-2889 or by e-mail at lchen@tuccionline.com

Thank you for your time and help with this project.

Sincerely,

Liyu Chen, MS, BCBA

Tucci Learning Solutions, Inc. 


\author{
Appendix C \\ Parent Consent Form
}

\title{
W. WestVirginiaUniversity。
}

\author{
Human Research Protocol \\ Only Minimal Risk Consent \\ Parental or Guardian \\ (Without HIPAA)
}

\author{
Only Minimal Risk \\ Parental or Guardian Consent (Without HIPAA) \\ Principal Investigator \\ Dan Hursh \\ Department \\ Learning Sciences and Human Development \\ Protocol Number \\ 1311128662 \\ Study Title \\ The impact of Model-Lead-Test Coaching on Parents' use of Reinforcement, \\ Prompting, and Fading with Their Children with Autism Spectrum Disorder \\ Co-Investigator(s) Liyu Chen \\ Sponsor (if any) N/A
}

\section{Contact Persons}

In the event your child experiences any side effects or injury related to this research, you should contact Ruth Broomall at (831) 786-0600. After hours contact: Ruth Broomall at (408) 671-0035. If you have any questions, concerns, or complaints about this research, you can contact Dr. Dan Hursh at (304) 293-2076 or Liyu Chen 321795-2889.

For information regarding your child's rights as a research participant, to discuss problems, concerns, or suggestions related to the research, to obtain information or offer input about the research, contact the Office of Research Integrity and Compliance (304) 293-7073.

In addition if you would like to discuss problems, concerns, have suggestions related to research, or would like to offer input about the research, contact the Office of Research Integrity and Compliance at 304-293-7073.

Introduction

You, _ and your child, have been asked to participate in this research study, which has been explained to you and your child by Liyu Chen, MS. Dan Hursh, PhD and Liyu Chen, MS in the Department of Learning Sciences and Human Development at West Virginia University are conducting this study. This research is being conducted to fulfill the requirements for a doctoral dissertation for the Educational Psychology Program in the Department of Learning Sciences and Human Development at West Virginia University, under the supervision of Dan Hursh, PhD.

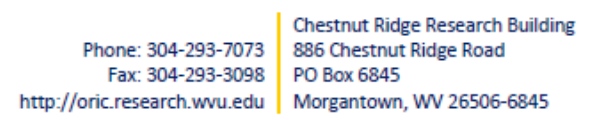

Page | 1

Subject's Initials

Date 


\section{W. WestVirginiaUniversity.}

Human Research Protocol
Only Minimal Risk Consent
Parental or Guardian

(Without HIPAA)

\section{Purpose(s) of the Study}

The purpose of this study is to learn more about evaluating the impact of Model-Lead-Test coaching on parents' used of reinforcement, prompting, and fading with their children with Autism Spectrum Disorder (ASD). Also another purpose is to assess whether there are improvements in the children's talker, participator, and problem solver repertoires associated with their parents' use of these behavior change processes. WVU expects to enroll approximately three to five parent-child pairs; a total of at least three parent-child pairs at all sites are expected to participate in this study.

\section{Description of Procedures}

This study will be done at your home. You will participate in a series of training sessions conducted by Liyu Chen. She will use Oral Lecture Discussion and Model-Lead-Test techniques to guide your use of reinforcement, prompting, and fading so as to develop your child's talker, participator, and problem solver repertoires. The sessions will last between 15 minutes and one hour one to five days each week for three to six months.

\section{Risks and Discomforts}

There are no known risks related to this study aside from any discomfort you may feel due to being observed or asked to answer questions about your interactions with your child and your participation in this research.

\section{Alternatives}

You and your child do not have to participate in this study.

\section{Benefits}

The benefits of this training are that you will learn how to use reinforcement, prompting, and fading to help your child develop his/her talker, participator, and problem solver repertoires.

\section{Financial Considerations}

No payments will be made for participating in the study.

\section{Confidentiality}

Any information about you or your child that is obtained as a result of their participation in this research will be kept as confidential as legally possible.

Your and your child's research records and test results, just like hospital records, may be subpoenaed by court order or may be inspected by the study sponsor or federal regulatory authorities without your additional consent.

Audiotapes or videotapes will be kept locked up and will be destroyed as soon as possible after the research is finished.

In any publications that result from this research, neither your or your child's name nor any information from which you or your child might be identified will be published without your consent.

\begin{tabular}{r|ll} 
& Chestnut Ridge Research Building & P a g e | 2 \\
Phone: $304-293-7073$ & 886 Chestnut Ridge Road & Subject's Initials__ \\
Fax: 304-293-3098 & PO Box 6845 & Date__
\end{tabular}


We- West VirginiaUniversity.
Human Research Protocol Only Minimal Risk Consent

Parental or Guardian (Without HIPAA)

\section{Voluntary Participation}

Refusal to participate or withdrawal will not affect your child's future care and will involve no penalty to you.

\section{Signatures}

Signature of Person Conducting Assent Discussion

$\begin{array}{lll}\text { Printed Name } & \text { Date } & \text { Time }\end{array}$

Statement of Parent or Guardian

My child appears to understand the research to the best of his or her ability and had agreed to participate.

Signature of Parent or Guardian

$\begin{array}{lll}\text { Printed Name } & \text { Date }\end{array}$

Upon signing this form, you will receive a copy. I willing consent to allow my child to participate in this research.

Signature of Parent or Guardian

$\begin{array}{lll}\text { Printed Name } & \text { Date }\end{array}$

The parent or guardian has had the opportunity to have questions addressed. The parent or guardian willingly agrees to allow his or her child to be in the study.

Signature of Investigator or Co-Investigator

\begin{tabular}{|c|c|c|c|c|}
\hline \multicolumn{2}{|l|}{ Printed Name } & Date & Time & \\
\hline & Chestnut Ridge Research Building & & & $\mathrm{Page} \mid 3$ \\
\hline Fax: 304-293-3098 & PO Box 6845 & & Subject's Initials & \\
\hline http://oric.research.wvu.edu & Morgantown, WV 26506-6845 & & Date & \\
\hline
\end{tabular}


Appendix D

Participation Assent Form

\section{W. WestVirginiaUniversity.}

Human Research Protocol

Assent Form

\section{Assent Form}

Principal Investigator

Dan Hursh

Department

Learning Sciences and Human Development

Protocol Number

1311128662

Study Title

The Impact of Model-Lead-Test Coaching on Parents' use of Reinforcement, Prompting, and Fading with Their Children with Autism Spectrum Disorder

Co-Investigator(s)

Liyu Chen

Sponsor (if any)

N/A

\section{Contact Persons}

If you are hurt from being in this research, you should contact Ruth Broomall at (831) 786-0600. After hours contact: Ruth Broomall at (408) 671-0035. If you have any questions, concerns, or complaints about this research, you can contact Dr. Dan Hursh at (304) 293-2076 or Liyu Chen 321-795-2889.

For information regarding your rights as a person in research or to talk about the research, call the Office of Research Integrity \& Compliance at (304) 293-7073.

\section{Introduction}

You, , have been asked to participate in this research study, which has been explained to you by Liyu Chen.

\section{Purpose(s) of the Study}

The primary purpose of this study is to evaluate the impact of Model-Lead-Test coaching on your parent's use of reinforcement, prompting, and fading with you. The secondary purpose is to assess whether there are improvements in your talker, participator, and problem solver repertoires associated with your parent's use of these behavior change processes.

\section{Description of Procedures}

This study will be done at your home. Your parents will participate in a series of training sessions conducted by Liyu Chen. She will use Oral Lecture Discussion and Model-Lead-Test techniques to guide your parent's use of reinforcement, prompting, and fading so as to develop your talker, participator, and problem solver repertoires. The sessions will last between 15 minutes and one hour one to five days each week for three to six months.
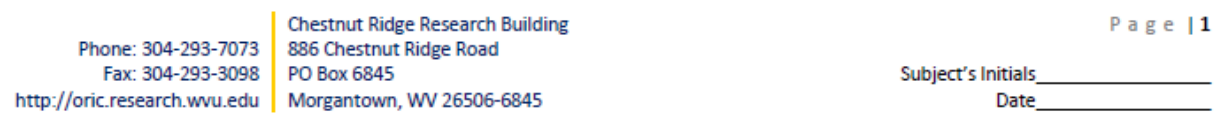


\section{W. WestVirginiaUniversity.}

Human Research Protocol

Assent Form

\section{Discomforts}

There are no known risks related to this study aside from any discomfort you may feel due to being observed.

\section{Benefits}

The benefits of this training are that your parent will learn how to help you develop your talker, participator, and problem solver repertoires.

\section{Confidentiality}

All data collected will be kept confidential. Only Liyu Chen will have direct access to it. The data will be stored securely. Videos will only be viewed by the research team for coding and interobserver agreement purposes and will be destroye once all presentation and publication possibilities are exhausted. Your data will only be displayed in ways that will not allow you to be identified

\section{Voluntary Participation}

Your participation is completely voluntary. You do not have to do this. No one will be mad at you if you refuse to do this and you may quit at any time. You have been allowed to ask questions about the research, and all of your questions were answered. Your decision about participation will not affect your relationship with your Tucci Learning Solutions behavior team.

I willingly agree to be in this research.

\section{Signatures}

Signature of Subject

Printed Name Date Time

The minor has had the opportunity to have questions addressed. The minor willingly agrees to be in the study.

Signature of Investigator or Co-Investigator

\begin{tabular}{|c|c|c|c|c|}
\hline \multicolumn{2}{|l|}{ Printed Name } & \multirow[t]{2}{*}{ Date } & \multicolumn{2}{|l|}{ Time } \\
\hline & Chestnut Ridge Research Building & & & Page 12 \\
\hline $\begin{array}{r}\text { Phone: } 304-293-7073 \\
\text { Fax: } 304-293-3098\end{array}$ & $\begin{array}{l}886 \text { Chestnut Ridge Road } \\
\text { PO Box } 6845\end{array}$ & & Subject's Initials & \\
\hline http://oric.research.wvu.edu & Morgantown, WV 26506-6845 & & Date_ & \\
\hline
\end{tabular}


Appendix E

Treatment Integrity

Effective Oral Lecture Discussion Implementation Integrity Observation

Checklist A

Observer:

Date:

Note: If the step is not applicable, write N/A in the + column and do not include in the calculation of fidelity.

\begin{tabular}{|l|c|c|l|}
\hline+ & - & Step & Checklist I: Reinforcement \\
\hline & & 1 & Trainer presents the definition \\
\hline & & 2 & Trainer presents the parameters \\
\hline & & 3 & Trainer presents the importance of reinforcement \\
\hline & & 4 & Trainer presents the schedule of reinforcement \\
\hline & & 5 & Trainer discusses the implementation \\
\hline & & 6 & Trainer and parent decide a protocol \\
\hline
\end{tabular}

\begin{tabular}{|l|l|c|l|}
\hline+ & - & Step & Checklist II: Prompting \\
\hline & & 1 & Trainer presents the definition \\
\hline & & 2 & Trainer presents the types of prompting \\
\hline & & 3 & Trainer presents 3-step prompt procedure \\
\hline & & 4 & Trainer presents Errorless prompt \\
\hline & & 5 & Trainer discusses the implementation \\
\hline & & 6 & Trainer and parent decide a protocol \\
\hline
\end{tabular}

\begin{tabular}{|l|l|c|l|}
\hline+ & - & Step & Checklist III: Fading \\
\hline & & 1 & Trainer presents the definition \\
\hline & & 2 & Trainer presents the importance of fading \\
\hline & & 3 & Trainer reviews the hierarchy of prompt \\
\hline & & 4 & Trainer discusses the implementation \\
\hline & & 5 & Trainer and parent decide a protocol \\
\hline
\end{tabular}

Total Number of + in Reinforcement section $/ 6=$ $\%$ Total Effective Oral Lecture Fidelity of Reinforcement

Total Number of + in Prompting section $/ 6=$ of Prompting $\%$ Total Effective Oral Lecture Fidelity

Total Number of + in Fading section $/ 5=$ Fading $\%$ Total Effective Oral Lecture Fidelity of 
Effective Model-Lead-Test Implementation Integrity Observation Checklist B

Observer:

Date:

Note: If the step is not applicable, write N/A in the + column and do not include in the calculation of fidelity.

\begin{tabular}{|l|c|c|l|}
\hline+ & - & Step & Checklist I: Reinforcement \\
\hline & & 1 & Trainer demonstrates \\
\hline & & 2 & Trainer leads the parent to do with her \\
\hline & & 3 & Trainer tests the parent to do on her/his own \\
\hline
\end{tabular}

\begin{tabular}{|c|c|c|l|}
\hline+ & - & Step & Checklist II: Prompt \\
\hline & & 1 & Trainer demonstrates \\
\hline & & 2 & Trainer leads the parent to do with her \\
\hline & & 3 & Trainer tests the parent to do on her/his own \\
\hline
\end{tabular}

\begin{tabular}{|c|c|c|l|}
\hline+ & - & Step & Checklist III: Fading \\
\hline & & 1 & Trainer demonstrates \\
\hline & & 2 & Trainer leads the parent to do with her \\
\hline & & 3 & Trainer tests the parent to do on her/his own \\
\hline
\end{tabular}

Total Number of + in Reinforcement section $/ 3=$ Fidelity of Reinforcement $\%$ Total Effective Oral Lecture

Total Number of + in Prompting section $/ 3=$ Prompting \% Total Effective Oral Lecture Fidelity of

Total Number of + in Fading section $/ 3=$ Fading $\%$ Total Effective Oral Lecture Fidelity of 
Appendix F

Social Validity Questionnaire for Parents in MLT

Name:

Date:

\begin{tabular}{|c|c|c|c|c|c|}
\hline Question for Parent to Answer & 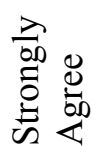 & 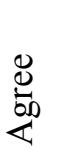 & $\begin{array}{l}\bar{\pi} \\
\stackrel{\Xi}{\Xi} \\
\vec{Z} \\
\bar{Z}\end{array}$ & 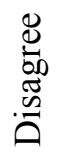 & 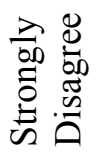 \\
\hline Appropriateness of Procedures & 5 & 4 & 3 & 2 & 1 \\
\hline $\begin{array}{l}\text { 1. The Model-Lead-Test procedure was } \\
\text { easy to read and understand }\end{array}$ & & & & & \\
\hline $\begin{array}{l}\text { 2. My trainer understood and } \\
\text { communicated procedures and } \\
\text { techniques effectively. }\end{array}$ & & & & & \\
\hline $\begin{array}{l}\text { 3. The interventions were easy to } \\
\text { implement. }\end{array}$ & & & & & \\
\hline Social Significance of Goals & 5 & 4 & 3 & 2 & 1 \\
\hline $\begin{array}{l}\text { 4. I would recommend a similar } \\
\text { intervention to other parents. }\end{array}$ & & & & & \\
\hline $\begin{array}{l}\text { 5. It is important to learn therapeutic } \\
\text { interventions to teach my child skills. }\end{array}$ & & & & & \\
\hline Social Importance of the Effects & 5 & 4 & 3 & 2 & 1 \\
\hline $\begin{array}{l}\text { 6. The skills learned by my child are } \\
\text { beneficial to their development. }\end{array}$ & & & & & \\
\hline $\begin{array}{l}\text { 7. I will continue to use these } \\
\text { interventions with my child when a new } \\
\text { skill needs to be taught. }\end{array}$ & & & & & \\
\hline $\begin{array}{l}\text { 8. My child learned the skills } \\
\text { effectively. }\end{array}$ & & & & & \\
\hline
\end{tabular}


Appendix G

Oral Lecture Discussion Training

Lesson I Handout (Reinforcement)

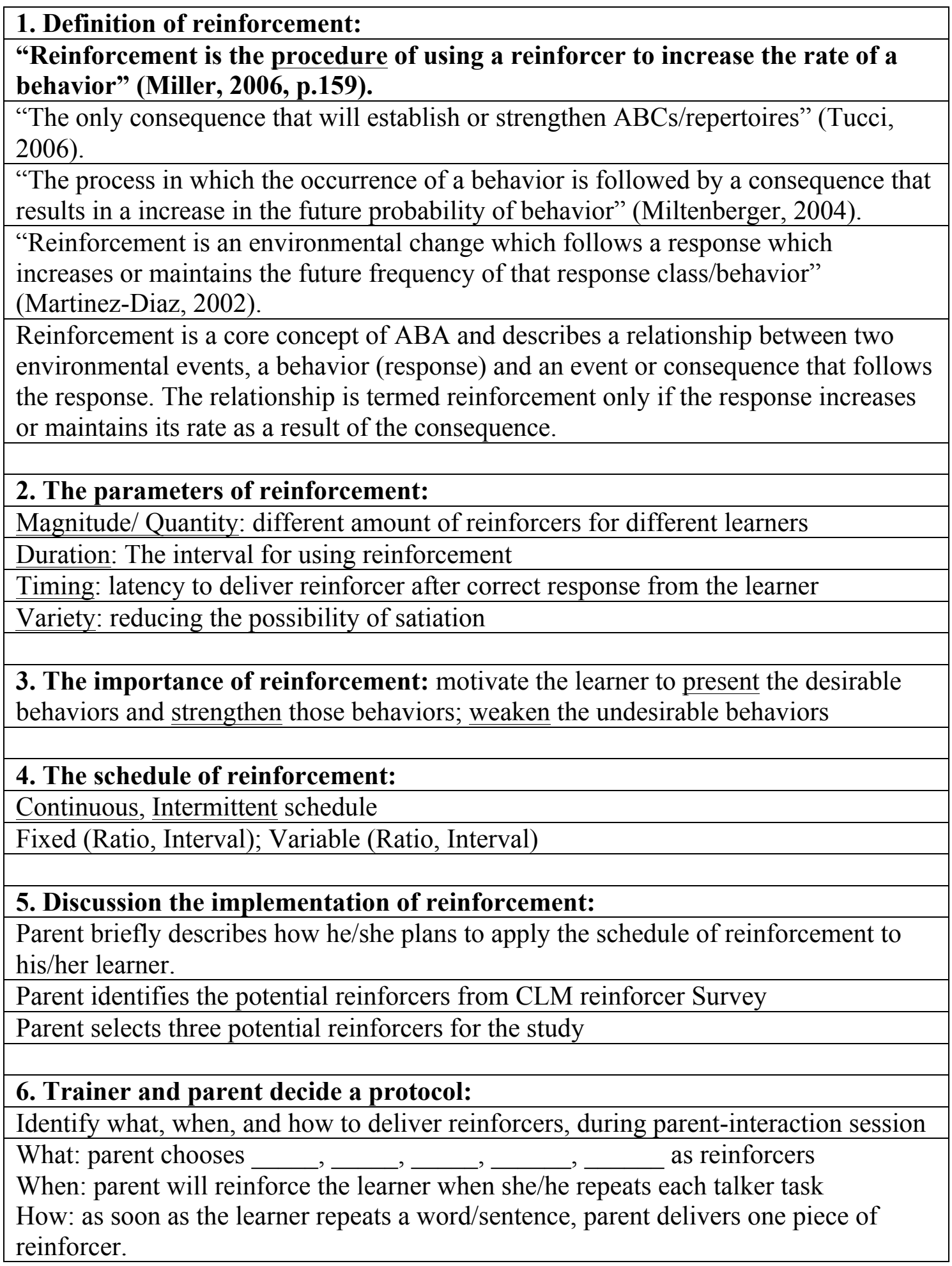




\author{
Appendix $\mathrm{H}$ \\ Oral Lecture Training \\ Lesson II Handout (Prompting)
}

\begin{abstract}
1. Definition of prompting:
"A prompt is an added stimulus that increases the probability that a person will make the correct response in the presence of a novel stimulus. It is usually withdrawn as soon as is practical" (Miller, 2006, p.339).

"A prompt is used to increase the likelihood that a person will evoke in the correct behavior at the correct time" (Miltenberger, 2004, p.195).

A prompt is an additional stimulus that increases the probability that a response will occur when a discriminative stimulus has been presented and has failed (Alberto, \& Troutman, 2013, p.298).
\end{abstract}

\title{
2. The types of prompting:
}

Full/partial physical guidance, Full/partial modeling, Full/partial verbal prompt, Gesture, Positional, Point, Environmental

Most to least prompt: Using when teaching a new skill, and then systematically fading down to least intrusive prompt

Least to most prompt: Using when a learner has learned the skill and is working towards mastery and independent level.

\section{Three-step prompt procedure:}

Step 1, Initial Instruction; Step 2, Instruction + Gesture/Model; Step 3, Instruction + Physical Guidance

\section{Errorless prompt:}

The prompt will be provided within 3 seconds when the target behavior is not present. The 4-step error correction procedure, model-prompt-switch-repeat, will be provided as needed.

\section{Discussion the implementation of prompting:}

Parent reviews his/her regular habits applying the different prompting to the learner Parent identifies 3 target behaviors of child with new prompting techniques Parent describes or demonstrates most to least prompt to a learner behavior Parent describes or demonstrates least to most prompt to a learner behavior

\section{Parent and trainer decide a protocol for what, when and how to deliver prompting for CLM participator repertoire of the learner}

What: identify the behavior we are going to implement prompt with, what activity, and what prompts we plan to apply (ex. Participating reading a story; Verbal, gesture and physical prompts will be apply)

When: identify the time period the parent would like to implement (ex. 5:30 pm)

How: identify how to direct the learner to participate, how to implement prompts if the learner doesn't respond right, how to reinforce the learner if he does correctly (ex. Say, Jack, let's read together; if the learner comes to table immediately, Jelly bean will be provide. If not, verbal prompt-John, come to table to read. If not, gesture, point to table. If not, physically guiding John to the able) 
THE IMPACT OF MODEL-LEAD-TEST ON PARENTS

101 


\author{
Appendix I \\ Oral Lecture Training \\ Lesson III Handout (fading)
}

\begin{tabular}{|c|}
\hline $\begin{array}{l}\text { 1. Definition of fading: } \\
\text { "Fading is the temporary use a prompt to establish a specific discrimination. } \\
\text { Prompt is gradually withdrawn as soon as is practical" (Miller, 2006, p.340). }\end{array}$ \\
\hline $\begin{array}{l}\text { Progressively providing less intrusive prompts and eventually no prompt for an } \\
\text { individual when a new skill has been learned with a particular prompt level. }\end{array}$ \\
\hline 2. The importance of fading: \\
\hline $\begin{array}{l}\text { Encouraging an individual to be more independent when learning a new behavior or } \\
\text { skill in order to avoid prompt dependency. }\end{array}$ \\
\hline 3. Reviewing the types of prompting: \\
\hline $\begin{array}{l}\text { Full/partial physical guidance, Full/partial modeling, Full/partial verbal prompt, } \\
\text { Gesture, Positional, Point, Environmental }\end{array}$ \\
\hline 4. Most to least prompting: \\
\hline $\begin{array}{l}\text { Full/partial physical guidance, Full/partial modeling, Gesture, Full/partial verbal } \\
\text { prompt, Independent }\end{array}$ \\
\hline 5. Discussion the implementation of fading: \\
\hline Parent reviews his/her regular fading habits (3- 5 examples) \\
\hline Parent verbally describes most to least prompt to a learner behavior \\
\hline Parent identifies 3 target behaviors of child with reliable new fading techniques \\
\hline $\begin{array}{l}\text { 6. Trainer and parent decide a protocol for what, when and how to deliver } \\
\text { fading for CLM participator repertoire of the learner: }\end{array}$ \\
\hline $\begin{array}{l}\text { What: identify what behavior will be worked on (ex. Brushing teeth), checking the } \\
\text { current prompts } \\
\text { When: ex. } 8 \text { pm } \\
\text { How: ex. When the learner starts brushing teeth, the parent will only do partial } \\
\text { physical prompt }\end{array}$ \\
\hline
\end{tabular}


Appendix $\mathrm{J}$

Accuracy of Oral Lecture Discussion Implementation for Parent Participant Checklist I (Reinforcement)

Observer:

Date:

Note: If participant answers researcher's question independently, mark Yes, independently. If the participant fails to independently respond either more than 3 consecutive questions or all of the answers are less than $80 \%$ accuracy, then the researcher will review the contents with which the parent has made errors with the parent participant. The researcher will only provide two verbal prompts to the parent participant when she/he doesn't respond independently. After the parent still doesn't respond correct, then "No" will be marked.

Participant will only need to fill blanks for the question that researcher asks.

\begin{tabular}{|c|c|c|}
\hline \multicolumn{3}{|c|}{ 1. Definition of reinforcement: } \\
\hline \multicolumn{3}{|c|}{$\begin{array}{l}\text { "Reinforcement is the procedure of using a reinforcer to increase the rate of a } \\
\text { behavior" (Miller, 2006, p.159). }\end{array}$} \\
\hline \multicolumn{3}{|c|}{ Q1. Does the parent answer the concepts above? } \\
\hline Yes, Independently & Yes with prompt 1,2 & No \\
\hline \multicolumn{3}{|c|}{ 2. The parameters of reinforcement: } \\
\hline \multicolumn{3}{|c|}{ Magnitude/ Quantity; Duration; Timing; Variety } \\
\hline \multicolumn{3}{|c|}{ Q2. Does the parent answer all four parameters above? } \\
\hline Yes, Independently & Yes with prompt 1,2 & No \\
\hline \multicolumn{3}{|c|}{ 3. The importance of reinforcement: } \\
\hline \multicolumn{3}{|c|}{$\begin{array}{l}\text { Reinforcement can help a learner stay motivated and help a learner establish, and } \\
\text { strengthen desirable behaviors and weaken undesirable behavior. }\end{array}$} \\
\hline \multicolumn{3}{|c|}{ Q3. Does the parent answer the importance of reinforcement? } \\
\hline Yes, Independently & Yes with prompt 1,2 & No \\
\hline \multicolumn{3}{|c|}{ 4. The schedule of reinforcement: } \\
\hline \multicolumn{3}{|c|}{ Continuous, Intermittent schedule } \\
\hline \multicolumn{3}{|c|}{ Fixed (Ratio, Interval); Variable (Ratio, Interval) } \\
\hline \multicolumn{3}{|c|}{ Q4. Does the parent answer all of the schedules of reinforcement above? } \\
\hline Yes, Independent & Yes with prompt 1,2 & No \\
\hline \multicolumn{3}{|c|}{ 5. Discussion the implementation of reinforcement: } \\
\hline \multicolumn{3}{|c|}{ Parent briefly describes how to apply the schedule of reinforcement to the learner. } \\
\hline \multicolumn{3}{|c|}{ Parent identifies the potential reinforcers from Tucci reinforcer Survey } \\
\hline \multicolumn{3}{|c|}{ Parent selects three potential reinforcers for the study } \\
\hline \multicolumn{3}{|c|}{ Q5. Does the parent decide the schedule of reinforcers? } \\
\hline Yes, independently & Yes with prompt 1,2 & No \\
\hline \multicolumn{3}{|c|}{ Q5.1. Does the parent complete the potential reinforcer survey? } \\
\hline Yes, independently & Yes with prompt 1,2 & No \\
\hline \multicolumn{3}{|c|}{ Q5.2. Does the parent select three potential reinforcers? } \\
\hline Yes, independently & Yes with prompt 1,2 & No \\
\hline
\end{tabular}




\section{THE IMPACT OF MODEL-LEAD-TEST ON PARENTS}

104

Q6. Does the parent decide the protocol for when, what, and how to deliver reinforcers during the study?

Yes, independently

Yes with prompt 1,2

No 


\section{Appendix K}

Accuracy of Oral Lecture Discussion Implementation for Parent Participant Checklist II (Prompting)

Observer:

Date:

\section{Definition of prompting:}

"A prompt is an added stimulus that increases the probability that a person will make the correct response in the presence of a novel stimulus. It is usually withdrawn as soon as is practical" (Miller, 2006, p.339).

Q1. Does the parent answer any of the concepts above?

\begin{tabular}{|l|l|l} 
Yes, independently & Yes with prompt 1,2 & No
\end{tabular}

\section{The types of prompting:}

Full/partial physical guidance, Full/partial modeling, Full/partial verbal prompt, Gesture, Positional, Point, Environmental

Most to least prompt: Using when teaching a new skill, and then systematically fading down to least intrusive prompt

Least to most prompt: Using when a learner has learned the skill and is working towards mastery and independent level.

Q2. Does the parent answer the types of prompting?

\begin{tabular}{|l|l|l} 
Yes, independently & Yes with prompt 1,2 & No
\end{tabular}

\section{Three-step prompt procedure:}

Step 1, Initial Instruction; Step 2, Instruction + Gesture/Model; Step 3, Instruction + Physical Guidance

Q3. Does the parent answer the three-step prompt procedure?

\begin{tabular}{|l|l|l} 
Yes, independently & Yes with prompt 1,2 & No
\end{tabular}

\section{Errorless prompt:}

The prompt will be provided within 3 seconds when the target behavior is not present. The 4-step error correction procedure, model-prompt-switch-repeat, will be provided as needed.

Q4. Does the parent answer the concept of errorless prompt?

\begin{tabular}{l|l|l} 
Yes, independently & Yes with prompt 1,2 & No
\end{tabular}

\section{Discussion the implementation of prompting:}

Parent reviews his/her regular habits applying the different prompting to the learner Q5. Does the parent review his/her regular habits applying the different prompting to the learner?

\begin{tabular}{l|l|l} 
Yes, independently & Yes with prompt 1,2 & No
\end{tabular}

Parent identifies 3 target behaviors of child with new prompting techniques

Q5.1. Does the parent identify 3 target behaviors of child with new prompting techniques?

\begin{tabular}{l|l|l} 
Yes, independently & Yes with prompt 1,2 & No
\end{tabular}

Parent describes or demonstrates most to least prompt to a learner behavior

Q5.2. Does the parent describe or demonstrate most to least prompt to a learner behavior?

\begin{tabular}{|l|l|l} 
Yes, independently & Yes with prompt 1,2 & No
\end{tabular}


106

Parent describes or demonstrates least to most prompt to a learner behavior

Q5.3. Does the parent describe or demonstrate least to most prompt to a learner behavior?

Yes, independently

Yes with prompt 1,2

No

6. Parent and trainer decide a protocol for when, what, and how to deliver prompting for CLM participator repertoire of the learner

Q6. Does the parent decide the protocol for when, what, and how to deliver prompting during the study?

Yes, independently

Yes with prompt 1,2

No 
Appendix L

Accuracy of Oral Lecture Discussion Implementation for Parent Participant Checklist III (Fading)

Observer:

Date:

\section{Definition of fading:}

"Fading is the temporary use a prompt to establish a specific discrimination. Prompt is gradually withdrawn as soon as is practical" (Miller, 2006, p.340).

Q1. Does the parent define the concept of fading?

\begin{tabular}{|l|l|l} 
Yes, independently & Yes with prompt 1,2 & No
\end{tabular}

\section{The importance of fading:}

Encouraging an individual to be more independent when learning a new behavior or skill in order to avoid prompt dependency.

Q2. Does the parent describe the importance of fading?

\begin{tabular}{|l|l|l} 
Yes, independently & Yes with prompt 1,2 & No
\end{tabular}

\section{Reviewing the types of prompting:}

Full/partial physical guidance, Full/partial modeling, Full/partial verbal prompt, Gesture, Positional, Point, Environmental

Q3. Does the parent review the types of the prompt?

\begin{tabular}{l|l} 
Yes, independently & Yes with prompt 1,2
\end{tabular}

No

\section{Most to least prompting:}

Full/partial physical guidance, Full/partial modeling, Gesture, Full/partial verbal prompt, Independent

Q3. Does the parent describe or demonstrate most to least prompting?

\begin{tabular}{|l|l|l} 
Yes, independently & Yes with prompt 1,2 & No
\end{tabular}

\section{Discussion the implementation of fading:}

Parent reviews his/her regular fading habits (3- 5 examples)

Q5. Does the parent review his/her regular fading habits?

\begin{tabular}{|l|l|l}
\hline Yes, independently & Yes with prompt 1,2 & No
\end{tabular}

Parent verbally describes most to least prompt to a learner behavior

Q5.1. Does the parent verbally describe most to least prompt to a learner behavior?

\begin{tabular}{l|l|l} 
Yes, independently & Yes with prompt 1,2 & No
\end{tabular}

Parent identifies 3 target behaviors of child with reliable new fading techniques

Q5.2. Does the parent identifies 3-5 target behaviors of child with reliable new fading techniques?

\begin{tabular}{|l|l|l} 
Yes, independently & Yes with prompt 1,2 & No
\end{tabular}

\section{Trainer and parent decide a protocol for when, what, and how to deliver} fading for CLM problem solver repertoire of the learner:

Q6. Does the parent decide a protocol for when, what, and how to deliver fading for

CLM problem solver repertoire of the learner?

\begin{tabular}{|l|l|l}
\hline Yes, independently & Yes with prompt 1,2 & No
\end{tabular}


108

Appendix M

Oral Lecture Discussion Training Interobserver Agreement Checklist I (Reinforcement)

Observer:

Date:

Note: If the step is not applicable, write N/A in the column and do not include in the calculation.

$(+)=$ The parent answers the question independently, as long as the parent answers correctly for the blanks in the question

$(+/ p)=$ the parent answers the question with prompts and less than two prompts; mark 1 when it is the first prompt and 2 for the second prompt

$(-)=$ The parent answers the question more than two prompts or the answer doesn't relate to any key words

Opportunity: opp

\begin{tabular}{|c|c|c|c|l|}
\hline+ & $+/ \mathrm{p}$ & - & opp & Checklist I: Effective of Reinforcement implementation \\
\hline & & 1 & $\begin{array}{l}\text { 1. When the researcher asks, "please describe the definition } \\
\text { of reinforcement", does parent answer any questions } \\
\text { regarding to the definition? }\end{array}$ \\
\hline & & 2 & $\begin{array}{l}\text { 2. When the researcher asks, "Please describe the parameters } \\
\text { of reinforcement", does parent answer any questions } \\
\text { regarding to the parameters? }\end{array}$ \\
\hline & 3 & $\begin{array}{l}\text { 3. When the researcher asks, "Please describe the importance } \\
\text { of reinforcement", does parent answer any questions } \\
\text { regarding to the importance of reinforcement? }\end{array}$ \\
\hline & 4 & $\begin{array}{l}\text { 4. When the researcher asks, "Please describe the schedule of } \\
\text { reinforcement", does parent answer any questions regarding } \\
\text { to the schedule of reinforcement? }\end{array}$ \\
\hline & 5 & $\begin{array}{l}\text { 5. When the researcher asks, "Please give me 3 examples for } \\
\text { how you usually apply reinforcers to your learner", does } \\
\text { parent review 3 examples of his/her regular habits applying } \\
\text { reinforcers? }\end{array}$ \\
\hline & 6 & $\begin{array}{l}\text { 5.1 When the researcher asks, "Let's check the result of the } \\
\text { reinforcer survey", does parent completes the potential } \\
\text { reinforcer survey? }\end{array}$ \\
\hline 7 & $\begin{array}{l}\text { 5.2 When the researcher asks, "Please show me the three } \\
\text { potential reinforcers you would like to use for this study", } \\
\text { does parent selects three potential reinforcers }\end{array}$ \\
\hline & 8 & $\begin{array}{l}\text { 5.3 When the researcher asks, "What is the ratio or interval } \\
\text { you decide to design for this study, does parent decide the } \\
\text { schedule of reinforcers }\end{array}$ \\
\hline $\begin{array}{l}\text { 6. When the researcher asks, "Let's decide the protocol for } \\
\text { when, what, and how to deliver reinforcer in this study, does } \\
\text { parent decide the protocol? }\end{array}$ \\
\hline
\end{tabular}


Appendix N

Oral Lecture Discussion Training Interobserver Agreement Checklist II (Prompting)

Observer:

Date:

\begin{tabular}{|c|c|c|c|c|}
\hline+ & $+/ \mathrm{p}$ & - & opp & Checklist II: Effective of prompting implementation \\
\hline & & & 1 & $\begin{array}{l}\text { 1. When the researcher asks, "Please describe the definition } \\
\text { of prompting", does parent answer any questions regarding to } \\
\text { the definition? }\end{array}$ \\
\hline & & & 2 & $\begin{array}{l}\text { 2. When the researcher asks, "Please describe the types of } \\
\text { prompting, does parent answer any questions regarding to the } \\
\text { types of prompting? }\end{array}$ \\
\hline & & & 3 & $\begin{array}{l}\text { 3. When the researcher asks, "Please describe the three-step } \\
\text { prompt procedure, does parent answer any questions } \\
\text { regarding to the procedure? }\end{array}$ \\
\hline & & & 4 & $\begin{array}{l}\text { 4. When the researcher asks, "Please describe the errorless } \\
\text { prompt", does parent answers any questions regarding to the } \\
\text { errorless prompt? }\end{array}$ \\
\hline & & & 5 & $\begin{array}{l}\text { 5. When the researcher asks, Please give me } 3 \text { examples for } \\
\text { how you usually provide prompting to your learner, does } \\
\text { parent review his/her regular prompting habits and give } 3 \\
\text { examples? }\end{array}$ \\
\hline & & & 6 & $\begin{array}{l}\text { 5.1 When the researcher asks, "Please describe how you are } \\
\text { going to apply new prompting techniques to } 3 \text { target } \\
\text { behaviors", does parent identify } 3 \text { target behaviors of child } \\
\text { with new prompting techniques }\end{array}$ \\
\hline & & & 7 & $\begin{array}{l}\text { 5.2 When the researcher asks, "Please describe how to apply } \\
\text { most to least prompt to learner's } 1 \text { target behavior ", does } \\
\text { parent verbally describe it? }\end{array}$ \\
\hline & & & 8 & $\begin{array}{l}5.3 \text { When the researcher asks, "Please describe how to apply } \\
\text { least to most prompt to learner's } 1 \text { target behavior", does } \\
\text { parent verbally describe it? }\end{array}$ \\
\hline & & & 9 & $\begin{array}{l}\text { 6. When the researcher asks, "Please describe when, what, } \\
\text { and how to deliver prompting in this study", does parent } \\
\text { decide the protocol? }\end{array}$ \\
\hline
\end{tabular}


Appendix $\mathrm{O}$

Oral Lecture Discussion Training Interobserver Agreement Checklist III (Fading)

Observer:

Date:

\begin{tabular}{|c|c|c|c|c|}
\hline+ & $+/ \mathrm{p}$ & - & opp & Checklist III: Effective of fading implementation \\
\hline & & & 1 & $\begin{array}{l}\text { 1. When the researcher asks, "Please describe the definition } \\
\text { of fading", does parent answers any questions regarding to } \\
\text { the definition? }\end{array}$ \\
\hline & & & 2 & $\begin{array}{l}\text { 2. When the researcher asks, "Please describe the importance } \\
\text { of fading", does parent answers any questions regarding to } \\
\text { the importance of fading }\end{array}$ \\
\hline & & & 3 & $\begin{array}{l}\text { 3. When the researcher asks, "Please describe the types of } \\
\text { prompting, does parent answer any questions regarding to the } \\
\text { types of prompting? }\end{array}$ \\
\hline & & & 4 & $\begin{array}{l}\text { 4. When the researcher asks, "Please describe the most to } \\
\text { least prompting", does parent answer it? }\end{array}$ \\
\hline & & & 5 & $\begin{array}{l}\text { 5. When the researcher asks, "Please describe how you } \\
\text { usually deliver the fading techniques with your learner's } \\
\text { three behaviors, does parent reviews his/her regular fading } \\
\text { habits }\end{array}$ \\
\hline & & & 6 & $\begin{array}{l}\text { 5.1 When the researcher asks, "Please describe how you } \\
\text { apply most to least prompt technique to your learner", does } \\
\text { parent verbally describe it? }\end{array}$ \\
\hline & & & 7 & $\begin{array}{l}5.2 \text { When the researcher ask, "Please identifies } 3 \text { target } \\
\text { behaviors of child with reliable new fading techniques", does } \\
\text { parent answer it? }\end{array}$ \\
\hline & & & 8 & $\begin{array}{l}\text { 6. When the researcher asks, "Let's decides the protocol for } \\
\text { when, what, and how to deliver fading for this study", does } \\
\text { parent answer it? }\end{array}$ \\
\hline
\end{tabular}

Total Number of agreement/total opportunity $=$ $\%$ interobserver agreement 
111

Appendix $\mathrm{P}$

Data Collection for Baseline, Oral Lecture Discussion training, MLT

Checklist I (Reinforcement)

Observer:

Date:

Directions: Watch the video segments (about 15 minute intervals in length). Observers will record "asking" and "reinforcing" behaviors of the parent participant. Parent participant follows protocol to ask the learner to repeat or answer sentences. For example, CLM Lesson 11.2 Repertoire, Talker 0.001 (mand >echoic): Repeating or T actions (nouns, verbs, and attributes) without item displayed.

Observers will circle one opportunity when every time the parent participant "asks" the learner to repeat/answer sentences.

Mark + if the learner repeats sentences or answers questions.

Mark + if parent participant provides "reinforcers" to the learner for repeating sentences or answering questions correctly.

\begin{tabular}{|c|l|l|}
\hline Opportunity & Learner Response & Parent Response \\
\hline 1 & & \\
\hline 2 & & \\
\hline 3 & & \\
\hline 4 & & \\
\hline 5 & & \\
\hline 6 & & \\
\hline 7 & & \\
\hline 8 & & \\
\hline 9 & & \\
\hline 10 & & \\
\hline 11 & & \\
\hline 12 & & \\
\hline & & \\
\hline & & \\
\hline & & \\
\hline & & \\
\hline
\end{tabular}

Definition of asking: The parent participant asks the learner to repeat the prewritten sentence scripts. (Opportunity)

Definition of reinforcing: The parent participant delivers reinforcers within 3 seconds with specific praise identifying desirable behaviors when the learner repeats the sentence scripts. (Parent response)

Definition of repeating: The learner attempts to repeat parent's questions within 3 seconds. (Learner response)

Definition of answering: The learner attempts to answer parent's questions within 3 seconds. (Learner response) 
Appendix Q

Data Collection for Baseline, Oral Lecture Discussion, and MLT Checklist II (Prompting)

Observer:

Date:

Directions: Watch the video segments (about 15 minute intervals in length). Observers will record "asking" and "prompting" behaviors of the parent participant. Parent participant follows protocol to ask the learner to participate activities. For example, CLM Lesson 8 repertoire, 0.501 participator (teacher-directed): Performs 2 sets of five consecutive responses (i.e., motor, written, vocal/sign behaviors).

Observers will circle one opportunity when every time the parent participant "asks" the learner to participate activities and will note what kind of prompts parent provides when the learner fails to respond within 3 seconds. Only two prompts will be provided in the research design.

Mark + if the learner responds correctly after his/her parent provides two prompts based on the protocol.

Mark + if parent participant provides "prompts" to the learner for following direction. Mark $+/ \mathrm{v}$ for parent provides verbal prompt; Mark +/vg for parent provides verbal and gesture prompt; Mark +/vp for parent provides verbal and physical prompt.

\begin{tabular}{|c|c|c|}
\hline Opportunity & Learner Response & Parent Response \\
\hline 1 & & \\
\hline 2 & & \\
\hline 3 & & \\
\hline 4 & & \\
\hline 5 & & \\
\hline 6 & & \\
\hline 7 & & \\
\hline 8 & & \\
\hline 9 & & \\
\hline 10 & & \\
\hline 12 & & \\
\hline & & \\
\hline & & \\
\hline
\end{tabular}

Definition of asking: The parent participant asks the learner to participate activities. (Per opportunity)

Definition of prompting: The parent participant delivers prompts within 3 seconds with specific promptings. (Parent response)

Definition of getting: The learner attempts to participate parent's direction within 3 seconds. (Learner response) 
Appendix R

Data Collection for Baseline, Oral Lecture Discussion, and MLT

Checklist III (Fading)

Observer:

Date:

Directions: Watch the video segments (about 15 minute intervals in length). Observers will record "fading" behaviors of the parent participant. Parent participant follows protocol to fade prompts for the skills that the learner gradually shows mastery. For example, CLM Lesson 12 Repertoire, Problem solver (operate): Solves problem by manipulating a variety of different size and shape parts to fit them in place or remove them from a place (e.g., twists off a cap); completes 2 sets of five pieces of each (up to 10 parts all together).

Observers will circle one opportunity when every time the parent participant attempts to prompt the learner.

Mark + if the learner does each action independently.

Mark $+/$ fp if the parent provides full physical prompt, mark $+/$ pp for partial physical prompt, mark $+/$ m for model prompt, mark $+/$ g for gesture prompt, mark $+/ \mathrm{v}$ for verbal prompt

\begin{tabular}{|c|l|l|}
\hline Opportunity & Learner Response & Parent Response/Fading \\
\hline 1 & & \\
\hline 2 & & \\
\hline 3 & & \\
\hline 4 & & \\
\hline 5 & & \\
\hline 6 & & \\
\hline 7 & & \\
\hline 8 & & \\
\hline 9 & & \\
\hline 10 & & \\
\hline 11 & & \\
\hline 12 & & \\
\hline & & \\
\hline & & \\
\hline
\end{tabular}

Definition of fading: Fading the full verbal/model/physical prompt by gradually reducing the model then the physical guidance to partial verbal prompt

Definition of responding: The learner attempts to manipulate parts to solve problem. 


\section{Appendix S}

Accuracy of MLT Implementation for Parent Participant Checklist I (Reinforcement)

Observer:

Date:

Note: If the step is not applicable, write N/A in the + column and do not include in the calculation of fidelity.

Opportunity: opp

Mark + if yes; Mark +/p 1 if yes with the first prompt; Mark +/p 2 if yes with the second prompt; Mark - if the parent doesn't respond correctly.

\begin{tabular}{|c|c|c|c|c|}
\hline+ & $+/ \mathrm{p}$ & - & opp & Checklist I: Reinforcement \\
\hline & & & 1 & \multirow{5}{*}{$\begin{array}{l}\text { After the trainer demonstrates how to } \\
\text { implement the reinforcer, does the } \\
\text { parent deliver the reinforcer with the } \\
\text { trainer based on the protocol to the } \\
\text { learner? }\end{array}$} \\
\hline & & & 2 & \\
\hline & & & 3 & \\
\hline & & & 4 & \\
\hline & & & 5 & \\
\hline & & & 1 & \multirow{5}{*}{$\begin{array}{l}\text { After the trainer demonstrates and leads } \\
\text { the parent how to implement the } \\
\text { reinforcer, does the parent deliver the } \\
\text { reinforcer on her/his own based on the } \\
\text { protocol to the learner? }\end{array}$} \\
\hline & & & 2 & \\
\hline & & & 3 & \\
\hline & & & 4 & \\
\hline & & & 5 & \\
\hline
\end{tabular}




\section{Appendix T}

Accuracy of MLT Implementation for Parent Participant Checklist II (Prompting)

Observer:

Date:

Note: If the step is not applicable, write N/A in the + column and do not include in the calculation of fidelity.

Opportunity: Opp

Mark + if yes; Mark +/p 1 if yes with the first prompt; Mark +/p 2 if yes with the second prompt; Mark - if the parent doesn't respond correctly.

\begin{tabular}{|c|c|c|c|c|}
\hline+ & $+/ \mathrm{p}$ & - & Opp & Checklist II: Prompt \\
\hline & & & 1 & \multirow{5}{*}{$\begin{array}{l}\text { After the trainer demonstrates how to } \\
\text { implement the three-step prompting, } \\
\text { does the parent deliver the prompting } \\
\text { with the trainer based on the protocol to } \\
\text { the learner? }\end{array}$} \\
\hline & & & 2 & \\
\hline & & & 3 & \\
\hline & & & 4 & \\
\hline & & & 5 & \\
\hline & & & 1 & \multirow{5}{*}{$\begin{array}{l}\text { After the trainer demonstrates and leads } \\
\text { the parent how to implement the three- } \\
\text { step prompting, does the parent deliver } \\
\text { the prompting on her/his own based on } \\
\text { the protocol to the learner? }\end{array}$} \\
\hline & & & 2 & \\
\hline & & & 3 & \\
\hline & & & 4 & \\
\hline & & & 5 & \\
\hline
\end{tabular}


Appendix U

Accuracy of MLT Implementation for Parent Participant Checklist III (Fading)

Observer:

Date:

Note: If the step is not applicable, write N/A in the + column and do not include in the calculation of fidelity.

Opportunity: Opp

Mark + if yes; Mark +/p 1 if yes with the first prompt; Mark +/p 2 if yes with the second prompt; Mark - if the parent doesn't respond correctly.

\begin{tabular}{|c|c|c|c|c|}
\hline+ & $+/ \mathrm{p}$ & - & opp & Checklist III: Fading \\
\hline & & & 1 & \multirow{5}{*}{$\begin{array}{l}\text { After the trainer demonstrates how to } \\
\text { implement the fading procedure, does } \\
\text { the parent deliver the fading with the } \\
\text { trainer based on the protocol to the } \\
\text { learner? }\end{array}$} \\
\hline & & & 2 & \\
\hline & & & 3 & \\
\hline & & & 4 & \\
\hline & & & 5 & \\
\hline & & & 1 & \multirow{5}{*}{$\begin{array}{l}\text { After the trainer demonstrates and leads } \\
\text { the parent how to implement the fading } \\
\text { procedure, does the parent deliver the } \\
\text { fading on her/his own based on the } \\
\text { protocol to the learner? }\end{array}$} \\
\hline & & & 2 & \\
\hline & & & 3 & \\
\hline & & & 4 & \\
\hline & & & 5 & \\
\hline
\end{tabular}

